\title{
The Composition of Cigarette Smoke: A Catalogue of the Polycyclic Aromatic Hydrocarbons*
}

\author{
by \\ Alan Rodgman ${ }^{1}$ and Thomas A. Perfetti ${ }^{2}$ \\ ${ }^{1} 2828$ Birchwood Drive, Winston-Salem, North Carolina, 27103-3410, USA \\ ${ }^{2}$ Perfetti and Perfetti, LLC, 2116 New Castle Drive, Winston-Salem, North Carolina, 27103-5750, USA
}

\section{SUMMARY}

Classified as toxicants in many of the substances to which humans are exposed are the polycyclic aromatic hydrocarbons (PAHs). Such exposures include air pollutants from a variety of sources, foodstuffs and beverages, and tobacco smoke. Since the early 1950 s, the composition of the latter has been more completely defined than that of any other consumer product. Nearly 4800 components have been identified in tobacco smoke and among these are over 500 PAHs either completely or partially identified. Because of the tumorigenicity of many PAHs, much research has been conducted in attempts to define the relationship between the PAH structures and their specific tumorigenicities in laboratory animals. None of the theories to date completely answers all the questions.

As a prelude to an attempt to develop a more reasonable $\mathrm{PAH}$ structure-tumorigenicity relationship, the PAHs completely or partially identified in cigarette smoke have been catalogued. In the catalogue, they are categorized as bicyclic, tricyclic, tetracyclic, etc. with each group subdivided into all-benzenoid PAHs and cyclopentanoid-benzenoid PAHs. Another tabulation includes the PAHs considered in several previous studies on structure-tumorigenicity relationships, studies that dealt primarily with allbenzenoid PAHs. [Beitr. Tabakforsch. Int. 22 (2006) 13-69]

\section{ZUSAMMENFASSUNG}

Mit toxisch eingestuften polycyclischen aromatischen Kohlenwasserstoffen (PAHs) sind Menschen vielfach exponiert. Die Expositionen umfassen Luftverschmutzung durch eine Vielzahl von Quellen, Lebensmittel, Getränke und Tabakrauch. Seit den frühen fünfziger Jahren des vorigen Jahrhunderts wurde die Zusammensetzung des letzteren umfassender untersucht als jedes andere Konsumgut. Fast 4800 Einzelsubstanzen wurden im Tabakrauch nachgewiesen und unter diesen sind mehr als 500 PAHs entweder vollständig oder teilweise identifiziert. Wegen der tumorigenen Wirkung vieler PAHs wurde in vielen Studien versucht, den Zusammenhang zwischen der Struktur der PAHs und ihrer spezifischen tumorigenen Wirkung bei Labortieren zu untersuchen. Durch keine dieser Theorien lassen sich bis heute alle Fragen vollständig beantworten. Als erster Schritt eines Versuchs, eine schlüssigere Beziehung zwischen der Struktur der PAHs und ihrer tumorigenen Wirkung zu entwickeln, wurden die PAHs, die vollständig oder teilweise im Zigarettenrauch identifiziert sind, katalogisiert. In dieser Systematisierung wird unterschieden zwischen bicyclischen, tricyclischen, tetracyclischen usw. PAHs, wobei wiederum jede Gruppe unterteilt ist in benzenoide und cyclopentanoide-benzenoide PAHs. Eine andere Einteilung bezieht sich auf diejenigen PAHs, die Gegenstand mehrerer früherer Studien waren, in denen der Zusammenhang zwischen Struktur und Tumorigenität, hauptsächlich benzenoider PAHs, untersucht wurde. [Beitr. Tabakforsch. Int. 22 (2006) 13-69]

\section{RESUME}

Les hydrocarbures polynucléaires aromatiques (PAHs), contenus dans de nombreuses substances auxquelles l'homme est exposé, sont classifiés comme toxiques. Parmi ces expositions comptent les polluants de l'air de sources multiples, l'alimentation, les boissons ou la fumée du tabac. Depuis le début des années 1950, la composition chimique de cette dernière a été analysée de façon plus approfondie que tout autre produit de consommation. Environ 4800 substances ont été identifiées dans la fumée du tabac et parmi celles-ci plus de 500 PAHs complètement ou partiellement identifiées. Due à la tumorigénicité de nombreux 
PAHs, beaucoup d'études ont été menées en vue d'examiner la relation entre les structures des PAHs et leurs tumorigénicités spécifiques chez les animaux de laboratoire. Jusqu'à présent aucune de ces théories n'explique complètement toutes les questions.

Une approche dans le but de développer une relation plus raisonnable entre la structure et la tumorigénicité des PAHs, est la classification des PAHs complètement ou partiellement identifiés. Dans ce catalogue, les PAHs sont classifiés comme bicycliques, tricycliques, tetracycliques, etc., chaque groupe étant subdivisé en PAHs benzenoid et cyclopentanoid-benzenoid. Un autre catalogue comprend les PAHs examinés dans des études antérieures sur la relation entre la structure et la tumorigénicité, surtout des PAHs benzenoid. [Beitr. Tabakforsch. Int. 22 (2006) 13-69]

\section{INTRODUCTION}

Tobacco and tobacco products in the forms of leaf, shredded or grounded tobacco, and various forms of cigars and cigarettes have been available to individuals for ages. For centuries people have enjoyed tobacco but have been admonished of its potential health concerns. Health concerns for cigarette smokers have increased steadily since the early 1950 s due to the rapid development and advancement in separation sciences, toxicology and medicine. In his 1954 publication, KOSAK (194) was the first person to catalogue compounds reported in tobacco smoke. His list contained fewer than 100 compounds and a significant number were incorrectly characterized. Today nearly 4800 compounds have been identified as components in tobacco smoke [see Figure 1, p. 140 in (125)]. Over the past fifty years, the Tobacco Industry has made significant progress in both the identification of tobacco and smoke components and the development of technologies to reduce cigarette smoke yields. Significant efforts continue in government, academia, and especially the Tobacco Industry to understand the health effects of smoking and to develop cigarette products with reduced health risks for smokers. One class of tobacco smoke components that has been studied extensively and intensively is the polycyclic aromatic hydrocarbons (PAH) due to their potential health concerns.

Periodically, tobacco researchers have reported the progress on the identification of tobacco and smoke components. Review articles by JOHNSTONE and PLIMMER (182) and IZAWA (177) detailed the tobacco and smoke research conducted over 100 years. IZAWA listed 440 identified smoke components by 1961. QUIN (289) published a review of components found in tobacco and smoke. HERRMANN (151) reviewed phenolic compounds in tobacco smoke. In 1963, PHILIP MORRIS (278) published a monograph on tobacco and smoke composition, a copy of which was provided the Advisory Committee on smoking and health to the US Surgeon General (445). In 1964, ELMENHORST and RECKZEH (97) tabulated the aromatic hydrocarbons identified in tobacco smoke. KUHN (202) published an article on alkaloids in tobacco and smoke. In their 1967 book, WYNDER and HOFFMANN (508) discussed tobacco and smoke chemistry and the results of animal studies with tobacco smoke. ELMENHORST and SCHULTZ (98) listed 250 low-boiling components and vapor-phase components identified in tobacco smoke. In his 1968 review, STEDMAN (425) listed nearly 1200 identified tobacco and smoke components. The next year, NEURATH (256) reported on the presence of 180 nitrogen-containing compounds in smoke. With the meaningful advancements in analytical methodology, the number of tobacco and smoke components increased dramatically (125). At R. J. Reynolds Tobacco Company (RJRT), SCHUMACHER et al. (391), HECKMAN and BEST (147), and NEWELL et al. (264) identified over 1500 compounds in the water-soluble and ether-soluble fractions of tobacco smoke. In 1977, SCHMELTZ and HoFFMANN (381) catalogued nearly $500 \mathrm{~N}$ containing compounds identified in tobacco smoke but their catalogue did not include the more than $230 \mathrm{~N}$-containing compounds newly identified in tobacco smoke by HECKMAN and BEST (147). Between 1974 and 1978, SNOOK et al. (416, 419-422) published the results of their massive study of the PAHs identified in tobacco smoke, a study that was followed by an equally definitive one published in 1981 on the azaarenes in tobacco smoke (418). In 1980, ISHIGURO and SUGAWARA (176) listed 1889 identified tobacco smoke components in their monograph. However, a tally of the reported tobacco smoke components at that time exceeded 2500. No additional catalogues of the total number of identified components of cigarette mainstream smoke (MSS) have been published since the 1980 ISHIGURO and SUGAWARA (176) publication. SMITH et al. (413) recently reported the chemical structures of the 253 identified phenols reported in cigarette MSS.

Numerous catalogues of PAHs identified in MSS have been compiled from 1955 through 2005, including this report. Table 1 is a chronology of catalogues of PAHs in MSS. It contains the year of each catalogue, author (and reference), and the number of PAHs listed. The previous catalogues contain much overlap in terms of the PAHs identified. This report attempts to eliminate the overlap and clearly present the 539 PAHs identified in MSS. Our present report is intended to present a referenced catalogue of the either completely or partially characterized ${ }^{\mathrm{a}} \mathrm{PAHs}$ in tobacco smoke. The catalogue contains the chemical name, structure, molecular weight, molecular formula, CAS registration number, and alphabetical listing of references on PAHs.

\section{THE IDENTIFICATION OF POLYCYCLIC AROMATIC HYDROCARBONS IN CIGARETTE MAINSTREAM SMOKE}

The significant increase in the number of studies on tobacco smoke composition was triggered by the following events: a) The results in the early 1950s from several retrospective epidemiology studies (487) in which it was reported that an association existed between cigarette smoking and the incidence of lung cancer in smokers, b) a 1953 report of the production of skin carcinoma in susceptible laboratory animals skin painted repeatedly with a concentrated solution of cigarette MSS condensate supposedly produced under conditions simulating the human

\footnotetext{
a The term "partially characterized" or "partially identified" indicates that the position of one or more alkyl substituents was not determined.
} 
Table 1. Chronology of catalogues of PAHs in MSS

\begin{tabular}{|c|c|c|c|}
\hline Year & Author & No. of PAHs listed & Ref. \\
\hline 1954 & Kosak & $4^{a}$ & 194 \\
\hline 1955 & Latimer & 10 & 211 \\
\hline 1957 & Latimer and Rodgman & 33 & 212 \\
\hline 1958 & Rodgman & 36 & 313 \\
\hline 1959 & Johnstone and Plimmer & 57 & 182 \\
\hline 1960 & Rodgman and Menz & 68 & 337 \\
\hline 1962 & Rodgman et al. & 77 & 339 \\
\hline 1963 & Philip Morris & 61 & 278 \\
\hline 1963 & Rodgman et al. & 77 & 340 \\
\hline 1964 & Elmenhorst and Reckzeh & 70 & 97 \\
\hline 1965 & Rodgman et al. & 85 & 338 \\
\hline 1967 & Rodgman and Woosley & 85 & 342 \\
\hline 1968 & Stedman & 79 & 425 \\
\hline 1975 & Roberts et al. & 206 & 303 \\
\hline 1976 & Snook et al. & $252^{b}$ & 421 \\
\hline 1977 & Snook et al. & $157^{b}$ & 419 \\
\hline 1978 & Snook et al. & $438^{b}$ & 420 \\
\hline 1980 & Ishiguro and Sugawara & 191 & 176 \\
\hline 1997 & Williams et al. & $427^{c}$ & 475 \\
\hline 2005 & Rodgman and Perfetti & $539^{d}$ & current \\
\hline
\end{tabular}

a Three of the PAHs listed were identified in a destructive distillate of tobacco, not in tobacco smoke.

${ }^{\mathrm{b}}$ In the three articles on the PAH study by SNOOK et al. (419421), some identified PAHs were listed in more than one article.

' In several instances, more than one isomer was reported for some monoalkyl-, dialkyl-, trialkyl-, and tetraalkyl-PAHs but the positions of the alkyl groups were not determined. In the case of such multiple alkyl isomers, only one was listed in this report.

${ }^{d}$ This list includes the number of isomers of monoalkyl-, dialkyl-, trialkyl-, and tetraalkyl-PAHs reported where the positions of the alkyl groups were not determined.

smoking of a cigarette (488), c) the realization in 1954 that very little (194) was known about the composition of tobacco smoke to which consumers had been exposed for nearly 400 years, and d) the incorporation of chromatography into the overall methodology of the fractionation of complex mixtures such as tobacco smoke.

Naturally, these findings raised several questions. The first dealt with the identity of the cigarette MSS component(s) responsible for the smoking-lung cancer association in smokers and the skin tumor induction in laboratory animals. Because of extensive data generated on the specific tumorigenicity of about $25 \%$ of the hundreds of PAHs synthesized between 1929 and the early 1950s (144), PAHs were considered the most likely tumorigenic agents in cigarette MSS even though their presence was not certain. Eventually, numerous PAHs were identified in cigarette MSS. Because of its MSS level and its high specific tumorigenicity in several bioassays, one PAH was subjected to intense scrutiny: Benzo $[a]$ pyrene $(\mathrm{B}[a] \mathrm{P})$. As a carcinogen, $\mathrm{B}[a] \mathrm{P}$ elicited carcinomas at the painting site in the mouse-skin bioassay. As a sarcogen, $\mathrm{B}[a] \mathrm{P}$ elicited sarcomas in rodent bioassays involving subcutaneous injection.

One class of tobacco smoke components studied extensively is the polycyclic aromatic hydrocarbons. As reported by RODGMAN (323), between 1950 and 1970, an extensive amount of research was conducted on tobacco- and cigarette smoke-related topics. The information generated led to the development of several significant cigarette design technologies that resulted in the modification of the delivery and composition of cigarette MSS.

The following is a brief chronology of the events occurring in the tobacco smoke-PAH situation: In 1939, the PAHs anthracene, phenanthrene, and $\mathrm{B}[a] \mathrm{P}$ were reported as components of a tobacco-related material by ROFFO (346-349) and his son (345). In discussions of tobacco smoke, the ROFFO findings are generally disregarded because the three PAHs they reported were not detected in tobacco smoke but in a destructive distillate of tobacco. However, ROFFO did report another finding that led to much research both within and outside the Tobacco Industry. ROFFO reported that comparison of the destructive distillate of tobacco with that of an ethanol-extracted tobacco indicated (350) that the PAH content and specific tumorigenicity of the extracted tobacco destructive distillate were reduced from those of the destructive distillate from the control tobacco. RoFFO speculated that the precursors of the tumorigenic PAH components of his distillates were ethanol-soluble phytosterols. Eventually his prediction, as far as it went, was found to be true for cigarette MSS $(327,398)$. Because he was unaware of the presence in tobacco of long-chained terpenoids such as solanesol, identified in flue-cured tobacco in 1957 by ROWLAND et al. (355), ROFFO obviously could not include them in his 1942 precursor prediction. It should be noted that the findings by ROFFO on destructive distillates of tobacco were subsequently equivalent to the effects observed in smoked tobacco, i.e., organic solvent-extraction of a tobacco or tobacco blend which was then incorporated into cigarettes gave MSS with reduced PAH levels and specific tumorigenicity to mouse skin compared to the MSS from control tobacco. However, usually the reduction in specific tumorigenicity was less than the reduction in PAHs, particularly $\mathrm{B}[a] \mathrm{P}$.

The generation in the early 1950 s of carcinomas in laboratory animals (mice) skin-painted with a solution of the mainstream 'tar' from commercial cigarettes (488) led to numerous studies to identify the possible causative agent(s) in the 'tar'. Since much more tumorigenicity data and knowledge were available on PAHs than on any other class of compounds, most of the effort was concentrated on identifying PAHs in cigarette smoke condensate (CSC) as the possible cause of the tumorigenicity. Because of its demonstrated potency as an initiator of carcinomas on skin painting and the wealth of information on it, $\mathrm{B}[a] \mathrm{P}$ became the target of much research on CSC. In 1951, HARTWELL (144) listed nearly 350 studies on the tumorigenicity of $\mathrm{B}[a] \mathrm{P}$ administered in various ways to various species. The other previously studied PAHs were dibenz $[a, h]$ anthracene (DB $[a, h] \mathrm{A})$ and 1,2-dihydro-3-methylbenz[j]aceanthrylene (3-methylcholanthrene) with 240 and 303 reported biological studies, respectively. Benz $[a]$ anthracene $(\mathrm{B}[a] \mathrm{A})$ and 7,12-dimethylbenz $[a]$ anthracene $(\mathrm{DMB}[a] \mathrm{A})$ were listed with 20 and 32 studies, respectively. In the 20 studies reported by HARTWELL (144), a malignant tumor was noted in only one instance with $\mathrm{B}[a] \mathrm{A}$.

Although $\mathrm{B}[a] \mathrm{P}$ was reported as a CSC component in the mid-1950s by several American (6, 46-48) and British investigators (69) on the basis of spectral evidence, FIESER, as late as 1957 (104), considered the published evidence to be inadequate as proof of the presence of $\mathrm{B}[a] \mathrm{P}$ in CSC. 
Obviously, in 1957 FIESER was unaware of the report by RODGMAN in 1956 (308) on the isolation of crystalline $\mathrm{B}[a] \mathrm{P}$ from MSS or the reports by FALK and KOTIN in 1955 and 1956 (100) on the determination of the per cigarette yields of $\mathrm{B}[a] \mathrm{P}$ (plus $\mathrm{B}[a] \mathrm{A}$ and dibenzo[def,p]chrysene) in MSS and sidestream smoke (SSS). Shortly thereafter, in 1959, WYNDER and HOFFMANN reported the isolation of $\mathrm{B}[a] \mathrm{P}$ in crystalline form from CSC (490), thus ending the controversy about its presence in cigarette smoke.

In 1954, knowledge of cigarette MSS composition was extremely limited. As mentioned earlier, KoSAK (194) listed fewer than 100 components reported in tobacco smoke and many of those listed were incorrect. Some of the early research on cigarette MSS composition, particularly the PAHs, was conducted at R. J. Reynolds Tobacco Company (RJRT) ${ }^{\mathrm{b}}$. Complete details of the experimental procedures and findings are available on the Internet at www.rjrtdocs.com.

The initial RJRT PAH investigation involved 11 PAHs in the MSS from non-filtered cigarettes $(308,312)$ [(see Table 1 in (323)]. Naphthalene, anthracene, pyrene, fluoranthene, and $\mathrm{B}[a] \mathrm{P}$, isolated in crystalline form, were characterized by UV absorption spectral data as well as by classical chemical means (mixture melting point, IR spectra, derivatization, and derivative properties). The other six PAHs were identified on the basis of agreement of their UV absorption spectra with those of authentic samples or with published UV data.

The second RJRT investigation involved the MSS from filter-tipped cigarettes $(315,329)$ [see Table 2 in $(323)^{\mathrm{c}}$ ]. In that study 43 PAHs, including the 11 PAHs found in the initial study were identified $(308,312)$. Of the 43 PAHs, 14 were isolated in crystalline form and characterized by both UV spectral and classical chemical means [see Table 1 in (323)]. $\mathrm{B}[a] \mathrm{P}, \mathrm{B}[a] \mathrm{A}, \mathrm{DB}[a, h] \mathrm{A}$, and several other PAHs were also isolated in crystalline form from the CSC $(308,312,329)$. The other 29 were identified from the agreement of their UV absorption spectra with those of authentic samples or with published spectra. $\mathrm{B}[a] \mathrm{P}, \mathrm{B}[a] \mathrm{A}$, and $\mathrm{DB}[a, h] \mathrm{A}$ had been reported to be tumorigenic to mouse skin although the bioassay data for $\mathrm{B}[a] \mathrm{A}$ were contradictory $(88,144)$.

Although much of the early research at RJRT R\&D on the identification of PAHs in MSS and the effect of various tobacco blends and/or treatments on their MSS yields was summarized in several recent publications $(323,341)$, other members of the US Tobacco Industry were also much

\footnotetext{
${ }^{b}$ Numerous formal in-house reports and memoranda authored by RJRT R\&D personnel are cited herein. Many have been published totally or in part in peer-reviewed journals and/or presented totally or in part at scientific conferences (Tobacco Chemists' Research Conferences, American Chemical Society Symposia on Tobacco and Smoke, CORESTA Conferences, etc.). Whether published, presented, or neither, copies of all RJRT reports cited are stored in various repositories such as the one in Minnesota. Their contents are available on the Internet address indicated. Experimental procedures used, data collected, and interpretations summarized here are described in detail in the reports cited.

${ }^{c}$ During the editing of the page proof for Reference 323, the author (A.R.) failed to notice the omission of an item from Table 2.
} Unfortunately, Item 38, chrysene was omitted. The line should read:

\begin{tabular}{l|c|c|c|c}
\hline No. & Polycyclic aromatic hydrocarbon & AC & PM & RJRT \\
\hline 38 & Chrysene & - & D & x
\end{tabular}

involved in similar research in the 1960s and 1970s. The following paragraphs provide a few examples of their early efforts:

At Philip Morris in 1963, RoBB et al. (301a) described the identification of 14 PAHs (naphthalene, fluorene, anthracene, 9-methylanthracene, phenanthrene, fluoranthene, pyrene, 1-methylpyrene, $\mathrm{B}[a] \mathrm{P}, \quad \mathrm{B}[e] \mathrm{P}), \quad \mathrm{DB}[a, h] \mathrm{A}$, benz $[e]$ acephenanthrylene, perylene, benzo[ghi]perylene), biphenyl, and the aza-arene, carbazole, in cigarette MSS. Almost all the details in this 1963 Philip Morris in-house report were subsequently presented at the 1964 meeting of the Cooperation Center for Scientific Research Relative to Tobacco (CORESTA) meeting and published in 1965 (302). Also at Philip Morris, CARPENTER (49a) in 1964 described the per cigarette $\mathrm{B}[a] \mathrm{P}$ yields from several commercial cigarettes; OAKLEY (265a) in 1965 reported the per cigarette $\mathrm{B}[a] \mathrm{P}$ yields from cigarettes fabricated from different tobacco types (flue-cured, burley, Oriental); SEGURA (395a) in 1966 reported the contribution of cigarette paper to the per cigarette $\mathrm{B}[a] \mathrm{P}$ yield; JOHNSON (180a) in 1965 described the effect of a tobacco additive, aluminum chloride, on the MSS B $[a] \mathrm{P}$ yield; and OAKLEY (265b) in 1966 determined the difference in per cigarette $\mathrm{B}[a] \mathrm{P}$ yield in MSS and SSS.

At British American Tobacco Company (BAT) in 1966, CHAKRABORTY and THORNTON (51a) studied the effect of various additives on MSS PAHs. The changes in the per cigarette yields of a variety of PAHs were determined. They included: anthracene, $\mathrm{B}[a] \mathrm{A}$, benzo[ $g h i]$ fluoranthene, benzo $[k]$ fluoranthene, $\mathrm{B}[a] \mathrm{P}, \mathrm{B}[e] \mathrm{P}$, chrysene, fluoranthene, fluorene, methylfluorene, phenanthrene, several alkylphenanthrenes, dimethylphenanthrene, pyrene, and several benzofluorenes. ${ }^{\mathrm{d}}$

Although studies on PAHs in MSS were conducted at RJRT and L\&M in the 1960s, publications only dealt with analytical techniques. For example, in 1963 MoLD et al. (239a) at Liggett and Myers Tobacco Company (L\&M) described the use of a compound, tetramethyluric acid, that complexes with polycyclic compounds. It was a procedure reminiscent of the finding of the water-soluble purine-PAH complex defined by WEIL-MALHERBE (470a), a finding subsequently developed into an alternative analytical method for the determination of PAHs and aza-arenes in tobacco smoke and other media by ROTHWELL and WHITEHEART (351-354). Although the study was not described as relating to tobacco smoke, CUNDIFF and MARKUNAS (72a) at RJRT in 1963 reported a titrimetric analysis of the nitro groups in numerous PAH:2,4,7-trinitrofluorenone complexes as a means to define the molecular weight of the PAH. All but one of the PAH:2,4,7-trinitrofluorenone complexes could be obtained from the PAH fraction of cigarette MSS. Of course, there were also methods developed for the in-house determination of specific PAHs, particularly B $[a] \mathrm{P}$ by BELL (20a) at Lorillard, OAKLEY and STAHR (266a) at Philip Morris, and WALKER (467) and STAMEY et al. $(423,424)$ at RJRT.

These and many other in-house reports on PAHs demonstrate that the early PAH research was not limited to

\footnotetext{
${ }^{\mathrm{d}}$ At the Internet address, http://legacy.library.ucsf.edu/cgi, by inserting the topic 'aromatic polycyclic hydrocarbons', one may access over 20 BAT and Brown and Williamson (B\&W) memoranda by CHAKRABORTY, THORNTON, and others on PAHs in tobacco smoke.
} 
academic or governmental laboratories or to laboratories at private institutions such as the Sloan-Kettering Institute, American Health Foundation, or Roswell Park Memorial Institute. Many of the above Tobacco Industry reports on PAHs may now be accessed at the Internet addresses cited in the references.

Additional PAHs - both tumorigenic and non-tumorigenic were subsequently identified in CSC but the level of $\mathrm{B}[a] \mathrm{P}$ in CSC could account for very little $(90,343,344,519)$ or less than $2 \%$ of the observed skin-painting effect $(495,519)$, the contribution of all the known tumorigenic PAHs in CSC could account for not much more than $3 \%$ of the observed effect. These findings led to the proposal by WYNDER and WRIGHT (519) that CSC contained a PAH that either possessed the same specific tumorigenicity as $\mathrm{B}[a] \mathrm{P}$ but was present at about 50 times the $\mathrm{B}[a] \mathrm{P}$ level or present in MSS was an unknown PAH that was "supercarcinogenic" compared to $\mathrm{B}[a] \mathrm{P}$, i.e., its specific tumorigenicity to mouse skin was 40 to 50 times that of $\mathrm{B}[a] \mathrm{P}$. After an 18-month search, WRIGHT, a colleague of WYNDER from the early to the late 1950s, concluded that neither type PAH was present in CSC. Subsequently, the absence of a "supercarcinogen" in CSC was confirmed by the identification of hundreds of PAHs in the PAH fraction of CSC by SNOOK et al. (419-422). Detailed examination of their lists does not reveal the presence of a PAH structurally different from any of those previously classified with regard to their specific tumorigenicity on mouse-skin painting.

No other CSC fraction possessed specific tumorigenicity to mouse-skin comparable to the PAH fraction. In the mid1950 s, the tumorigenicity of the $N$-nitrosamines in CSC was not an issue for several reasons: 1) The tumorigenicity of an $N$-nitrosamine was first defined in 1956 (233a), 2) The presence of $N$-nitrosamines in MSS was not suggested until the early 1960s (35a, 90a), and 3) Of the more than $300 \mathrm{~N}$-nitrosamines tested for tumorigenicity, only one type not found in tobacco smoke - the $N$-nitrosoalkylureas - was found to be tumorigenic to mouse skin [e.g., see Appendixes A-D in (282b)].

Consideration of all the tumorigenic PAHs and their levels in CSC could account for no more than $3 \%$ of the observed biological activity in mouse skin-painting studies. In 1961, WYNDER and HOFFMANN (495) stated:

The polynuclear aromatic hydrocarbons are mainly formed during the combustion of tobacco. The tobacco of our standard cigarettes contains only very minute quantities of benzo(a)pyrene [sic] $(0.02 \mathrm{ppm})$. A bioassay indicates that these polycyclic hydrocarbons of the condensate by themselves, however, can account for not more than 3 per cent of the total biological activity.

And in 1967, they reiterated their 1961 comment (513):

Without belaboring the point as to whether $\mathrm{B} a \mathrm{P}$ as such contributes to the carcinogenicity of tobacco smoke condensate, we can certainly agree that the concentration of $\mathrm{B} a \mathrm{P}$ may be regarded as an 'indicator' of carcinogenic PAH in tobacco smoke condensate ... While $\mathrm{B} a \mathrm{P}$ and other carcinogenic PAH can by themselves account for only a small portion of the total tumorigenic activity of cigarette smoke condensate, probably less than $2 \%$, they are, nevertheless, of obligatory importance as tumor initiators.

HOFFMANN and WYNDER (166) reported that the major carcinogenicity of CSC resided in the CSC fraction containing the bulk of the PAHs. However, the levels in CSC of the non-alkylated carcinogenic PAHs could explain no more than $1-3 \%$ of the observed activity. They also reported that the artificial doubling and tripling of the levels of the 17 known tumorigenic PAHs in CSC significantly increased the tumorigenicity of the CSC. However, their biological findings were contradicted by those of ROE (343, 344) and LAZAR et al. (214) who reported that increasing the level of $\mathrm{B}[a] \mathrm{P}$ in $\mathrm{CSC}$ by a factor of 10 or 30 , respectively, produced no increase in the specific carcinogenicity of the CSC. RoE $(343,344)$ also noted that the CSC level of $\mathrm{B}[a] \mathrm{P}$, despite its known tumorigenic potency, accounted for very little of the observed specific tumorigenicity of CSC to mouse skin. The opposite of these observations were the findings that potently tumorigenic PAHs such as $\mathrm{DB}[a, h] \mathrm{A}$ on subcutaneous injection [DOBROVOLSKAIAZAVADSKAIA (88a)] and $\mathrm{B}[a] \mathrm{P}$ on mouse skin painting [POEL et al. (282a)] exhibited a threshold value. WYNDER et al. (486) reported that mice skin painted with the equivalent of the $\mathrm{B}[a] \mathrm{P}$ content of the CSC from over 500 current cigarettes developed no carcinomas. Rabbits were found to be even more resistant to higher dose levels of $\mathrm{B}[a] \mathrm{P}$.

Paralleling the research on the presence or absence of PAHs in cigarette MSS, their precursors in tobacco, their mechanism of formation, their contribution to laboratory animal tumorigenesis, and their possible involvement in the smoking-health issue was extensive research on ways to generate a "less hazardous" cigarette by removal of PAHs from or reduction of their per cigarette yields in MSS. To successfully resolve these questions, much pioneering research and development were initiated in late 1954 (323). When the question of the presence of PAHs in MSS was resolved, with many PAHs identified, and their per cigarette MSS yields determined, much effort was expended to develop technologies to reduce their MSS yield, particularly the yields of those PAHs reported to be tumorigenic to CSC-painted mouse skin. In the early 1960s, a "less hazardous" cigarette was defined on the basis of three criteria [see p. iii in (116); p. 372 in (500); pp. 503, 531 in (508)]: 1) the per cigarette yield of a specific toxicant has been lowered, 2) the ratio of the specific toxicant to MSS 'tar' has been lowered, and 3) the specific tumorigenicity of the MSS 'tar' as measured in the mouse skin-painting bioassay has been lowered. With the advent of meaningful tests for mutagenicity and genotoxicity, criterion 3 has been modified to include them.

The Tobacco Industry and non-Industry scientists investigated many additional approaches in the attempt to design a "less hazardous" cigarette [see Table 5 in (323), Table 14 in (336)]. Two examples of technologies that appeared to be promising but presented other toxicant problems were 1) the organic solvent-extraction of tobacco and 2) the use of oxidative additives.

The extraction concept was patterned after the findings of ROFFO (350) with one addition, the hexane extract of the tobacco was partitioned between hexane and aqueous ethanol to separate the flavorful compounds from those considered to be the PAH precursors, i.e., the phytosterols, the aliphatic hydrocarbons, the long-chained terpenoids $(9$, 10). When the extracted tobacco was smoked in cigarette form, its CSC showed much lower PAH levels than the control tobacco CSC $(309,314,521)$ and reduced tumorigenicity (521). The flavorful components, when returned to the extracted tobacco and smoked in cigarette form, 
contributed little to the total PAHs or $\mathrm{B}[a] \mathrm{P}$ in the MSS [see Figure 1, Table 3, and accompanying text in (323)]. The solvent extraction removed from the tobacco not only many of the PAH precursors but also much of several potent anticarcinogens to such tumorigens as $\mathrm{B}[a] \mathrm{P}$ and $\mathrm{DB}[a, h] \mathrm{A}$, e.g., long-chained aliphatic hydrocarbons, $d$ limonene, $\alpha$-tocopherol, $\alpha$ - and $\beta$-1,5,9-trimethyl-12-(1methylethyl)-4,8,13-cyclodecatriene-1,3-diol [(see Table 11 in (336)]. Thus, because of their removal from the tobacco, the anticarcinogens obviously could not be transferred to MSS during smoking. Before some of the problems were discovered, the investigation of the benefits supposedly derived from the organic solvent-extraction of tobacco led to several patents on the technology $(10,251,253)$. The earliest major non-Tobacco Industry proponents of the contribution of the extraction technology to a "less hazardous" cigarette eventually dismissed it with the comment that the technology was "impractical both technically and economically" (494) and "of academic interest only" (489). Most of the findings on tobacco components that were, and tobacco components that were not, significant precursors of MSS PAHs in this early study were confirmed some years later by SEVERSON et al. (398). The problems arising from the organic solvent extraction included the increased levels of nitrate and the biopolymers cellulose, starch, and pectin in the solvent-extracted tobacco. These consequences increased the yields of nitric oxide, $N$-nitrosamines, and phenols (331a) in the MSS.

While nitrate addition reduced the per cigarette yields of FTC 'tar', MSS PAHs, phenols, and CSC tumorigenicity to mouse skin (164), it was subsequently shown, as predicted (165), to significantly increase the yields of MSS $N$ nitrosamines and nitrogen oxides (39). Thus, the recommendation to add nitrate to tobacco to reduce MSS PAHs was eventually replaced by the recommendation to use lownitrate tobacco in the cigarette blend and/or remove nitrate from the tobacco (165). This reversal of recommendations was paralleled by another concerning the level of longchained hydrocarbons such as $n$-hentriacontane in tobacco: Originally, it was proposed to reduce MSS PAHs by selection of tobaccos with low levels of such components or remove the PAH precursors by organic solvent extraction. This was replaced by a proposal to select tobaccos with high levels of such components (39).

By the early 1960s, several cigarette design technologies developed by the Tobacco Industry and used in commercial products were categorized as significant in their contribution to the "less hazardous" cigarette (493). Ultimately, the initial four design technologies (tobacco blend, effective and efficient filtration, reconstituted tobacco sheet (RTS), air dilution via cigarette paper porosity) were increased to eight [tobacco blend, filter tip, filter tip additives, RTS, paper additives, expanded tobacco, air dilution (paper porosity), air dilution (filter tip perforation)]. Their significance was recognized in "less hazardous" cigarette design by the NATIONAL CANCER INSTITUTE (NCI) $(245)^{\mathrm{e}}$ and the

\footnotetext{
${ }^{\mathrm{e}}$ All eight cigarette design technologies eventually classified as significant by NCI, several US Surgeon Generals, and other investigators on the basis of the 10-year NCI Smoking and Health Program on the "less hazardous" cigarette had been incorporated into one or more US commercial cigarette products prior to the first meeting of the Tobacco Working Group formed in 1968 for the NCI program. In other words,
}

US Surgeon General [see Table 6 in (323), Table 15 in (336)] (445-453). It should be noted that the first two technologies considered significant were used before 1954 . Tobacco or tobacco blend selection had been used since 1913, even before the first tumors were induced in a laboratory animal by skin painting with a solution of coal $\operatorname{tar}(522)$. RTS was introduced into cigarette blends in 1953 when little was known about the chemical composition or biological properties of tobacco smoke (194) or the effect of RTS inclusion in the blend on them. When knowledge of tumor induction with CSC and the presence of PAHs including $\mathrm{B}[a] \mathrm{P}$ became available, it was shown that use of these two technologies resulted in a cigarette whose MSS was in compliance with that in the definition of a "less hazardous" cigarette (336).

Of course, the initial thrust of this across-the-board reduction was aimed at reducing the MSS 'tar' delivery because of extrapolation by WYNDER et al. (516) of their 1957 mouse-skin bioassay findings:

Although it is difficult to estimate a comparable exposure level for man, the human data in line with the animal data indicate that a reduction in total tar exposure will be followed by a decrease in tumor formation. For this reason, measures directed toward this reduction are of utmost importance ... The minimum dose of tar capable of producing papillomas in mice is about one third, of producing cancer one half, that of the optimum dose ... The practical implications of these data and their relationship to the human cancer problem have been emphasized.

In his 1957 testimony during the filter-tipped cigarette hearings, WYNDER reiterated this opinion that reducing 'tar' exposure dose by $40 \%$ to $50 \%$ would substantially reduce lung cancer induction in smokers (483):

Examination of the sales-weighted average 'tar' delivery for US commercial cigarettes reveals that the $40 \%$ to $50 \%$ reduction in MSS 'tar' delivery considered vital by WYNDER in 1957 was achieved in the late 1960s, i.e., a reduction from $38-39 \mathrm{mg} / \mathrm{cig}$ to $19-20 \mathrm{mg} / \mathrm{cig}$. Further examination reveals that by the early 1980 s, the salesweighted average 'tar' was reduced to about $12 \mathrm{mg} / \mathrm{cig}$, i.e., an additional $40 \%$ reduction had been achieved [see Figure 3 in (323)]. Corresponding reductions in the per cigarette yields of total PAHs in general, $\mathrm{B}[a] \mathrm{P}$ in particular (470), and nicotine were also observed. These reductions were also accompanied by a reduction in the specific tumorigenicity (mouse-skin painting) of the MSS CSC (448). By year-end 1963, 91 of the 97 PAHs identified in MSS were reported in the published literature. Six PAHs, identified in MSS by RODGMAN and COOK (329), had not been reported publicly at that time. However, by 1970 , identification in MSS of all but one (cholanthrene) of the 97 had been reported. Despite the availability of such information, only 18 MSS PAHs were discussed by the Advisory Committee in its 1964 Report to the US Surgeon General, 13 as mainstream CSC components and five as carbon black components (445). The detailed discussion of so few MSS PAHs and citation of so few publications was done despite the fact the Committee had been provided with a detailed Philip Morris monograph on tobacco and smoke composition, a monograph that listed $61 \mathrm{PAHs}$

from 1968 to 1978 , no new design technology was generated in the NCI Smoking and Health Program on the "less hazardous" cigarette. 


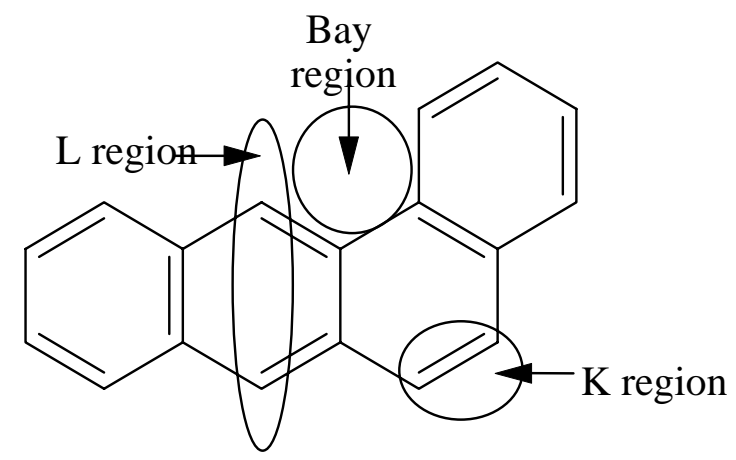

Figure 1. The $L$ region, $K$ region, and bay region of benz[a]anthracene

identified in tobacco smoke plus many pertinent published references to them $(278,323)$. The Advisory Committee did note, however, that 27 other non-tumorigenic PAHs - none specifically named - had been identified in tobacco smoke. The 27 unnamed PAHs had to include several of those PAHs, e.g., naphthalene, anthracene, phenanthrene, fluoranthene, pyrene, which had been reported to significantly inhibit the action of potently tumorigenic PAHs such as $\mathrm{B}[a] \mathrm{P}$ and $\mathrm{DB}[a, h] \mathrm{A}$ in laboratory animal studies. Of the 97 PAHs known to him, RoDGMAN (323) discussed the 43 PAHs identified at RJRT plus 34 other PAHs reported in the literature in numerous reports between 1954 and 1964 and in a summary 1964 report on 10-year research on cigarette MSS (317). Interestingly, Chapter 6, in the Advisory Committee's report on cigarette smoke chemistry and the tumorigenic PAHs, was primarily authored by FIESER, one of the two eminent American PAH authorities at that time.

\section{THE CATALOGUE OF POLYCYCLIC AROMATIC HYDROCARBONS: A REASON FOR IT}

For over half a century, numerous theories have been advanced in attempts to explain the relationship between the tumorigenicity of polycyclic aromatic hydrocarbons (PAHs) in treated laboratory animals and a variety of their structural properties, including such properties as their K-, L-, and bayregions, electron distribution, bond orders, bond strengths, resonance, octanol-water partitioning, and the like, Figure 1. Such studies were triggered by the discovery that certain PAHs when administered to laboratory animals via skin painting or subcutaneous injection induced carcinomas or sarcomas, respectively. DB $[a, h] \mathrm{A}$, synthesized independently by CLAR (58) and FIESER and DIETZ (105a) in 1929, was shown to be a potent tumorigen to laboratory animals by KENNAWAY and HIEGER (186). Shortly thereafter, COOK et al. (64) isolated several PAHs from coal tar, characterized one of them as the previously unknown ben$\mathrm{zo}[a]$ pyrene $(\mathrm{B}[a] \mathrm{P})$, and demonstrated that it too was a potent tumorigen to laboratory animals (18). Over the next two decades, the first demonstrations of the carcinogenicity of two pure compounds, $\mathrm{DB}[a, h] \mathrm{A}$ and $\mathrm{B}[a] \mathrm{P}$, led to the synthesis and subsequent testing for tumorigenicity in laboratory animals of literally hundreds of PAHs and their alkyl derivatives plus other derivatives.
During this time, the variation in biological responses observed with laboratory animals to individual PAHs eventually led to numerous unacceptable extrapolations of the results to PAH-exposed humans. To put the laboratory animal-to-human extrapolation in perspective, SHEAR and LEITER (404) in 1941 issued a list of pertinent factors to be considered in such an extrapolation. Despite a diminution in PAH synthesis and tumorigenicity research during World War II, the wealth of experimental data available in the late 1940s - early 1950s on the high-to-slight tumorigenic potency of some PAHs and the non-tumorigenicity of other PAHs induced investigators to seek reasons for the observed differences in tumorigenicity and to attempt to develop explanations for them. Among those involved in the generation of the major early theories on the relationship between PAH structural properties and PAH tumorigenicity or lack of it were COULSON (71); PULLMAN and PULlMAN (283); DAUDEL and DAUDEL (77); FIESER $e t$ al. (102) and FIESER (103); and LACASSAGNE et al. (204). Much meaningful input to these theories was provided by other investigators such as PAULING (275a) in the USA, BOYLAND, WEIGERT, and MOTTRAM (35b) in the UK, and BUU-HoÏ in France [see more than 30 BUU-HoÏ references listed in (204)]. More recent studies include those by HERNDON et al. (150, 231), RUBIN (356), TROSKO (440), L. ZHANG et al. (529), and Y. ZHANG, a graduate student under HERNDON (530).

Because it was issued at the beginning of the extensive research on the composition of tobacco smoke with particular emphasis on the nature and levels of the PAHs in it, it is interesting to examine the lengthy 1955 review by PULLMAN and PULLMAN (283) on the relationship between electronic structure and the tumorigenicity of a number of benzenoid hydrocarbons. Their publication was a detailed update of the 1953 review by COULSON (71) and included much data generated in the interim. The PULLMANs used calculations based on three theoretical indexes of the $\mathrm{K}$ and $\mathrm{L}$ regions of the aromatic hydrocarbons. The indexes included Carbon Localization Energy (CLE), Bond Localization Energy (BLE), and Para Localization Energy (PLE) [see Table 1 in (283)]. The PULLMANs, by use of their CLE, BLE, and PLE calculations pertinent to the $K$ and $L$ regions in the PAHs, also attempted to relate the structures of various PAHs and their alkylated derivatives not only to their tumorigenicity but also to their rate of reaction in certain well-known reactions, e.g., Diels-Alder reaction with maleic anhydride, reaction with osmium tetroxide, reaction with lead tetraacetate, photo-oxidation. Table 2 lists the hydrocarbons discussed by the PULLMANs in 1955 with an indication of those, 34 in all, which were identified in tobacco smoke before and after 1955.

The PULlmans did introduce into their discussion various PAH metabolites, their diols and phenols, but not the epoxides which were unknown at that time. Even though it had been known since 1951 (426), no explanation was offered for the inhibition of the activity of a potently tumorigenic PAH by co-administration of a weakly tumorigenic or non-tumorigenic PAH. Lastly, of course, neither the PULLMANs nor COULSON discussed the fact that a bioassay finding with a highly susceptible strain or species of laboratory animal administered an individual $\mathrm{PAH}$ in an excessive dose has little relationship to the 
Table 2. Benzenoid hydrocarbons discussed by PULLMAN and PULLMAN (283)

\begin{tabular}{|c|c|c|c|c|}
\hline Aromatic hydrocarbon discussed & CAS RN & $\begin{array}{l}\text { No. in PAH list in } \\
\text { Table } 6 \\
\end{array}$ & $\begin{array}{c}\text { No. in Pullman and } \\
\text { Pullman }\end{array}$ & $\begin{array}{c}\begin{array}{c}\text { Considered tumorigenic } \\
\text { in } 1955\end{array} \\
\end{array}$ \\
\hline \multicolumn{5}{|l|}{ Monocyclic } \\
\hline Benzene $^{\mathrm{b}}$ & $71-43-2$ & - & I & no \\
\hline \multicolumn{5}{|l|}{ Bicyclic } \\
\hline Naphthalene & $91-20-3$ & 1 & II & no \\
\hline \multicolumn{5}{|l|}{ Tricyclic } \\
\hline Anthracene & $120-12-7$ & 157 & III & no \\
\hline Phenanthrene & $85-01-8$ & 173 & IV & no \\
\hline \multicolumn{5}{|l|}{ Tetracyclic } \\
\hline Naphthacene & $92-24-0$ & 264 & VII & no \\
\hline Benz[a]anthracene & $56-55-3$ & 266 & VI & $?$ \\
\hline Benz[a]anthracene, 2,10-dimethyl- & - & - & XLIII & $?$ \\
\hline Benz[a]anthracene, 7,12-dimethyl- & $57-97-6$ & 270 & XLII & yes \\
\hline Benz[a]anthracene, 7-methyl- & $2541-69-7$ & - & XLIV & yes \\
\hline Benzo[c]phenanthrene & $195-19-7$ & 290 & $\mathrm{~V}$ & yes \\
\hline Benzo[c]phenanthrene, 1,2-dimethyl- & - & - & XLVIII & no \\
\hline Chrysene & $218-01-9$ & 292 & VIII & $?$ \\
\hline Chrysene, 2,3-dimethyl- & - & $294^{c}$ & XLIX & no \\
\hline Triphenylene & $217-59-4$ & 307 & $x$ & no \\
\hline Pyrene & $129-00-0$ & 311 & IX & no \\
\hline \multicolumn{5}{|l|}{ Pentacyclic } \\
\hline Benzo[b]chrysene & $214-17-5$ & 414 & XXIII & no \\
\hline Benzo[c]chrysene & $194-69-4$ & - & XIII & yes \\
\hline Benzo[g]chrysene & $196-78-1$ & - & XIV & yes \\
\hline Pentacene & $135-48-8$ & 415 & XVIII & no \\
\hline Benzo[a]naphthacene & $226-88-0$ & 416 & $\mathrm{XVII}$ & no \\
\hline Dibenz $[a, h]$ anthracene & $53-70-3$ & 418 & XII & yes \\
\hline Dibenz $[a, j]$ anthracene & $224-41-9$ & 419 & $X V$ & yes \\
\hline Pentaphene & $222-93-5$ & 420 & $\mathrm{XIX}$ & no \\
\hline Perylene & $198-55-0$ & 421 & $X X V$ & no \\
\hline Picene & $213-46-7$ & 427 & $X X I$ & no \\
\hline Benzo[b]triphenylene & $215-58-7$ & 428 & $x x$ & no \\
\hline Benzo[a]pyrene & $50-32-8$ & 430 & $\mathrm{XI}$ & yes \\
\hline Benzo[a]pyrene, 2-methyl- & - & $437-438^{d}$ & XLVII & yes \\
\hline Benzo[a]pyrene, 3-methyl- & - & - & XLVII & yes \\
\hline Benzo[a]pyrene, 5-methyl- & - & - & XLVII & yes \\
\hline Benzo[a]pyrene, 6-methyl- & $2381-39-7$ & - & XLVII & yes \\
\hline Benzo[a]pyrene, 7-methyl- & $63041-77-0$ & - & XLVII & yes \\
\hline Benzo[a]pyrene, 8-methyl- & $63041-76-9$ & - & XLVII & no \\
\hline Benzo[a]pyrene, 9-methyl- & - & - & XLVII & no \\
\hline Benzo[e]pyrene & $192-97-2$ & 440 & $\mathrm{XVI}$ & no \\
\hline Dibenzo $[b, g]$ phenanthrene & $195-06-2$ & - & XXIV & no \\
\hline Dibenzo[c,g]phenanthrene & $188-52-3$ & - & XXII & no \\
\hline Benz[]]aceanthrylene, 1,2-dihydro-3-methyl- e & $56-49-5$ & 449 & XLV & yes \\
\hline \multicolumn{5}{|l|}{ Hexacyclic } \\
\hline Anthra[1,2-a]anthracene & $195-00-6$ & - & $X X X V$ & no \\
\hline Benzo[c]pentaphene & $222-54-8$ & - & XXXVII & no \\
\hline Benzo[rst]pentaphene & $189-55-9$ & 482 & LIV & $(\text { yes })^{f}$ \\
\hline Benzo[pqr]picene & $189-96-8$ & - & LVII & no \\
\hline Dibenzo $[b$, def $]$ chrysene & $189-64-0$ & 493 & XXVII & yes \\
\hline Dibenzo $[b, k]$ chrysene & $217-54-9$ & - & XXXVI & no \\
\hline Dibenzo[c,mno]chrysene & $196-28-1$ & - & LVI & no \\
\hline Dibenzo[def,mno]chrysene & $191-26-4$ & 494 & XXXI & no \\
\hline Dibenzo[def,p]chrysene & $191-30-0$ & 501 & XXVI & yes \\
\hline Dibenzo[a,j]naphthacene & $227-04-3$ & 504 & XXXIII & no \\
\hline Dibenzo[a,]naphthacene & $226-86-8$ & - & XXXIV & no \\
\hline Dibenzo[fg,op]naphthacene & $192-51-8$ & 506 & XXIX & no \\
\hline Naphtho[1,2,3,4-def]chrysene & $192-65-4$ & 508 & XXVIII & $?$ \\
\hline Naphtho[2,1,8-qra]naphthacene & $196-42-9$ & 510 & $X X X$ & no \\
\hline Naphtho[1,2-b]triphenylene & $215-26-9$ & 511 & XXXII & no \\
\hline
\end{tabular}




\begin{tabular}{|c|c|c|c|c|}
\hline Aromatic hydrocarbon discussed & CAS RN & $\begin{array}{l}\text { No. in PAH list in } \\
\text { Table } 6\end{array}$ & $\begin{array}{l}\text { No. in Pullman and } \\
\text { Pullman }\end{array}$ & $\begin{array}{c}\text { Considered tumorigenic }^{a} \\
\text { in } 1955\end{array}$ \\
\hline \multicolumn{5}{|l|}{ Heptacyclic } \\
\hline Benzo[a]naphtho[8,1,2-Imn]naphthacene & $190-01-2$ & - & LV & no \\
\hline Dibenzo[fg,qr]pentacene & $197-74-0$ & - & $X L$ & no \\
\hline \multicolumn{5}{|l|}{ Octacyclic } \\
\hline Dinaphtho[1,2-b:1,2-k]chrysene & $214-13-1$ & - & XXXIX & no \\
\hline Naphthaceno[2,1,12,11-opqra]naphthacene & $188-42-1$ & - & LVIII & $?^{g}$ \\
\hline Phenanthro[1,10,9,8-opqra]perylene & $190-39-6$ & - & $\mathrm{XLI}$ & no \\
\hline \multicolumn{5}{|l|}{ Nonacyclic } \\
\hline Dinaphtho[1,2-b:1,2-n]perylene & - & - & XXXVIII & no \\
\hline \multicolumn{5}{|l|}{ Decacyclic } \\
\hline Pentacenopentacene & - & - & LIX & $?^{9}$ \\
\hline
\end{tabular}

a Tumorigenic in mouse skin-painting study.

${ }^{\mathrm{b}}$ Benzene was reported as a component of the vapor phase of tobacco smoke in 1955 by RESNIK and HOLMES (298a) and LAURENE (212a).

${ }^{\mathrm{C}} \mathrm{A}$ dimethylchrysene was reported in tobacco smoke, but the positions of the methyl groups were not defined.

${ }^{d}$ At least two methylB[a]Ps were reported in tobacco smoke, but the position of the methyl group in each case was not defined.

${ }^{\mathrm{e}}$ This PAH is not totally benzenoid; its structure includes a cyclopentanoid ring:

${ }^{f}$ In 1955, the tumorigenicity of dibenzo[rst]chrysene had not been determined; later it was reported to be tumorigenic.

${ }^{9}$ Although no calculation was made on this PAH, PULLMAN and PULLMAN (283) predicted it would be tumorigenic.

Table 3. Polycyclic hydrocarbons reported in tobacco smoke by year-end 1955

\begin{tabular}{|c|c|c|c|c|}
\hline \multirow[b]{2}{*}{ Hydrocarbon } & \multicolumn{4}{|c|}{ References issued in the year } \\
\hline & 1947 & 1953 & 1954 & 1955 \\
\hline $\begin{array}{l}\text { Acenaphthylene }{ }^{a} \\
\text { Azulene }^{a, b}\end{array}$ & 173 & 67 & 68,70 & $\begin{array}{c}69,221 \\
221\end{array}$ \\
\hline Anthracene & & 67 & 61,68 & $\begin{array}{l}69,219 \\
221,395\end{array}$ \\
\hline Anthracene, 2-methyl- & & & & 69 \\
\hline Benz[a]anthracene & & & & 219,227 \\
\hline Benzo[ghi]perylene & & & 68 & $\begin{array}{c}69,221 \\
227\end{array}$ \\
\hline Benzo[a]pyrene & & & $\begin{array}{l}61,68 \\
70,217\end{array}$ & $\begin{array}{c}6,48,100 \\
184,221 \\
395,518\end{array}$ \\
\hline Benzo[e]pyrene & & & & 219 \\
\hline Dibenzo[def,mno]chrysene & & & 68 & 69,221 \\
\hline Fluoranthene $^{\mathrm{a}}$ & & & 68 & 69,221 \\
\hline Naphthalene & & & & 395 \\
\hline Naphthalene, 2-methyl- & & & & 69 \\
\hline Phenanthrene & & & 68 & 69,221 \\
\hline Pyrene & & 67 & 61,68 & $\begin{array}{l}69,219 \\
221,227\end{array}$ \\
\hline
\end{tabular}

\footnotetext{
${ }^{\text {a } M o l e c u l e ~ h a s ~ a ~ c y c l o p e n t a n o i d ~ r i n g, ~ t h u s ~ i t ~ w a s ~ n o t ~ c o n s i d e r e d ~}$ by PULLMAN and PULLMAN (283).

${ }^{\mathrm{b}}$ Molecule does not possess a benzenoid structure.
}

situation where a human is exposed by a different administration route to a mixture of PAHs with various degrees of tumorigenicity plus other known anti-tumorigenic compounds.

By year-end 1955, very few of the PAHs considered by the PULLMANs had been reported as tobacco smoke components. More had been identified in other sources such as air pollution. In the following discussion, the comments in the early 1950s about the inadequacy of the evidence indicating the presence of $\mathrm{B}[a] \mathrm{P}$ in tobacco smoke (104) are disregarded. Table 3 lists the PAHs reported in tobacco smoke at that time. Of the 14 PAHs reported, only eight were included by the PULLMANs in their assessment: naphthalene, anthracene, phenanthrene, $\mathrm{B}[a] \mathrm{A}, \mathrm{B}[a] \mathrm{P}, \mathrm{B}[e] \mathrm{P}$, dibenzo[def,mno $]$ chrysene, naphthalene, phenanthrene, and pyrene. Of course, only one of the eight, $\mathrm{B}[a] \mathrm{P}$, was considered at that time a significant and potent tumorigen to mouse skin. At that time, the tumorigenicity of $\mathrm{B}[a] \mathrm{A}$ was questioned, and still was questioned in the mid-1980s (88).

As noted previously, Pullman and Pullman not only updated the electronic structure-tumorigenicity information generated after the COULSON 1953 review but also attempted to extend the theory to alkyl-PAHs. Examination of their review reveals that they discussed, in addition to 1,2-dihydro-3-methylbenz[j] aceanthrylene, a total of twelve alkyl-PAHs (see Table 3). It is obvious from their discussion that the prediction of tumorigenicity for most of these 12 PAHs was not calculated but derived from published biological data. However, examination of the biological data in HARTWELL (144) and SHUBIK and HARTWELL (408) indicates that at least 64 totally benzenoid alkyl-PAHs had been tested for tumorigenicity by 1955 . Several 1,2dihydromethylbenz[j]aceanthrylenes had been tested for tumorigenicity by 1955 , but they were not included in our count of 64 . This raises the question: Why was the prediction not calculated for more of the 64 alkyl-PAHs, the tumorigenicity of which was known at that time (144, 408)? PULLMAN and PULLMAN noted:

It must be acknowledged that the extension of the theory to substituted derivatives of polycyclic hydrocarbons is at present far from having achieved a completely consistent and satisfactory form.

Many of the more recent theories on the relationship between PAH structural properties and tumorigenicity suffer somewhat from this and other deficiencies [cf. HERNDON et al. (150, 231), RUBIN (356), Trosko (440), L. ZHANG et al. (529), and Y. ZHANG (530)]. Much studied in recent years has been the application of the quantitative structure-activity relationship (QSAR) method to PAHs.

While many theories have involved the relationships between observed laboratory-derived biological data on individually administered PAHs and their structural 
elements, do they speak to the exposure situation experienced by humans? Whether the exposure is by inhalation of air pollutants or tobacco smoke, by ingestion of foodstuffs or beverages, by dermal contact, or by a combination of the exposures, very few of any human exposures involve exposure to a single PAH similar to the exposure of laboratory animals treated with a single PAH by skin painting or subcutaneous injection. One such example of human exposure to a single $\mathrm{PAH}$ was the past use of naphthalene as the major ingredient in mothballs.

Numerous PAHs have been either completely of partially characterized in many air pollutants, foodstuffs, beverages, and contact tars and dusts. Of all the products to which humans are exposed, none has been characterized to the extent of tobacco smoke. Nearly 4800 components have been identified in it, nearly twice as many as in the next consumer product, coffee, subjected to detailed compositional analysis. Of the identified tobacco smoke components, about $11 \%$ were either completely or partially identified as PAHs. It should also be noted that in the detailed examination of tobacco smoke, the 4800 identified components account for over $98 \%$ of the weight of cigarette MSS. It has been estimated, based on detailed gas chromatograms, that the number of actual components in cigarette MSS may be 12 to 20 times the number of identified ones (465).

Of the more than 500 PAHs either completely or partially identified in cigarette MSS, relatively few PAHs, originally 13 in all, were repeatedly defined as significant tumorigens (152, 167, 174). Eventually, the International Agency for Research on Cancer (IARC) redefined the tumorigenicity of chrysene. Thus, it was deleted from all subsequent lists $(153,154,156,157,268)$ except one (106). MSS is not the only source of most of the 12 PAHs still considered as significant tumorigens in cigarette MSS. Except for 5methylchrysene, most have also been identified as significant PAH components of gasoline and diesel engine exhaust gases $(132,496)$ and many common foodstuffs and beverages $(122,232)$.

When one is dealing with a complex mixture, which in turn contains an assortment of PAHs ranging from bicyclic to decacyclic, one cannot extrapolate the biological effect observed by administration of an individual PAH to the biological effect of that PAH in such a complex mixture. It has long been known from laboratory studies that certain non-tumorigenic or slightly tumorigenic PAHs when administered by skin painting or subcutaneous injection in an equimolar dose level with a highly tumorigenic $\mathrm{PAH}$ partially or totally inhibit its tumorigenicity. Few studies have been done to determine the effect of a non- or lowtumorigenic PAH on the tumorigenicity of a highly tumorigenic PAH when its level greatly exceeds that of the potent tumorigen. Also, there are differences in the classification of the potency of the tumorigenicity of some PAHs. For example, $\mathrm{B}[a] \mathrm{A}$ is classified by some (174) as a potent or significant tumorigen but by others as only slightly tumorigenic $(88)^{\mathrm{f}}$.

${ }^{\mathrm{f}}$ One of us (A.R.) was involved in the late 1940s in a comparison of the tumorigenicities of several PAHs $(\mathrm{B}[a] \mathrm{P} ; \mathrm{DB}[a, h] \mathrm{A} ; \mathrm{B}[a] \mathrm{A})$ administered by skin painting or by subcutaneous injection. Equimolar doses of each PAH were administered to groups of mice (50 per group) so that the $\%$ Tumor Bearing Animals (TBA) with $\mathrm{B}[a] \mathrm{P}$ and $\mathrm{DB}[a, h] \mathrm{A}$ were approximately $80 \%$. The equimolar dose of $\mathrm{B}[a] \mathrm{A}$, a commercial sample, m.p. $166-167{ }^{\circ} \mathrm{C}$, gave only $2 \%$ TBA, i.e., one mouse with tumor.
The list of either totally or partially identified PAHs in CSC gradually increased but in the mid-1970s the massive definitive PAH study by United States Department of Agriculture (USDA) personnel in Athens, GA increased the number of known PAHs in CSC to well over 500 (416, 417-422). Although not isolated individually, their identifications, whether total or partial, have generally been accepted across the board.

Numerous authors, including HOFFMANN and HECHT (152), listed the PAH dibenzo[ $[a, l]$ pyrene as a significant tumorigen in tobacco smoke. However, HECHT eventually stated (145) that "the presence in cigarette smoke of dibenzo[ $[a, l]$ pyrene, a highly carcinogenic PAH, had not been confirmed". One should weigh the comment by HECHT against the current status of defined MSS composition. Since the appreciable decline in detailed tobacco smoke composition studies after the late 1970s, no individual investigator or no research group has reported the confirmation of the identities of many of the PAHs (419-422), aza-arenes (418, cf. 362), nitrogen-containing components (147), or ether- (264) and water-soluble components (391) reported in cigarette MSS in the 1970s. While many components have been confirmed by other investigators at the same institution as the authors, examination of the post-1980 literature indicates that the identities of nearly half the new components described in the above-mentioned studies have not been confirmed by investigators at other institutions. Because of such a situation, would HECHT also discount their presence in cigarette MSS in the same way as he discounted the presence of dibenzo $[a, l]$ pyrene?

While most of the past theories have attempted to define the relationship between structural properties of the PAHs and their specific tumorigenicity as measured individually in skin-painting studies, little has been done to explain the behavior of a PAH when it is present in a complex mixture which includes a host of PAHs some of which are known anti-tumorigens as well as numerous known non-PAH antitumorigens (101).

It has been known for over 60 years that co-administration of a potently tumorigenic $\mathrm{PAH}$ with an equimolar quantity of a non-tumorigenic PAH often results in substantial reduction in \% tumor bearing animals (\% TBA).

In 1953, COULSON noted [see p. 51 in (71)]:

The action of inhibitors may be thought of as a competition between the carcinogenic and noncarcinogenic compounds for available sites on the enzyme. If sufficient noncarcinogenic molecules are able to occupy suitable sites, then the irreversible mutation cannot occur. We can see that inhibitors, in order to compete with the carcinogenic compounds, should themselves possess a K-region.

Some of the PAHs that substantially reduce or totally inhibit the tumorigenicity of several of the most potent tumorigens known are listed in Table 4. Obviously, neither naphthalene nor anthracene has a K-region, a requirement proposed by COULSON for the inhibitory property.

Purification of the $\mathrm{B}[a] \mathrm{A}$ by complex formation and column chromatography on alumina not only increased the melting point and diminished the m.p. range $\left(167.2-167.5{ }^{\circ} \mathrm{C}\right)$, but improved the $\mathrm{UV}$ absorption spectrum. An equimolar dose of the purified $\mathrm{B}[a] \mathrm{A}$ gave $0 \%$ TBA; quintupling the dose gave $0 \%$ TBA. The following question remained unanswered: Was the $2 \%$ TBA with the commercial sample due to the $\mathrm{B}[a] \mathrm{A}$ or to a contaminant? 
Table 4. Inhibition of tumorigenicity of potently tumorigenic PAHs by non-tumorigenic or weakly tumorigenic PAHs

\begin{tabular}{l|r|l|l}
\hline PAH $^{a}$ & CAS No. & Effective against & References \\
\hline Naphthalene & $91-20-3$ & $\mathrm{~B}[a] \mathrm{P}, \mathrm{DB}[a, h] \mathrm{A}$ & 72 \\
Anthracene & $120-12-7$ & $\mathrm{~B}[a] \mathrm{P}, \mathrm{DB}[a, h] \mathrm{A}$ & 72 \\
Phenanthrene & $85-01-8$ & $\mathrm{DMB}[a] \mathrm{A}$ & 82,410 \\
Fluoranthene & $206-44-0$ & $\mathrm{~B}[a] \mathrm{P}, \mathrm{DMB}[a] \mathrm{A}$ & $82,410,411$ \\
Pyrene & $129-00-0$ & $\mathrm{DB}[a, h] \mathrm{A}, \mathrm{DMB}[a] \mathrm{A}$ & $82,410,411$ \\
Benz[a]anthracene & $56-55-3$ & $\mathrm{~B}[a] \mathrm{P}, \mathrm{DB}[a, h] \mathrm{A}$ & 426,509 \\
Benzo[e]pyrene & $192-97-2$ & $\mathrm{~B}[a] \mathrm{P}, \mathrm{DB}[a, h] \mathrm{A}, \mathrm{DMB}[a] \mathrm{A}$ & $82,410,411$ \\
Benzo[b]triphenylene & $215-58-7$ & $\mathrm{MC}^{\mathrm{b}}, \mathrm{DB}[a, h] \mathrm{A}, \mathrm{DMB}[a] \mathrm{A}$ & $82,409,411$ \\
\hline
\end{tabular}

${ }^{\mathrm{a}}$ Each PAH listed is a component of cigarette MSS. $\quad{ }^{\mathrm{b}} \mathrm{MC}=3$-methylcholanthrene = 1,2-dihydro-3-methylbenz[]]aceanthrylene.

Table 5. Levels of PAH classes in cigarette mainstream smoke

\begin{tabular}{|c|c|c|c|c|}
\hline \multirow[b]{2}{*}{ PAH category } & \multirow{2}{*}{$\begin{array}{c}\text { Assumed } \\
\text { approximate mol. wt. }\end{array}$} & \multicolumn{3}{|c|}{ Mainstream smoke yield ${ }^{a}$} \\
\hline & & Yield, ng/cig ${ }^{\mathrm{b}}$ & Approximate nanomoles ${ }^{\mathrm{C}}$ & Nanomolar ratio, $\mathrm{PAH}: \mathrm{B}[a] \mathrm{P}$ \\
\hline Bicyclic & $128^{d}$ & $4140(77.1)$ & 32.3 & 293 \\
\hline Tricyclic & $178^{e}$ & $720(13.4)$ & 4.0 & 36 \\
\hline Tetracyclic & 228 & $420(7.9)$ & 1.8 & 16 \\
\hline Pentacyclic & 278 & $72(1.3)$ & 0.26 & 2.4 \\
\hline $\mathrm{B}[\mathrm{a}] \mathrm{P}$ & 252 & $27(0.49)^{f}$ & 0.11 & 1.0 \\
\hline Non-B[a]P pentacyclic & 278 & $45(0.81)^{f}$ & 0.16 & 1.5 \\
\hline Hexacyclic & 328 & $14(0.3)$ & 0.04 & 0.36 \\
\hline TOTALS & & $5366(100.0)$ & & \\
\hline
\end{tabular}

${ }^{a}$ Data reported by HOFFMANN and WYNDER (163) from a nonfiltered cigarette, total particulate matter $=36.8 \mathrm{mg} / \mathrm{cig}$, were averaged with data reported by RODGMAN and COOK (329) for a filtered commercial cigarette, total particulate matter $=37.5 \mathrm{mg} / \mathrm{cig}$.

${ }^{\mathrm{b}}$ Values in parentheses represent the fraction \% of the PAH category in the total PAH fraction.

${ }^{\mathrm{C}}$ Nanomoles calculated with the approximate molecular weights in Column 2.

${ }^{d}$ The molecular weight of naphthalene $=128$, that of indene $=116$.It is realized that the average molecular weight of the bicyclic PAH mixture will differ slightly from those of the parent $\mathrm{PAH}$ because of the presence of numerous homologs (methylnaphthalenes, dimethylnaphthalenes, etc.).

${ }^{\mathrm{e}}$ The presence of tricyclic $\mathrm{PAH}$ homologs results in molecular weight slightly different from 178 .

${ }^{f}$ The sum of the fraction \% of $\mathrm{B}[\mathrm{a}] \mathrm{P}$ and the fraction \% of non-B[a]P pentacyclic PAHs equals $1.3 \%$.

While many of the inhibition studies were conducted with the tumorigenic and inhibiting PAHs administered in equimolar quantities, it should be remembered that this is not the case in the PAH mixture in CSC. Table 5 is derived from CSC PAH data presented by HOFFMANN and WYNDER $(163,165)$ and RODGMAN (329). The per cigarette yield data in Table 5 were the averages of the data generated from two different commercial American cigarettes. One was unfiltered and yielded 36.8 $\mathrm{mg} / \mathrm{cig}$ of total particulate matter (TPM) (163); the other was a filtered cigarette that yielded $37.5 \mathrm{mg} / \mathrm{cig}$ of TPM (329). The disparity between the relative yields in each category was less than $5 \%$.

In the early structure-biological activity studies, PAHs with a pentacyclic ring were not included in the discussion of most theories but pentacyclic compounds in which the pentacycle contained nitrogen were, i.e., benzacridines ( 71 , 204). In the discussion of his theory, Coulson (71) did mention several cyclopentanoid compounds: Six benzacridines and two PAHs, 2,3-dihydro- $1 H$-benzo[ $a]$ cyclopent $[h]$ anthracene and 10,11-dihydro-9H-benzo[a]cyclopent $[i]$ anthracene.

In her 1996 thesis, ZHANG (530) noted the numerous sources of PAHs to which humans are exposed, e.g., air pollutants, foodstuffs and beverages, effluents from factories, vehicles, and heat and power sources. ZHANG particularly stressed tobacco smoke, its complexity, and some of the PAHs contained therein:
Tobacco smoke is a complex mixture which is estimated to contain at least 150 compounds in the gas phase and more than 2000 compounds have been identified in the particulate phase. Table $1^{\mathrm{a}}$ lists some PAHs that exist in the particulate phase of cigarette smoke.

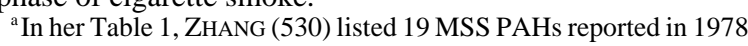
by HOFFMANN et al. (160a).

Unfortunately, the inconsistent use of PAH nomenclature sometimes makes it difficult to follow the phases of the study by ZHANG [cf. Table 7 and Appendix A in (530)].

Another study, recently initiated by MARTIN et al. (234a), involved an attempt to develop a meaningful relationship between PAH structure, chemical properties, and biological properties, specifically the effect of PAHs on specific tumorigenicity in skin painting. Reported for naphthaleneand pyrene-related PAHs were the following molecular parameters: The measured and calculated log of the octanolwater partition coefficient (MlogP, ClogP), molecular volume (MgVol), calculated molar refractivity (CMR), and the number of valence electrons (NVE). The second phase of the study involves similar data for anthracenes, phenanthrenes, and indenes (336a). All the PAHs in the first two phases of this study $(234 \mathrm{a}, 336 \mathrm{a})$ are reported components of cigarette MSS. The ultimate goal is to use these data to facilitate a quantitative structure-activity relationship (QSAR) on MSS PAHs. If such a meaningful relationship can be derived for the more than 500 MSS PAHs, then it probably can be applied to any PAH from any source. 
As a prelude to this attempt to develop a possibly reasonable explanation for the PAH structure-tumorigenicity relationship, the PAHs completely or partially identified in cigarette smoke have been catalogued. In Tables 6 and 7, the PAHs are categorized as bicyclic, tricyclic, tetracyclic, etc. with each group subdivided into all-benzenoid PAHs and cyclopentanoid-benzenoid PAHs. For each PAH, the nomenclature used in Tables 1, 4, and 5 is the most recent proposed by the International Union of Pure and Applied Chemistry (IUPAC).

The tobacco smoke PAH references cited in Table 6 are not necessarily all that are available, particularly for those PAHs such as $\mathrm{B}[a] \mathrm{P}$ and $\mathrm{DB}[a, h] \mathrm{A}$, which have been the subject of much research and discussion for over half a century. In most cases, included is a reference to the publication or presentation by the investigator(s) who first reported a particular PAH in MSS. References of articles and/or presentations on specific PAHs that contained evidence later criticized are included plus references to the misinterpretations or errors. The criticism by FIESER (104) in 1957 of the shortcomings of the evidence $(6,46-48,69)$ supposedly indicating the presence of $\mathrm{B}[a] \mathrm{P}$ in cigarette smoke has already been mentioned. Two other notable situations involved 1,2-dihydrobenz[j]aceanthrylene (cholanthrene) and dibenzo[def,p]chrysene (formerly named dibenzo[ $a, l]$ pyrene, initially 1,2,3,4-dibenzopyrene). These two PAH identifications, based solely on UV spectral data, were found to be incorrect. In their study, RODGMAN and COOK (329) incorrectly defined a PAH as 1,2-dihydrobenz $[j]$ aceanthrylene (cholanthrene). In the massive study by USDA personnel on the identification of MSS PAHs, 1,2-dihydrobenz[j]aceanthrylene was not among the several benzocyclopentanthracenes reported $(419,422)$. The other incorrectly characterized PAH was dibenzo[def, $p]$ chrysene. For its identification, not only RODGMAN and COOK (329) but also BONNET and NEUKOMM (33), LYONS and JOHNSON (230), LYONS (228), WYNDER and WRIGHT (519), and PYRIKI (286) relied on published UV spectral data purportedly those of synthetic dibenzo[def, $p]$ chrysene (dibenzo[ $[a, l]$ pyrene). However, in 1966, LAVIT-LAMY and BUU-HoÏ (213) determined that the published UV spectral data were not those of dibenzo[ $[a, l]$ pyrene but of the isomeric dibenz $[a, e]$ aceanthrylene (dibenzo[a,e]fluoranthene), generated during the supposed synthesis of dibenzo[ $[a, l]$ pyrene. The authentic dibenzo[def,p]chrysene (dibenzo[ $[a, l]$ pyrene) was identified in MSS in 1977 (419), but its MSS level was not reported.

Some authorities insist that the $\mathrm{B}[a] \mathrm{P}$ and 4-(methylnitrosoamino)-1-(3-pyridinyl)-1-butanone (NNK) in cigarette smoke are the major causes of lung cancer in cigarette smokers (HECHT, 145; HECHT and HOFFMANN, 146a; HOFFMANN and HECHT, 152; WORLD HEALTH ORGANIZATION, 475a) despite the following: 1) Neither B $[a]$ P nor any other PAH in CSC either individually or in combination with the other PAHs in CSC can explain more than a few percent of the biological response observed in skin painting with CSC (DRUCKREY, 90; ROE, 343, 344; WRIGHT and WYNDER, 478; WYNDER, 483; WYNDER and HOFFMANN, 490, 495, 514a, 514b, 519); 2) Neither B [a]P nor any other $\mathrm{PAH}$ in CSC either individually or in combination with the other PAHs and assorted promoters (phenols) in CSC can explain more than a few percent of the biological response observed in skin painting with CSC (508); 3) In general, the $\mathrm{N}$-nitrosamines in CSC are not tumorigenic to mouse skin but are organ-specific tumorigens (PREUSSMANN and STEWART, 282b) ${ }^{\mathrm{g}}$, a point stressed in numerous reviews issued between the mid-1960s and the late 1990s on $\mathrm{N}$ nitrosamines (RODGMAN, 321a) and recognized by HoFFMANN and HECHT [see p. 75 in (152)]; 4) NNK has never been shown to induce lung cancer in a laboratory animal by inhalation (152).

While the minor contribution of $\mathrm{B}[a] \mathrm{P}$ to the tumorigenicity of CSC to mouse skin has been recognized since the mid1950s $(518,519)$, its presence in CSC has elicited continued interest since that time. Examination of the references to various smoke components reveals an interesting fact about $\mathrm{B}[a] \mathrm{P}$ : When all the cigarette smoke components are tabulated with regard to similar selection of references across the board, very few tobacco smoke components exceed $\mathrm{B}[a] \mathrm{P}$ in the number of pertinent references available. Obviously, the smoke component discussed most in publications and presentations between the mid-1950s and 2005 was nicotine. Next was acetaldehyde, followed by $\mathrm{B}[a] \mathrm{P}$.

Another interesting fact about $\mathrm{B}[a] \mathrm{P}$ is that, despite its minimal contribution to mouse-skin tumorigenicity from CSC, almost every year since the mid-1950s there has been at least one publication on a new and/or improved method to quantitate the yield of $\mathrm{B}[a] \mathrm{P}$ in MSS. In 2004, CORESTA published its recommended method for the determination of $\mathrm{B}[a] \mathrm{P}$ in tobacco smoke (70a). Much emphasis has been placed on the determination of $\mathrm{B}[a] \mathrm{P}$ in the MSS from fewer and fewer cigarettes. Before the advent of all the newly introduced and subsequently improved spectral and chromatographic systems, estimations of individual PAHs required the CSC from many cigarettes. For example, in their studies on the effect of various treatments of tobacco on the PAHs in MSS, RoDGMAN and CoOK $(309,314,327,330,331)$ chemically analyzed the MSS from 3600 cigarettes for each control and treated sample. For the MSS PAH analyses in the 50 treated and control samples described in (314), more than 183,000 cigarettes were smoked, the condensate collected, and processed. Nowadays, only a few cigarettes are needed for similar analyses. To permit comparison of the chemical data with the biological findings of WYNDER et al. (488), the smoking procedure used by them was duplicated in the RODGMAN-COOK studies in the 1950s, i.e., the cigarettes on a manifold were machine smoked (35-mL puff volume, 2sec puff duration, 3 puffs/min) with a collection system that duplicated the one described by WYNDER et al. (488). This smoking regime differed from the usual $35-\mathrm{mL}$ puff volume, 2-sec puff duration, 1 puff/min described by BRADFORD et al. (36) in 1936 and used by most investigators in smoke studies after that date.

\footnotetext{
${ }^{g}$ Subsequent to the publication of the PREUSSMANN and STEWART review (282b), DEUTSCH-WENZEL et al. (80a) reported that in a skinpainting study with $N^{\prime}$-nitrosonornicotine (NNN), tumors were initiated at the site of application. The specific tumorigenic potency of NNN was estimated to be only $0.8 \%$ of that of $\mathrm{B}[a] \mathrm{P}$. However no dose response relationship was observed with NNN over a treatment range of 12.5 to $200 \mu \mathrm{g}$.
} 


\begin{tabular}{|c|c|c|c|c|}
\hline No. & Polycyclic Aromatic Hydrocarbon & & CAS RN & References ${ }^{a}$ \\
\hline \multicolumn{5}{|c|}{ BICYCLIC: Benzenoid structures } \\
\hline 1 & Naphthalene & {$\left[\mathrm{C}_{10} \mathrm{H}_{8}\right][128]$} & $91-20-3$ & $\begin{array}{l}8,13-15,20,29,30,32,33,50,57,65,71,72 a, \\
81,93,95,97,102,103,113,114,124,126-128, \\
135,137,138,140,150,155,159,180,183,185, \\
192,198,204,209,229,231,234 a, 237,255, \\
257,263,265,278,280,283,292,293,302,308, \\
309,315,317,319,320,323,324,326,327,329, \\
332,334,336,338,341,342,357,360,361,365, \\
370,371-378,382,387,392,393,395,398, \\
400-402,407,415-418,420-422,423,425,432, \\
440,451,452,477,500,504,515,520,527,530\end{array}$ \\
\hline 2 & Naphthalene, alkyl- & & & $323,370,372-377,500,504$ \\
\hline 3 & Naphthalene, diethyl- & {$\left[\mathrm{C}_{14} \mathrm{H}_{16}\right][184]$} & $31831-35-3$ & 7 \\
\hline 4 & Naphthalene, dihydro- & {$\left[\mathrm{C}_{10} \mathrm{H}_{10}\right][130]$} & $29828-28-2$ & $234 a, 475$ \\
\hline $5-7^{\mathrm{b}}$ & Naphthalene, dihydrodimethyl- & {$\left[\mathrm{C}_{12} \mathrm{H}_{14}\right][158]$} & $72692-88-7$ & $126,234 a, 263,392$ \\
\hline $8-14^{\mathrm{b}}$ & Naphthalene, dihydromethyl- & {$\left[\mathrm{C}_{11} \mathrm{H}_{12}\right][144]$} & $39292-53-0$ & $124,126,263,392$ \\
\hline 15 & Naphthalene, 1,2-dihydro-3-methyl- & {$\left[\mathrm{C}_{11} \mathrm{H}_{12}\right][144]$} & $2717-44-4$ & $234 a, 475$ \\
\hline 16 & Naphthalene, 1,2-dihydro-4-methyl- & {$\left[\mathrm{C}_{11} \mathrm{H}_{12}\right][144]$} & $4373-13-1$ & $128,317,323,329,332,338$ \\
\hline 17 & $\begin{array}{l}\text { Naphthalene, 1,2-dihydro-5-methyl-3- } \\
\text { (1-methylethenyl)- }\end{array}$ & {$\left[\mathrm{C}_{14} \mathrm{H}_{16}\right][184]$} & $67494-22-8$ & 79,464 \\
\hline 18 & Naphthalene, 1,2-dihydro-1,1,6-trimethyl- & {$\left[\mathrm{C}_{13} \mathrm{H}_{16}\right][172]$} & $30364-38-6$ & $8,234 a, 342$ \\
\hline 19 & Naphthalene, 1,2-dihydro-1,5,8-trimethyl- & {$\left[\mathrm{C}_{13} \mathrm{H}_{16}\right][172]$} & $4506-36-9$ & $126,234 a, 264,392$ \\
\hline$A^{c}$ & Naphthalene, dimethyl- & {$\left[\mathrm{C}_{12} \mathrm{H}_{12}\right][156]$} & $28804-88-8$ & $124,126,135,234 a, 257,382,387,400$ \\
\hline 20 & Naphthalene, 1,2-dimethyl- & {$\left[\mathrm{C}_{12} \mathrm{H}_{12}\right][156]$} & 573-98-8 & $8,95,234 a, 263,265,398,416,420$ \\
\hline 21 & Naphthalene, 1,3-dimethyl- & {$\left[\mathrm{C}_{12} \mathrm{H}_{12}\right][156]$} & $575-41-7$ & $\begin{array}{l}8,95,234 a, 263,265,392,398,400-402,416, \\
420-422\end{array}$ \\
\hline 22 & Naphthalene, 1,4-dimethyl- & {$\left[\mathrm{C}_{12} \mathrm{H}_{12}\right][156]$} & $571-58-4$ & $8,95,234 a, 265,398,400-402,416,420-422$ \\
\hline 23 & Naphthalene, 1,5-dimethyl- & {$\left[\mathrm{C}_{12} \mathrm{H}_{12}\right][156]$} & $571-61-9$ & $8,95,234 a, 265,398,400-402,416,420-422$ \\
\hline 24 & Naphthalene, 1,6-dimethyl- & {$\left[\mathrm{C}_{12} \mathrm{H}_{12}\right][156]$} & $575-43-9$ & $\begin{array}{l}8,65,95,97,126,128,134,135,183,234 a, 255, \\
263,265,319,323,338,342,392,398,400-402, \\
416,420-422,425\end{array}$ \\
\hline 25 & Naphthalene, 1,7-dimethyl- & {$\left[\mathrm{C}_{12} \mathrm{H}_{12}\right][156]$} & $575-37-1$ & $8,95,234 a, 263,265,400-402,416,420-422$ \\
\hline 26 & Naphthalene, 1,8-dimethyl- & {$\left[\mathrm{C}_{12} \mathrm{H}_{12}\right][156]$} & $569-41-5$ & $\begin{array}{l}8,32,65,95,97,234 a, 265,278,323,338,342 \\
365,392,398,400-402,416,420-422,423,425\end{array}$ \\
\hline 27 & Naphthalene, 2,3-dimethyl- & {$\left[\mathrm{C}_{12} \mathrm{H}_{12}\right][156]$} & $581-40-8$ & $\begin{array}{l}8,95,234 a, 265,392,398,400-402,416 \\
420-422\end{array}$ \\
\hline 28 & Naphthalene, 2,6-dimethyl- & {$\left[\mathrm{C}_{12} \mathrm{H}_{12}\right][156]$} & $581-42-0$ & $\begin{array}{l}8,65,97,183,234 a, 255,323,338,342,392, \\
398,400-402,416,417,420-422,425\end{array}$ \\
\hline 29 & Naphthalene, 2,7-dimethyl- & {$\left[\mathrm{C}_{12} \mathrm{H}_{12}\right][156]$} & $582-16-1$ & $\begin{array}{l}8,65,95,97,183,234 a, 255,265,323,342,392 \\
400-402,416,420-422\end{array}$ \\
\hline 30 & Naphthalene, dimethyl-2-ethenyl- & {$\left[\mathrm{C}_{14} \mathrm{H}_{14}\right][182]$} & $71630-68-7$ & $234 a, 475$ \\
\hline 31 & Naphthalene, dimethylethyl- & {$\left[\mathrm{C}_{14} \mathrm{H}_{16}\right][184]$} & $65319-44-0$ & 234a, 392 \\
\hline 32 & Naphthalene, 1,4-dimethyl-2-ethyl- & {$\left[\mathrm{C}_{14} \mathrm{H}_{16}\right][184]$} & $71607-89-1$ & 264,392 \\
\hline 33 & Naphthalene, 1,4-dimethyl-5-ethyl- & {$\left[\mathrm{C}_{14} \mathrm{H}_{16}\right][184]$} & $66309-90-8$ & 416,420 \\
\hline 34 & $\begin{array}{l}\text { Naphthalene, 1,6-dimethyl-4-(1-methylethyl)- } \\
\text { 1,2,3,4-tetrahydro-, (1S-cis)- }\end{array}$ & {$\left[\mathrm{C}_{15} \mathrm{H}_{22}\right][202]$} & $483-77-2$ & 109,463 \\
\hline 35 & Naphthalene, dimethyl-2-phenyl- & {$\left[\mathrm{C}_{18} \mathrm{H}_{16}\right][232]$} & $71607-60-8$ & $234 a, 416,420$ \\
\hline 36 & Naphthalene, dimethyltetrahydro- & {$\left[\mathrm{C}_{12} \mathrm{H}_{16}\right][160]$} & & $126,263,392$ \\
\hline 37 & Naphthalene, dimethyl-1,2,3,4-tetrahydro- & {$\left[\mathrm{C}_{12} \mathrm{H}_{16}\right][160]$} & $\begin{array}{l}51855-29-9 \\
65338-07-0\end{array}$ & 234a, 264 \\
\hline 38 & Naphthalene, 1-ethenyl- & {$\left[\mathrm{C}_{12} \mathrm{H}_{10}\right][154]$} & $\begin{array}{r}826-74-4 \\
72843-02-8\end{array}$ & $8,234 a, 398,400-402,421,422$ \\
\hline 39 & Naphthalene, 2-ethenyl- & {$\left[\mathrm{C}_{12} \mathrm{H}_{10}\right][154]$} & $827-54-3$ & $8,234 a, 398,400-402,416,420-422$ \\
\hline $40-41^{b}$ & Naphthalene, 2-ethenylmethyl- & {$\left[\mathrm{C}_{13} \mathrm{H}_{12}\right][168]$} & $64031-89-6$ & $234 a, 397,416,420$ \\
\hline 42 & Naphthalene, 2-ethenyl-1-methyl- & {$\left[\mathrm{C}_{13} \mathrm{H}_{12}\right][168]$} & $35737-86-1$ & 475 \\
\hline$A^{d}$ & Naphthalene, ethyl- & {$\left[\mathrm{C}_{12} \mathrm{H}_{12}\right][156]$} & $27138-19-8$ & $124,237,361$ \\
\hline 43 & Naphthalene, 1-ethyl- & {$\left[\mathrm{C}_{12} \mathrm{H}_{12}\right][156]$} & $1127-76-0$ & $\begin{array}{l}8,95,175,234 a, 265,392,398,400-402,416 \\
420-422\end{array}$ \\
\hline 44 & Naphthalene, 2-ethyl- & {$\left[\mathrm{C}_{12} \mathrm{H}_{12}\right][156]$} & $939-27-5$ & $\begin{array}{l}8,95,175,234 a, 263,265,392,398,400-402, \\
416,420-422\end{array}$ \\
\hline 45 & Naphthalene, ethylmethyl- & {$\left[\mathrm{C}_{13} \mathrm{H}_{14}\right][170]$} & $31391-42-1$ & $8,95,234 a, 265,416,420$ \\
\hline 46 & Naphthalene, 1-ethyl-3-methyl- & {$\left[\mathrm{C}_{13} \mathrm{H}_{14}\right][170]$} & $17057-94-2$ & 95,265 \\
\hline 47 & Naphthalene, 1-ethyl-5-methyl- & {$\left[\mathrm{C}_{13} \mathrm{H}_{14}\right][170]$} & $17057-92-0$ & 95,265 \\
\hline 48 & Naphthalene, 1-ethyl-6-methyl- & {$\left[\mathrm{C}_{13} \mathrm{H}_{14}\right][170]$} & $31032-91-4$ & $95,234 a, 265$ \\
\hline
\end{tabular}




\begin{tabular}{ll}
\hline No. & Polycyclic Aromatic Hydrocarbon \\
\hline & \\
49 & Naphthalene, 1-ethyl-7-methyl- \\
50 & Naphthalene, 1-ethyl-8-methyl- \\
51 & Naphthalene, 2-ethyl-3-methyl- \\
52 & Naphthalene, 2-ethyl-4-methyl- \\
53 & Naphthalene, 2-ethyl-5-methyl- \\
54 & Naphthalene, 2-ethyl-6-methyl- \\
55 & Naphthalene, 2-ethyl-7-methyl- \\
56 & Naphthalene, hexamethyl- \\
$\mathrm{A}^{\mathrm{e}}$ & Naphthalene, methyl- \\
57 & Naphthalene, 1-methyl-
\end{tabular}

58

Naphthalene, 2-methyl-

Naphthalene, (1-methylethyl)Naphthalene, methylphenylNaphthalene, methyl-2-phenylNaphthalene, methylpropylNaphthalene, 1-(1-methylpropyl)Naphthalene, 1-(2-methylpropyl)Naphthalene, methyltetrahydroNaphthalene, methyl-1,2,3,4-tetrahydroNaphthalene, 1-methyl-1,2,3,4-tetrahydroNaphthalene, 2-methyl-1,2,3,4-tetrahydroNaphthalene, 5-methyl-1,2,3,4-tetrahydroNaphthalene, 6-methyl-1,2,3,4-tetrahydroNaphthalene, pentamethyl-

Naphthalene, 1-phenylNaphthalene, 2-phenyl-

Naphthalene, 2-phenyl-, monomethylNaphthalene, 2-phenyl-, monomethylNaphthalene, 1-propylNaphthalene, 2-propyl-

$9-81^{\text {b }} \quad$ Naphthalene, tetrahydrotrimethyl$82 \quad$ Naphthalene, 1,2,3,4-tetrahydro83 Naphthalene, 1,2,3,4-tetrahydrotrimethyl84 Naphthalene, 1,2,3,4-tetrahydro-

\section{5-87 ${ }^{\mathrm{b}} \quad$ Naphthalene, tetramethyl-}

$88-92^{\text {b }}$ Naphthalene, trimethyl-

$93^{\mathrm{g}} \quad$ Naphthalene, trimethyl-

$94 \quad$ Naphthalene, 1,2,4-trimethyl-

$95 \quad$ Naphthalene, 1,2,6-trimethyl-

$96 \quad$ Naphthalene, 1,2,7-trimethyl-

97 Naphthalene, 1,3,5-trimethyl-

98 Naphthalene, 1,3,6-trimethyl-

$99 \quad$ Naphthalene, 1,4,5-trimethyl$100 \quad$ Naphthalene, 1,4,6-trimethyl$101 \quad$ Naphthalene, 1,6,7-trimethyl102 Naphthalene, 2,3,6-trimethyl$103 \quad 1,1^{\prime}$-Binaphthylene<smiles>c1ccc2c(-c3cccc4ccccc34)cccc2c1</smiles>

CAS RN References ${ }^{a}$

BICYCLIC: Benzenoid structures (cont.)

$\left[\mathrm{C}_{13} \mathrm{H}_{14}\right][170] \quad 31032-92-5 \quad 95,234 \mathrm{a}, 265$

$\left[\mathrm{C}_{13} \mathrm{H}_{14}\right][170] \quad 61886-71-3 \quad 95,234 \mathrm{a}, 265$

$\left[\mathrm{C}_{13} \mathrm{H}_{14}\right][170] \quad 31032-94-7 \quad 95,234 \mathrm{a}, 265$

$\left[\mathrm{C}_{13} \mathrm{H}_{14}\right][170] \quad 17179-41-8 \quad 95,265$

$\left[\mathrm{C}_{13} \mathrm{H}_{14}\right][170] \quad 17059-53-9 \quad 95,265$

$\left[\mathrm{C}_{13} \mathrm{H}_{14}\right][170] \quad 7372-86-3 \quad 95,234 \mathrm{a}, 265$

$\left[\mathrm{C}_{13} \mathrm{H}_{14}\right][170] \quad 17059-55-1 \quad 95,126,234 \mathrm{a}, 264,265$

$\left[\mathrm{C}_{16} \mathrm{H}_{20}\right][212] \quad 77242-78-5 \quad 234 \mathrm{a}, 416,420$

$\left[\mathrm{C}_{11} \mathrm{H}_{10}\right][142] \quad 1321-94-4 \quad 234 \mathrm{a}, 237,377,378$

$\left[\mathrm{C}_{11} \mathrm{H}_{10}\right][142] \quad 90-12-0 \quad 8,13,65,97,124,126,128,134,135,155,180$, $183,192,234 a, 255,257,263,265,308,317$,

$319,323,329,332,334,336,338,341,342,361$, $382,387,392,393,398,400-402,407,415,416$, $420-423,425,452,453$

$\left[\mathrm{C}_{11} \mathrm{H}_{10}\right][142]$

91-57-6 8, 13, 65, 69, 97, 113, 114, 124, 126-128, 134 , $135,155,168,180,183,192,234 a, 255,257$, $263,265,278,317,319,323,329,332,334,336$, $338,341,342,361,365,382,387,392,393,398$, 400-402, 407, 415-418, 420-423, 425, 452, 453

$\left[\mathrm{C}_{13} \mathrm{H}_{14}\right][170] \quad 29253-36-9$ 8, 234a, 416, 420

$\left[\mathrm{C}_{17} \mathrm{H}_{14}\right][218] \quad 52991-56-7 \quad 142,234 \mathrm{a}$

$\left[\mathrm{C}_{17} \mathrm{H}_{14}\right][218] \quad 71607-61-9 \quad 234 a, 416,420$

$\left[\mathrm{C}_{14} \mathrm{H}_{16}\right][184] \quad 34540-66-4 \quad 7$

$\left[\mathrm{C}_{14} \mathrm{H}_{16}\right][184] \quad 1680-58-6 \quad 7,95,234 \mathrm{a}, 263,264$

$\left[\mathrm{C}_{14} \mathrm{H}_{16}\right][184] \quad 1669-91-6 \quad 95$

$\left[\mathrm{C}_{11} \mathrm{H}_{14}\right][146] \quad 71607-57-3 \quad 263,392$

$\left[\mathrm{C}_{11} \mathrm{H}_{14}\right][146] \quad 31291-71-1 \quad 168,234 \mathrm{a}$

$\left[\mathrm{C}_{11} \mathrm{H}_{14}\right][146] \quad 1559-81-5 \quad 475$

$\left[\mathrm{C}_{11} \mathrm{H}_{14}\right][146] \quad 3877-19-8 \quad 234 \mathrm{a}, 261$

$\left[\mathrm{C}_{11} \mathrm{H}_{14}\right][146] \quad 2809-64-5 \quad 7$

$\left[\mathrm{C}_{11} \mathrm{H}_{14}\right][146] \quad 1680-51-9 \quad 1,7,260,261,400-422$

$\left[\mathrm{C}_{15} \mathrm{H}_{18}\right][198] \quad 56908-81-7 \quad 8,234 \mathrm{a}, 416,420$

$\left[\mathrm{C}_{16} \mathrm{H}_{12}\right][204] \quad 605-02-7 \quad 1,234 \mathrm{a}, 332$

$\left[\mathrm{C}_{16} \mathrm{H}_{12}\right][204] \quad 612-94-222,234 \mathrm{a}, 317,323,329,334,338,388,416,420$, 439

$\left[\mathrm{C}_{17} \mathrm{H}_{14}\right][218] \quad 71697-04-6 \quad 475$

$\left[\mathrm{C}_{17} \mathrm{H}_{14}\right][218] \quad 27378-74-1 \quad 475$

$\left[\mathrm{C}_{13} \mathrm{H}_{14}\right][170] \quad 2765-18-6 \quad 95,234 \mathrm{a}$

$\left[\mathrm{C}_{13} \mathrm{H}_{14}\right][170] \quad 2027-19-2 \quad 95,234 \mathrm{a}$

$\left[\mathrm{C}_{13} \mathrm{H}_{18}\right][174]$ 121414-18-4 126, 392

$\left[\mathrm{C}_{10} \mathrm{H}_{12}\right][132] \quad 119-64-2 \quad 7,134,135,180,261,342$

$\left[\mathrm{C}_{13} \mathrm{H}_{18}\right][174] \quad 72843-02-8 \quad 475$

$\left[\mathrm{C}_{13} \mathrm{H}_{18}\right][174] \quad 475-03-6 \quad 8,94,234 \mathrm{a}, 263,342,392,407,425$

$\left[\mathrm{C}_{14} \mathrm{H}_{16}\right][184] \quad 28652-74-67,126,234 \mathrm{a}, 257,263,264,400,416,420-422$

$\left[\mathrm{C}_{13} \mathrm{H}_{14}\right][170] \quad 28652-77-97,95,124,126,257,263,264,382,387$, $400-402,416,420-422$

$\left[\mathrm{C}_{13} \mathrm{H}_{14}\right][170] \quad 26856-35-9$ 234a, 475

$\left[\mathrm{C}_{13} \mathrm{H}_{14}\right][170] \quad 2717-42-2 \quad 95,126,234 \mathrm{a}, 264,265,392$

$\left[\mathrm{C}_{13} \mathrm{H}_{14}\right][170] \quad 3031-05-8 \quad 95,265$

$\left[\mathrm{C}_{13} \mathrm{H}_{14}\right][170] \quad 486-34-0 \quad 95,265$

$\left[\mathrm{C}_{13} \mathrm{H}_{14}\right][170] \quad 2131-39-7 \quad 95,265$

$\left[\mathrm{C}_{13} \mathrm{H}_{14}\right][170] \quad 3031-08-1 \quad 65,95,97,126,183,234 \mathrm{a}, 255,265,323,338$, $342,392,425$

$\left[\mathrm{C}_{13} \mathrm{H}_{14}\right][170] \quad 2131-41-1 \quad 234 \mathrm{a}, 263,264,392$

$\left[\mathrm{C}_{13} \mathrm{H}_{14}\right][170] \quad 2131-42-2 \quad 95,265$

$\left[\mathrm{C}_{13} \mathrm{H}_{14}\right][170] \quad 2245-38-7 \quad 95,128,234 \mathrm{a}$

$\left[\mathrm{C}_{13} \mathrm{H}_{14}\right][170] \quad 829-26-5$ 95, 234a, 265, 417

$\left[\mathrm{C}_{20} \mathrm{H}_{14}\right][254] \quad 604-53-5 \quad 234 \mathrm{a}, 303,475$ 


\begin{tabular}{|c|c|c|c|c|}
\hline No. & Polycyclic Aromatic Hydrocarbon & & CAS RN & References $^{a}$ \\
\hline \multicolumn{5}{|c|}{ BICYCLIC: Benzenoid structures (cont.) } \\
\hline 104 & 1,1'-Binaphthylene, dimethyl- & {$\left[\mathrm{C}_{22} \mathrm{H}_{18}\right][282]$} & $71265-39-9$ & 216 \\
\hline 105 & 1,1'-Binaphthylene, ethyl- & {$\left[\mathrm{C}_{22} \mathrm{H}_{18}\right][282]$} & $71277-81-1$ & 216 \\
\hline 106 & 1,1'-Binaphthylene, methyl- & {$\left[\mathrm{C}_{21} \mathrm{H}_{16}\right][268]$} & $138497-96-8$ & $216,234 a$ \\
\hline 107 & 2,2'-Binaphthylene & {$\left[\mathrm{C}_{20} \mathrm{H}_{14}\right][254]$} & $612-78-2$ & $13,177,323,342$ \\
\hline 108 & 2,2'-Binaphthylene, dimethyl- & {$\left[\mathrm{C}_{22} \mathrm{H}_{18}\right][282]$} & $71294-43-4$ & 216 \\
\hline 109 & 2,2'-Binaphthylene, ethyl- & {$\left[\mathrm{C}_{22} \mathrm{H}_{18}\right][282]$} & $71277-82-2$ & 216 \\
\hline 110 & 2,2'-Binaphthylene, methyl- & {$\left[\mathrm{C}_{21} \mathrm{H}_{16}\right][268]$} & $41637-91-6$ & 216 \\
\hline
\end{tabular}

BICYCLIC: Cyclopentanoid-benzenoid structures

$111 \quad 1 H$-Indene<smiles>C1=Cc2ccccc2C1</smiles>

$1121 H$-Indene, diethyl-

$1131 \mathrm{H}$-Indene, 2,3-dihydro-

$1141 H$-Indene, 2,3-dihydrodimethyl-

$1151 \mathrm{H}$-Indene, 2,3-dihydro-1,2-dimethyl-

$116 \quad 1 H$-Indene, 2,3-dihydroethyl-

$117 \quad 1 \mathrm{H}$-Indene, 2,3-dihydromethyl-

$1181 H$-Indene, 2,3-dihydro-1-methyl-

$1191 \mathrm{H}$-Indene, 2,3-dihydro-2-methyl-

$1201 \mathrm{H}$-Indene, 2,3-dihydro-4-methyl-

$121 \quad 1 H$-Indene, 2,3-dihydro-5-methyl-

$1221 H$-Indene, 2,3-dihydrotrimethyl-

$123 \quad 1 H$-Indene, dimethyl-

$124 \quad 1 H$-Indene, dimethylethyl-

$1251 H$-Indene, 5,6-dimethyl-2-phenyl-

1261 1H-Indene, 2-(3',4'-dimethylphenyl)-

$127 \quad 1 H$-Indene, ethyl-

$128 \quad 1 H$-Indene, ethylmethyl-

$129 \quad 1 H$-Indene, ethylpentamethyl-

$130 \quad 1 H$-Indene, heptamethyl-

$131 \quad 1 H$-Indene, hexamethyl-

$132-134^{\text {b }} 1 H$-Indene, methyl-

$\begin{array}{ll}135 & 1 H \text {-Indene, 1-methyl- } \\ 136 & 1 H \text {-Indene, 2-methyl- } \\ 137 & 1 H \text {-Indene, 3-methyl- } \\ 138 & 1 H \text {-Indene, 1-methylene- } \\ 139 & 2 H \text {-Indene, 2-methylene- } \\ 140 & 1 H \text {-Indene, 4-methyl-2-(2'-methylphenyl)- } \\ 141 & 1 H \text {-Indene, 5-methyl-2-(4'-methylphenyl)- } \\ 142 & 1 H \text {-Indene, 6-methyl-2-(4'-methylphenyl)- } \\ 143 & 1 H \text {-Indene, 7-methyl-2-(2'-methylphenyl)- } \\ 144 & 1 H \text {-Indene, 7-(4'-methylphenyl)- } \\ 145 & 1 H \text {-Indene, pentamethyl- } \\ 146 & 1 H \text {-Indene, phenyl- } \\ 147 & 1 H \text {-Indene, 2-phenyl- } \\ 148 & 1 H \text {-Indene, phenyltrimethyl- } \\ 149-152^{b}{ }^{b} & 1 H \text {-Indene, tetramethyl- } \\ 153-155^{b} & 1 H \text {-Indene, trimethyl- } \\ 156 & \text { Azulene }\end{array}$

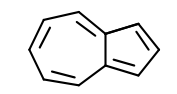

$\left[\mathrm{C}_{9} \mathrm{H}_{8}\right][116] \quad 95-13-68,13,15,65,120,121,126,134,135,140,237$, $240,257,263,323,336 a, 342,365,372-374$, $376,390,392,398,407,415,416,420-422,425$

\author{
$\left[\mathrm{C}_{13} \mathrm{H}_{16}\right][172] \quad 71278-05-2 \quad 416,420$ \\ $\left[\mathrm{C}_{9} \mathrm{H}_{10}\right][118] \quad 496-11-7 \quad 134,135,175,180,260,261,390,392$ \\ $\left[\mathrm{C}_{11} \mathrm{H}_{14}\right][146] \quad 53563-67-0 \quad 237,257,260,261,392,474$ \\ $\left[\mathrm{C}_{11} \mathrm{H}_{14}\right][146] \quad 17057-82-8 \quad 263,264,392$ \\ $\left[\mathrm{C}_{11} \mathrm{H}_{14}\right][146] \quad 71278-02-9 \quad 260,261,264,392$ \\ $\left[\mathrm{C}_{10} \mathrm{H}_{12}\right][132] \quad 27133-93-3 \quad 126,237,240,257,260,261,361$ \\ $\left[\mathrm{C}_{10} \mathrm{H}_{12}\right][132] \quad 767-58-8 \quad 126,168,261$ \\ $\left[\mathrm{C}_{10} \mathrm{H}_{12}\right][132] \quad 824-63-5 \quad 260,261$ \\ $\left[\mathrm{C}_{10} \mathrm{H}_{12}\right][132] \quad 824-22-6 \quad 180$ \\ $\left[\mathrm{C}_{10} \mathrm{H}_{12}\right][132] \quad 874-35-1 \quad 264,392$ \\ $\left[\mathrm{C}_{12} \mathrm{H}_{16}\right][160] \quad 36541-18-1 \quad 257,264,392$ \\ $\left[\mathrm{C}_{11} \mathrm{H}_{12}\right][144] \quad 29348-63-8 \quad 8,237,257,392,400-402,416,420-422$ \\ $\left[\mathrm{C}_{13} \mathrm{H}_{16}\right][172] \quad 71278-06-3 \quad 8,416,420$ \\ $\left[\mathrm{C}_{17} \mathrm{H}_{16}\right][220] \quad 22$ \\ $\left[\mathrm{C}_{17} \mathrm{H}_{16}\right][220] \quad 22$ \\ $\left[\mathrm{C}_{11} \mathrm{H}_{12}\right][144] \quad 58924-35-9 \quad 8,257,416,420$ \\ $\left[\mathrm{C}_{12} \mathrm{H}_{14}\right][158] \quad 77227-01-1 \quad 416,420$ \\ $\left[\mathrm{C}_{16} \mathrm{H}_{22}\right][214] \quad 71278-07-4 \quad 416,420$ \\ $\left[\mathrm{C}_{16} \mathrm{H}_{22}\right][214] \quad 77242-77-4 \quad 416,420$ \\ $\left[\mathrm{C}_{15} \mathrm{H}_{20}\right][200] \quad 71278-08-5 \quad 416,420$ \\ $\left[\mathrm{C}_{10} \mathrm{H}_{10}\right][130] \quad 29036-25-7 \quad 8,15,124,135,237,240,257,392,398,401$, \\ $402,416,420-422$ \\ $\left[\mathrm{C}_{10} \mathrm{H}_{10}\right][130] \quad 767-59-9 \quad 126,400-402,416,420-422$ \\ $\left[\mathrm{C}_{10} \mathrm{H}_{10}\right][130] \quad 2177-47-1 \quad 576,400-402,416,420$ \\ $\left[\mathrm{C}_{10} \mathrm{H}_{10}\right][130] \quad 767-60-2 \quad 263,392,400,416,420-422$ \\ $\left[\mathrm{C}_{10} \mathrm{H}_{8}\right][128] \quad 2471-84-3 \quad 392$ \\ $\left[\mathrm{C}_{10} \mathrm{H}_{8}\right][128] \quad 6596-86-7 \quad 392$ \\ $\left[\mathrm{C}_{17} \mathrm{H}_{16}\right][220] \quad 22$ \\ $\left[\mathrm{C}_{17} \mathrm{H}_{16}\right][220]$ \\ $\left[\mathrm{C}_{17} \mathrm{H}_{16}\right][220] \quad 22$ \\ $\left[\mathrm{C}_{17} \mathrm{H}_{16}\right][220] \quad 22$ \\ $\left[\mathrm{C}_{16} \mathrm{H}_{14}\right][206]$ \\ $\left[\mathrm{C}_{14} \mathrm{H}_{18}\right][186] \quad 71278-09-6 \quad 416,420$ \\ $\left[\mathrm{C}_{15} \mathrm{H}_{12}\right][192] \quad 38638-41-4 \quad 166,416,420$ \\ $\left[\mathrm{C}_{15} \mathrm{H}_{12}\right][192] \quad 4505-48-022$ \\ $\left[\mathrm{C}_{18} \mathrm{H}_{18}\right][234] \quad 22$ \\ $\left[\mathrm{C}_{13} \mathrm{H}_{16}\right][172] \quad 27135-78-0 \quad 8,257,416,420$ \\ $\left[\mathrm{C}_{12} \mathrm{H}_{14}\right][158] \quad 60826-61-1 \quad 8,237,257,400-402,416,420-422$ \\ $\left[\mathrm{C}_{10} \mathrm{H}_{8}\right][128] \quad 275-51-4 \quad 97,113,114,150,173,194,195,221,227,228$,
} $231,235,278,283,323,338,407,425$ 


\begin{tabular}{|c|c|c|c|c|}
\hline No. & Polycyclic Aromatic Hydrocarbon & & CAS RN & References $^{a}$ \\
\hline \multicolumn{5}{|c|}{ TRICYCLIC: Benzenoid structures } \\
\hline 157 & Anthracene & {$\left[\mathrm{C}_{14} \mathrm{H}_{10}\right][178]$} & $120-12-7$ & $\begin{array}{l}1,4,5,8,11,13,22,29,30,32-34,43,44,50, \\
51 a, 57,61,67-69,71,72 a, 74,76,81,93,96, \\
97,100,102,103,111,114,124,128,133 a, 135, \\
137,138,150,159,174,178,185,187,191,198, \\
209,219,221-223,225,227,231,236,239 a, \\
241,249,251-253,263,273,274,278,280,282, \\
283,287,289,302,308-310,315,317,319,320, \\
323,326,329-336,336 a, 338,341,342,345, \\
347,357,361,363-368,370,372-378,388,392, \\
395,398,400-402,417,418,421-425,433,434, \\
440,445-447,474,477,479,483,484,494,511, \\
515,517-520,524,526,529,530\end{array}$ \\
\hline 158 & Anthracene, alkyl- & & & $\begin{array}{l}69,100,229,273,278,323,342,372-374,376 \\
479,520\end{array}$ \\
\hline 159 & Anthracene, 9,10-dihydro- & {$\left[\mathrm{C}_{14} \mathrm{H}_{12}\right][180]$} & $613-31-0$ & $5,97,280,323,338,388,425$ \\
\hline $160-162^{b}$ & Anthracene, dimethyl- & {$\left[\mathrm{C}_{16} \mathrm{H}_{14}\right][206]$} & $29063-00-1$ & $368,388,400-402,416,420-422,440,530$ \\
\hline 163 & Anthracene, 9,10-dimethyl- & {$\left[\mathrm{C}_{16} \mathrm{H}_{14}\right][206]$} & $781-43-1$ & $30,336,353,388,529,530$ \\
\hline 164 & Anthracene, ethyl- & {$\left[\mathrm{C}_{16} \mathrm{H}_{14}\right][206]$} & $41637-86-9$ & 142,263 \\
\hline 165 & Anthracene, 9-ethyl- & {$\left[\mathrm{C}_{16} \mathrm{H}_{14}\right][206]$} & $605-83-4$ & 475 \\
\hline 166 & Anthracene, ethylmethyl- & {$\left[\mathrm{C}_{17} \mathrm{H}_{16}\right][220]$} & $71265-29-7$ & 216 \\
\hline 167 & Anthracene, 1-methyl- & {$\left[\mathrm{C}_{15} \mathrm{H}_{12}\right][192]$} & $610-48-0$ & $400-402,416,420-422,440$ \\
\hline 168 & Anthracene, 2-methyl- & {$\left[\mathrm{C}_{15} \mathrm{H}_{12}\right][192]$} & $613-12-7$ & $\begin{array}{l}22,29,69,97,138,278,323,338,342,365,392 \\
398,400-402,416,420-422,423-425,440\end{array}$ \\
\hline 169 & Anthracene, 9-methyl- & {$\left[\mathrm{C}_{15} \mathrm{H}_{12}\right][192]$} & $779-02-2$ & $\begin{array}{l}5,29,30,72 a, 138,302,317,323,329,332,334, \\
338,342,388,398,400-402,416,420-422, \\
423-425,440,530\end{array}$ \\
\hline 170 & Anthracene, propyl- & {$\left[\mathrm{C}_{17} \mathrm{H}_{16}\right][220]$} & $71265-30-0$ & 216 \\
\hline $171-172^{b}$ & Anthracene, trimethyl- & {$\left[\mathrm{C}_{17} \mathrm{H}_{16}\right][220]$} & $27358-28-7$ & $216,400-402,416,420-422,530$ \\
\hline 173 & Phenanthrene & {$\left[\mathrm{C}_{14} \mathrm{H}_{10}\right][178]$} & 85-01-8 & $\begin{array}{l}1,5,8,11,13,20,29,30,32,33,44,51 a, 57,65, \\
68,69,71,72 a, 81,96,97,100,102,103,113, \\
114,116,117,124,126,127,133 a, 135,138, \\
150,159,185,187,192,196,198,204,209,221, \\
231,238,239 a, 249,255,257,263,267,270, \\
274,278,280-283,285,288,289,292,293,302, \\
308-310,315,317,319,320,323,326,329,334, \\
336,336 a, 338,341,342,345,346,348,357, \\
361,364-366,368,370,372-378,388,392,398, \\
400-402,410,415,417,418,421-423,425,434, \\
440,474,477,479,484,494,515,520,529,530\end{array}$ \\
\hline 174 & Phenanthrene, alkyl- & & & 13, 51a, 100, 342, 372-374, 376, 479 \\
\hline 175 & Phenanthrene, dihydro- & {$\left[\mathrm{C}_{14} \mathrm{H}_{12}\right][180]$} & $26856-35-9$ & 198,342 \\
\hline 176 & Phenanthrene, 9,10-dihydro- & {$\left[\mathrm{C}_{14} \mathrm{H}_{14}\right][182]$} & $776-35-2$ & 126,342 \\
\hline 177 & Phenanthrene, dihydrodimethylene- & {$\left[\mathrm{C}_{16} \mathrm{H}_{12}\right][204]$} & $71607-56-2$ & 475 \\
\hline$A^{h}$ & Phenanthrene, dimethyl- & {$\left[\mathrm{C}_{16} \mathrm{H}_{14}\right][206]$} & $29062-98-4$ & $\begin{array}{l}32,51 a, 317,323,329,332,334,368,388 \\
400-402,419,421,422,529\end{array}$ \\
\hline 178 & Phenanthrene, 1,2-dimethyl- & {$\left[\mathrm{C}_{16} \mathrm{H}_{14}\right][206]$} & $20291-72-9$ & 21,128 \\
\hline 179 & Phenanthrene, 1,4-dimethyl- & {$\left[\mathrm{C}_{16} \mathrm{H}_{14}\right][206]$} & $22349-59-3$ & 174 \\
\hline 180 & Phenanthrene, 1,6-dimethyl- & {$\left[\mathrm{C}_{16} \mathrm{H}_{14}\right][206]$} & 20291-74-1 & 21 \\
\hline 181 & Phenanthrene, 1,7-dimethyl- & {$\left[\mathrm{C}_{16} \mathrm{H}_{14}\right][206]$} & $483-87-4$ & 21 \\
\hline 182 & Phenanthrene, 1,8-dimethyl- & {$\left[\mathrm{C}_{16} \mathrm{H}_{14}\right][206]$} & $7372-87-4$ & $1,182,278,323,338,342,425$ \\
\hline 183 & Phenanthrene, 2,5-dimethyl- & {$\left[\mathrm{C}_{16} \mathrm{H}_{14}\right][206]$} & $3674-66-6$ & 21,365 \\
\hline 184 & Phenanthrene, 2,6-dimethyl- & {$\left[\mathrm{C}_{16} \mathrm{H}_{14}\right][206]$} & $17980-16-4$ & 21 \\
\hline 185 & Phenanthrene, 2,7-dimethyl- & {$\left[\mathrm{C}_{16} \mathrm{H}_{14}\right][206]$} & $1576-69-8$ & $4,5,388$ \\
\hline 186 & Phenanthrene, 3,6-dimethyl- & {$\left[\mathrm{C}_{16} \mathrm{H}_{14}\right][206]$} & $1576-67-6$ & 196,338 \\
\hline 187 & Phenanthrene, 4,5-dimethyl- & {$\left[\mathrm{C}_{16} \mathrm{H}_{14}\right][206]$} & $3674-69-9$ & $419,421,422$ \\
\hline 188 & Phenanthrene, dimethylethyl- & {$\left[\mathrm{C}_{18} \mathrm{H}_{18}\right][234]$} & $71607-65-3$ & 475 \\
\hline 189 & Phenanthrene, ethyl- & {$\left[\mathrm{C}_{16} \mathrm{H}_{14}\right][206]$} & 30997-38-7 & 475 \\
\hline
\end{tabular}




\begin{tabular}{|c|c|c|c|c|}
\hline No. & Polycyclic Aromatic Hydrocarbon & & CAS RN & References $^{a}$ \\
\hline \multicolumn{5}{|c|}{ TRICYCLIC: Benzenoid structures (cont.) } \\
\hline 190 & Phenanthrene, ethylmethyl- & {$\left[\mathrm{C}_{17} \mathrm{H}_{16}\right][220]$} & $71607-66-4$ & 475 \\
\hline 191 & Phenanthrene, hexamethyl- & {$\left[\mathrm{C}_{20} \mathrm{H}_{22}\right][262]$} & $71607-67-5$ & 475 \\
\hline$A^{i}$ & Phenanthrene, methyl- & {$\left[\mathrm{C}_{15} \mathrm{H}_{12}\right][192]$} & $31711-53-2$ & $\begin{array}{l}8,128,198,323,342,361,368,377,378,388 \\
392,417,440,474\end{array}$ \\
\hline 192 & Phenanthrene, 1-methyl- & {$\left[\mathrm{C}_{15} \mathrm{H}_{12}\right][192]$} & $832-69-9$ & $\begin{array}{l}5,174,263,361,388,392,398,400-402,416, \\
420-422,425\end{array}$ \\
\hline 193 & Phenanthrene, 2-methyl- & {$\left[\mathrm{C}_{15} \mathrm{H}_{12}\right][192]$} & $2531-84-2$ & $5,263,392,398,400,416,420-422$ \\
\hline 194 & Phenanthrene, 3-methyl- & {$\left[\mathrm{C}_{15} \mathrm{H}_{12}\right][192]$} & $832-71-3$ & $4,5,263,388,398,400,416,420-422$ \\
\hline 195 & Phenanthrene, 4-methyl- & {$\left[\mathrm{C}_{15} \mathrm{H}_{12}\right][192]$} & $832-64-4$ & $21,398,400-402,421,422$ \\
\hline 196 & Phenanthrene, 9-methyl- & {$\left[\mathrm{C}_{15} \mathrm{H}_{12}\right][192]$} & $883-20-5$ & $\begin{array}{l}1,31,32,249,278,317,323,329,332,334,338 \\
365,398,400-402,416,420-422,425\end{array}$ \\
\hline 197 & Phenanthrene, 1-methyl-5-(1-methylethyl)- & {$\left[\mathrm{C}_{18} \mathrm{H}_{18}\right][234]$} & & 475 \\
\hline 198 & Phenanthrene, pentamethyl- & {$\left[\mathrm{C}_{19} \mathrm{H}_{20}\right][248]$} & $71607-68-6$ & $216,416,420$ \\
\hline 199 & Phenanthrene, propyl- & {$\left[\mathrm{C}_{17} \mathrm{H}_{16}\right][220]$} & $71607-69-7$ & 216 \\
\hline $200-204^{b}$ & Phenanthrene, tetramethyl- & {$\left[\mathrm{C}_{17} \mathrm{H}_{16}\right][234]$} & $71607-70-0$ & $336,416,420,529$ \\
\hline $205-207^{b}$ & Phenanthrene, trimethyl- & {$\left[\mathrm{C}_{17} \mathrm{H}_{16}\right][220]$} & $30232-26-9$ & $400-402,416,420-422$ \\
\hline
\end{tabular}

TRICYCLIC: Cyclopentanoid-benzenoid structures

\begin{abstract}
208 Acenaphthylene<smiles></smiles>

$\left[\mathrm{C}_{12} \mathrm{H}_{8}\right][152]$

208-96-8 3, 8, 13, 29, 32, 33, 43, 44, 67-70, 81, 97, 113, $114,124,126,127,134,135,137,150,155,174$, 185, 198, 209, 221-223, 227--229, 231, 257, $278,280,308,313,315,317,323,329,334,336$, $338,341,342,357,361,365,370,372-374,376$, $379,392,398,400-402,415,416,420-422,423$, $425,434,447,500,518,519,520,530$
\end{abstract}

209 Acenaphthylene, 1,2-dihydro-<smiles>c1cc2c3c(cccc3c1)CC2</smiles>

210 Acenaphthylene, 1,2-dihydrodimethyl-

211 Acenaphthylene, 1,2-dihydromethyl-

212 Acenaphthylene, 1,2-dihydrotetramethyl-

213-214 ${ }^{\mathrm{b}}$ Acenaphthylene, 1,2-dihydrotrimethyl-

215-216 ${ }^{\mathrm{b}}$ Acenaphthylene, dimethyl-

217 Acenaphthylene, 1,3-dimethyl-

218 Acenaphthylene, diphenyl-

219-220 ${ }^{\text {b }}$ Acenaphthylene, methyl-

221 Acenaphthylene, 1-methyl-

222 Acenaphthylene, 3-methyl-

223 Acenaphthylene, 4-methyl-

224 Acenaphthylene, 5-methyl-

225-226 ${ }^{\text {b }}$ Acenaphthylene, tetramethyl-

$227-228^{\text {b }}$ Acenaphthylene, trimethyl-

$229 \quad 1 H$-Benz[e]indene<smiles>C1=Cc2c(ccc3ccccc23)C1</smiles>

$230 \quad 1 H$-Benz[e]indene, dimethyl-

$231 \quad 1 H$-Benz[e]indene, methyl-

$2321 H$-Benz[f]indene

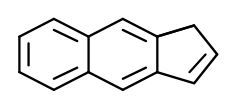

$\left[\mathrm{C}_{12} \mathrm{H}_{10}\right][154]$
83-32-9 8, 29, 32, 33, 50, 65, 72a, 97, 113, 114, 134, 135, $185,197,198,209,257,263,278,280,315,317$, $323,329,332,334,336,338,341,342,357,365$, $376,379,388,392,398,400-402,416,420-422$, $425,524,530$

$\left[\mathrm{C}_{14} \mathrm{H}_{14}\right][182]$

$\left[\mathrm{C}_{13} \mathrm{H}_{12}\right][168]$

$\left[\mathrm{C}_{16} \mathrm{H}_{18}\right][210]$

$\left[\mathrm{C}_{15} \mathrm{H}_{16}\right][196]$

$\left[\mathrm{C}_{14} \mathrm{H}_{12}\right][180]$

$\left[\mathrm{C}_{14} \mathrm{H}_{12}\right][180]$

$\left[\mathrm{C}_{24} \mathrm{H}_{16}\right][304]$

$\left[\mathrm{C}_{13} \mathrm{H}_{10}\right][166]$

$\left[\mathrm{C}_{13} \mathrm{H}_{10}\right][166]$

$\left[\mathrm{C}_{13} \mathrm{H}_{10}\right][166]$

$\left[\mathrm{C}_{13} \mathrm{H}_{10}\right][166]$

$\left[\mathrm{C}_{13} \mathrm{H}_{10}\right][166]$

$\left[\mathrm{C}_{16} \mathrm{H}_{16}\right][208]$

$\left[\mathrm{C}_{15} \mathrm{H}_{14}\right][194]$

$\left[\mathrm{C}_{13} \mathrm{H}_{10}\right][166]$

60684-29-9 400-402, 416, 420-422

36541-21-6 379, 400-402, 416, 420-422

60826-72-4 400-402, 416, 420-422

60826-69-9 400-402, 420-422

60826-68-8 8, 216, 400-402, 416, 420-422

19346-00-0 304

58546-40-6 216

58548-38-2 8, 257, 398, 400-402, 416, 420-422

19345-99-4 397, 400, 421, 422

19345-94-9 397, 399

$19345-97-299$

19345-91-6 99

60826-73-5 400-402, 416, 420-422

232-54-2 397
60826-70-2 8, 400-402, 416, 420-422
$\left[\mathrm{C}_{15} \mathrm{H}_{14}\right][194]$

$\left[\mathrm{C}_{14} \mathrm{H}_{12}\right][180]$

$\left[\mathrm{C}_{13} \mathrm{H}_{10}\right][166]$
416,420

64031-90-9 397

268-40-6 398, 400-402, 421, 422
$\left[\mathrm{C}_{15} \mathrm{H}_{14}\right][194]$

$\left[\mathrm{C}_{16} \mathrm{H}_{16}\right]$ [208]

$\left[\mathrm{C}_{14} \mathrm{H}_{12}\right][180]$

$\left[\mathrm{C}_{12} \mathrm{H}_{8}\right][152]$
60826-71-3 400-402, 416, 420-422

71265-34-4 416, 420

60826-63-3 400-402, 419, 421, 422

259-79-0 475

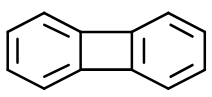




\begin{tabular}{|c|c|c|c|c|}
\hline No. & Polycyclic Aromatic Hydrocarbon & & CAS RN & References $^{a}$ \\
\hline \multicolumn{5}{|c|}{ TRICYCLIC: Cyclopentanoid-benzenoid structures (cont.) } \\
\hline 239 & $9 H$-Fluorene & {$\left[\mathrm{C}_{13} \mathrm{H}_{10}\right][166]$} & 86-73-7 & $\begin{array}{l}1,5,8,13,22,29,32,33,51 a, 57,72 a, 97,113, \\
114,124,126-128,135,138,157 a, 159,174, \\
185,198,238,239,239 a, 249,257,263,267, \\
278,280,282,294,302,317,323,324,329,332, \\
334,336,338,342,357,361,365,370,372-374, \\
376-378,383,388,391,392,398,400-402, \\
415-417,420-422,423,425,445,446,515,527 \\
530\end{array}$ \\
\hline $240-244^{b}$ & $9 H$-Fluorene, dimethyl- & {$\left[\mathrm{C}_{15} \mathrm{H}_{14}\right][194]$} & $30582-01-5$ & $22,294,392,400-402,416,420-422$ \\
\hline 245 & 9H-Fluorene, ?,9-dimethyl- & {$\left[\mathrm{C}_{15} \mathrm{H}_{14}\right][194]$} & & 416,420 \\
\hline 246 & $9 \mathrm{H}$-Fluorene, 1,9-dimethyl- & {$\left[\mathrm{C}_{15} \mathrm{H}_{14}\right][194]$} & $17057-98-6$ & $157 a, 263,295,392$ \\
\hline 247 & $9 \mathrm{H}$-Fluorene, 2,3-dimethyl- & {$\left[\mathrm{C}_{15} \mathrm{H}_{14}\right][194]$} & $4612-63-9$ & $157 a, 263,295$ \\
\hline 248 & 9H-Fluorene, 9,9-dimethyl- & {$\left[\mathrm{C}_{15} \mathrm{H}_{14}\right][194]$} & $4569-45-3$ & $157 a, 263$ \\
\hline 249 & $9 \mathrm{H}$-Fluorene, dimethylethyl- & {$\left[\mathrm{C}_{17} \mathrm{H}_{18}\right][222]$} & $71278-00-7$ & 416,420 \\
\hline $250-251^{b}$ & $9 \mathrm{H}$-Fluorene, ethyl- & {$\left[\mathrm{C}_{15} \mathrm{H}_{14}\right][194]$} & $65319-49-5$ & 416,420 \\
\hline 252 & $9 H$-Fluorene, 2-ethyl- & {$\left[\mathrm{C}_{15} \mathrm{H}_{14}\right][194]$} & $1207-20-1$ & $157 a, 263$ \\
\hline 253 & $9 H$-Fluorene, 9-ethyl- & {$\left[\mathrm{C}_{15} \mathrm{H}_{14}\right][194]$} & $2294-82-8$ & 416,420 \\
\hline $254-255^{b}$ & $9 H$-Fluorene, ethylmethyl- & {$\left[\mathrm{C}_{16} \mathrm{H}_{16}\right][208]$} & $71278-01-8$ & 416,420 \\
\hline$A^{j}$ & $9 \mathrm{H}$-Fluorene, methyl- & {$\left[\mathrm{C}_{14} \mathrm{H}_{12}\right][180]$} & $26914-17-0$ & $22,51 \mathrm{a}, 124,239,388$ \\
\hline 256 & $9 \mathrm{H}$-Fluorene, 1-methyl- & {$\left[\mathrm{C}_{14} \mathrm{H}_{12}\right][180]$} & $1730-37-6$ & $\begin{array}{l}5,8,22,97,157 a, 263,278,294,323,365,398 \\
400-402,416,420-422,425,455\end{array}$ \\
\hline 257 & $9 \mathrm{H}$-Fluorene, 2-methyl- & {$\left[\mathrm{C}_{14} \mathrm{H}_{12}\right][180]$} & $1430-97-3$ & $\begin{array}{l}8,22,126,128,157 a, 263,294,388,391,392 \\
398,400-402,416,420-422\end{array}$ \\
\hline 258 & 9H-Fluorene, 3-methyl- & {$\left[\mathrm{C}_{14} \mathrm{H}_{12}\right][180]$} & 2523-39-9 & $8,22,157 a, 294,392,400-402,416,420-422$ \\
\hline 259 & $9 \mathrm{H}$-Fluorene, 4-methyl- & {$\left[\mathrm{C}_{14} \mathrm{H}_{12}\right][180]$} & $1556-99-6$ & $\begin{array}{l}8,22,128,157 a, 263,294,365,392,400-402 \\
416,420-422\end{array}$ \\
\hline 260 & 9H-Fluorene, 9-methyl- & {$\left[\mathrm{C}_{14} \mathrm{H}_{12}\right][180]$} & $2523-37-7$ & $\begin{array}{l}8,22,32,97,157 a, 249,263,278,323,338,365 \\
398,401,402,416,420-422,425,455\end{array}$ \\
\hline 261 & 9H-Fluorene, 9-methylene- & {$\left[\mathrm{C}_{14} \mathrm{H}_{10}\right][178]$} & $4425-82-5$ & 159,295 \\
\hline 262 & $9 \mathrm{H}$-Fluorene, tetramethyl- & {$\left[\mathrm{C}_{17} \mathrm{H}_{18}\right][222]$} & $63372-50-9$ & 416,420 \\
\hline 263 & $9 H$-Fluorene, trimethyl- & {$\left[\mathrm{C}_{16} \mathrm{H}_{16}\right][208]$} & $30582-02-6$ & 416,420 \\
\hline
\end{tabular}

TETRACYCLIC: Benzenoid structures

$264 \quad$ Naphthacene

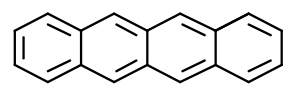

265 Benzanthracene, methyl-

266 Benz[a]anthracene<smiles>c1ccc2cc3c(ccc4ccccc43)cc2c1</smiles>

267 Benz[a]anthracene, alkyl268-269 ${ }^{\text {b }}$ Benz[a]anthracene, dimethyl270 Benz[a]anthracene, 7,12-dimethyl-

271 Benz[a]anthracene, ethyl272 Benz[a]anthracene, ethylmethyl273 Benz[a]anthracene, methyl274 Benz[a]anthracene, 1-methyl275 Benz[a]anthracene, 2-methyl276 Benz[a]anthracene, 3-methyl277 Benz[a]anthracene, 4-methyl-
$\left[\mathrm{C}_{18} \mathrm{H}_{12}\right][228]$

92-24-0 50, 65, 71, 97, 206, 239, 281, 302, 323, 425, 530

$\left[\mathrm{C}_{19} \mathrm{H}_{14}\right][242] \quad 63194-18-3 \quad 416,420$

$\left[\mathrm{C}_{18} \mathrm{H}_{12}\right][228] \quad 56-55-3 \quad 1,8,11,13,19,20,29,30,32-35,50,51 \mathrm{a}, 57$, $64,71,72 \mathrm{a}, 89,96,97,100,102,103,106,113$, $114,116-119,122,127,128,131,131 a, 133 a$, $138,152-157,160,162,163,165,167,174,182$, $185,187,191,192,198,204,219,222,223,227$, $230,238,239 a, 249,268,273,278,279,280$, $282,283,286,288,292,293,297,299-302$, $308-310,315,317,319,320,323,325,326$, $328-332,334,336,338,341,342,357,365,368$, $377,378,383,398,400-402,414,417,419-422$, 424-426, 432, 436, 439, 440, 441, 443, 444, $447-453,474,477,484,490,491,494,496,497$, $500,501,503,509,510,520,530$ 496

$\left[\mathrm{C}_{20} \mathrm{H}_{16}\right][256] \quad 43178-07-0 \quad 71,341,400-402,419-422,529,530$

$\left[\mathrm{C}_{20} \mathrm{H}_{16}\right][256] \quad 57-97-630,71,72 \mathrm{a}, 97,196,198,204,278,280,282$, $283,320,323,336,338,342,356,365,409-412$, $423,424,440,461,494,499,500,504,507,510$, 529,530

$\left[\mathrm{C}_{20} \mathrm{H}_{16}\right][256] \quad 31632-62-9 \quad 71,216,336,529$

$\left[\mathrm{C}_{21} \mathrm{H}_{18}\right][270] \quad 71265-32-2 \quad 475$

$\left[\mathrm{C}_{19} \mathrm{H}_{14}\right][242] \quad 43178-22-9239,398,401,402,419,421,422$

$\left[\mathrm{C}_{19} \mathrm{H}_{14}\right][242] \quad 2498-77-3 \quad 71,216,400 ; 419-422,530$

$\left[\mathrm{C}_{19} \mathrm{H}_{14}\right][242] \quad 2498-76-2 \quad 56,71,530$

$\left[\mathrm{C}_{19} \mathrm{H}_{14}\right][242] \quad 2498-75-1 \quad 71,97,216,278,530$

$\left[\mathrm{C}_{19} \mathrm{H}_{14}\right][242] \quad 316-49-4 \quad 71,216,530$ 


\begin{tabular}{|c|c|c|c|c|}
\hline No. & Polycyclic Aromatic Hydrocarbon & & CAS RN & References $^{a}$ \\
\hline \multicolumn{5}{|c|}{ TETRACYCLIC: Benzenoid structures (cont.) } \\
\hline 278 & Benz[a]anthracene, 5-methyl- & {$\left[\mathrm{C}_{19} \mathrm{H}_{14}\right][242]$} & $2319-96-2$ & $\begin{array}{l}32,71,97,249,286,317,323,329,332,334, \\
336,338,365,425,530\end{array}$ \\
\hline 279 & Benz[a]anthracene, 6-methyl- & {$\left[\mathrm{C}_{19} \mathrm{H}_{14}\right][242]$} & $316-14-3$ & 71,$216 ; 336,529$ \\
\hline 280 & Benz[a]anthracene, 8-methyl- & {$\left[\mathrm{C}_{19} \mathrm{H}_{14}\right][242]$} & $2381-31-9$ & 71,$216 ; 336,529,530$ \\
\hline 281 & Benz[a]anthracene, 9-methyl- & {$\left[\mathrm{C}_{19} \mathrm{H}_{14}\right][242]$} & $2381-16-0$ & $71,204,216,529,530$ \\
\hline 282 & Benz[a]anthracene, 10-methyl- & {$\left[\mathrm{C}_{19} \mathrm{H}_{14}\right][242]$} & $2381-15-9$ & $71,216,529,530$ \\
\hline 283 & Benz[a]anthracene, 12-methyl- & {$\left[\mathrm{C}_{19} \mathrm{H}_{14}\right][242]$} & $2422-79-9$ & $71,475,365,529,530$ \\
\hline 284 & Benz[a]anthracene, methylene- & {$\left[\mathrm{C}_{19} \mathrm{H}_{12}\right][240]$} & & 419 \\
\hline 285 & Benz[a]anthracene, propyl- & {$\left[\mathrm{C}_{21} \mathrm{H}_{18}\right][270]$} & 71265-33-3 & 216,529 \\
\hline 286 & Benz[a]anthracene, tetramethyl- & {$\left[\mathrm{C}_{22} \mathrm{H}_{20}\right][284]$} & & $71,416,420,529$ \\
\hline $287-288^{b}$ & Benz[a]anthracene, trimethyl- & {$\left[\mathrm{C}_{21} \mathrm{H}_{18}\right][270]$} & $60826-78-0$ & $71,216,336,400-402,416,420-422,529,530$ \\
\hline 289 & $7 H$-Benz[de]anthracene & {$\left[\mathrm{C}_{17} \mathrm{H}_{12}\right][216]$} & $199-94-0$ & $24 a$ \\
\hline
\end{tabular}<smiles>c1ccc2c(c1)Cc1cccc3cccc-2c13</smiles>

290 Benzo[c]phenanthrene<smiles>c1ccc2c(c1)ccc1ccc3ccccc3c12</smiles>

Benzo[c]phenanthrene, methylChrysene<smiles>c1ccc2c(c1)ccc1c3ccccc3ccc21</smiles>

Chrysene, alkyl-

Chrysene, dimethyl-

Chrysene, ethyl-

Chrysene, ethylmethyl-

Chrysene, methyl-

Chrysene, 1-methyl-

Chrysene, 2-methyl-

Chrysene, 3-methyl-

Chrysene, 4-methyl-

Chrysene, 5-methyl-

Chrysene, 6-methyl-

Chrysene, pentamethyl-

Chrysene, propyl-

Chrysene, tetramethyl-

Chrysene, trimethyl-

Triphenylene<smiles>c1ccc2c(c1)c1ccccc1c1ccccc21</smiles>

$\left[\mathrm{C}_{18} \mathrm{H}_{12}\right][228]$

195-19-7 37, 71, 97, 127, 133a, 150, 231, 165, 174, 278, $283,317,320,323,329,332,334,336,338,365$, $388,416,419,420,423,424,445,446,448-456$, $459,474,500,509,510,520,529,530$

$\left[\mathrm{C}_{19} \mathrm{H}_{14}\right][242] \quad 78328-47-9 \quad 38,71,336,529,530$

$\left[\mathrm{C}_{18} \mathrm{H}_{12}\right]$ [228] 218-01-9 1, 8, 11, 13, 29, 30, 37, 51a, 57, 71, 81, 96, 97, $100,106,131,131 \mathrm{a}, 133 \mathrm{a}, 137,138,141,146$, $150,152,153,156,160,162,163,165,167,174$, $185,187,191,198,201,204,209,230,231,238$, $268,273,278,280,282,283,286,300,302$, 308-311. 314, 315, 317, 319, 320, 323, 324, 326, $327,329-334,336,338,342,357,358,365,368$, $370,377,378,383,388,396,398,400-402,417$, $419,421-426,434,438-440,448-453,459,461$, $467,474,477,479,484,490,491,496,498,500$, $502,503,519,520,529,530$

$11,13,100,97,162,163,273,278,317,327$, $329,332,342,479,484,490-492,496,500$

$\left[\mathrm{C}_{20} \mathrm{H}_{16}\right][256] \quad 41637-92-7 \quad 1,11,97,278,283,286,323,338,365,400-402$, $419-422,425,529,530$

$\left[\mathrm{C}_{20} \mathrm{H}_{16}\right][256] \quad 71277-86-6 \quad 166,379$

$\left[\mathrm{C}_{21} \mathrm{H}_{18}\right][270] \quad 71277-87-7 \quad 216$

$\left[\mathrm{C}_{19} \mathrm{H}_{14}\right][242] \quad 41637-90-511,163,239,317,329,332,334,342,368,398$, 459,474

$\left[\mathrm{C}_{19} \mathrm{H}_{14}\right][242] \quad 3351-28-8 \quad 1,97,131,146,174,278,323,338,400-402$, 419-422, 425, 448-453, 459, 530

$\left[\mathrm{C}_{19} \mathrm{H}_{14}\right]$ [242] 3351-32-4 131, 146, 166, 174, 400-402, 419-422, 448-450, $451-453,529,530$

$\left[\mathrm{C}_{19} \mathrm{H}_{14}\right]$ [242] 3351-31-3 29, 131, 146, 166, 174, 400-402, 419-422,

$\begin{array}{ll} & 448-450,451-453,529,530 \\ 3351-30-2 & 166,174,400-402,419-422,529,530\end{array}$

$\left[\mathrm{C}_{19} \mathrm{H}_{14}\right][242]$

$\left[\mathrm{C}_{19} \mathrm{H}_{14}\right][242]$

$\left[\mathrm{C}_{19} \mathrm{H}_{14}\right][242]$

$\left[\mathrm{C}_{23} \mathrm{H}_{22}\right][298]$

$\left[\mathrm{C}_{21} \mathrm{H}_{18}\right][270]$

$\left[\mathrm{C}_{22} \mathrm{H}_{20}\right][284]$

$\left[\mathrm{C}_{21} \mathrm{H}_{18}\right][270]$

$\left[\mathrm{C}_{18} \mathrm{H}_{12}\right][228]$
3697-24-3 19, 106, 145, 146, 152-157, 160, 166, 167, 174, $268,319,320,322,326,336,357,400-402,414$, 414a, 448-453, 500, 509, 529, 530

1705-85-7 29, 131, 146, 166, 174, 400, 419-422, 448-453, 529,530

$\begin{array}{ll}71277-88-8 & 216 ; 416,420\end{array}$

$71277-89-9216$

$71277-90-2 \quad 416,420$

$60826-77-9$ 400-402, 416, 420-422

217-59-4 8, 71, 174, 131a, 133a, 150, 177, 231, 283, 323, $398,400-402,419,421,422,423,424,440,496$, 529,530 


\begin{tabular}{|c|c|c|c|c|}
\hline No. & Polycyclic Aromatic Hydrocarbon & & CAS RN & References $^{a}$ \\
\hline \multicolumn{5}{|c|}{ TETRACYCLIC: Benzenoid structures (cont.) } \\
\hline 308 & Triphenylene, dimethyl- & {$\left[\mathrm{C}_{20} \mathrm{H}_{16}\right][256]$} & $60826-76-8$ & $400-402,419-422$ \\
\hline 309 & Triphenylene, methyl- & {$\left[\mathrm{C}_{19} \mathrm{H}_{14}\right][242]$} & $41637-89-2$ & $400-402,419,421,422,530$ \\
\hline 310 & Triphenylene, trimethyl- & {$\left[\mathrm{C}_{21} \mathrm{H}_{18}\right][270]$} & $60826-79-1$ & $400-402,421,422$ \\
\hline 311 & Pyrene & {$\left[\mathrm{C}_{16} \mathrm{H}_{10}\right][202]$} & $129-00-0$ & $\begin{array}{l}1,4,5,8,11,13,20,22,29,30,32-35,43,44-46, \\
51 a, 57,61,67-69,71,72 a, 76,81,93,96,97, \\
100,113,114,127,128,131,131 a, 133 a, 137, \\
138,141,150,155,162,163,174,185,187,192, \\
198,200,209,219-223,227,229-231,234 a, 236, \\
239 a, 241,246,249,251-253,271,273,274, \\
278-280,282,283,285,287-289,302,308-311, \\
314,315,317,319,320,323,326,327,329, \\
332-334,336,338,341,342,357,363-366,368, \\
370,372-374,376-378,388,396,398,400-402, \\
410,411,418,419,421-425,427,433,434,439, \\
440,445-456,459,467,474,477,479,484,490, \\
491,494,496,500,519,520,524,526,530\end{array}$ \\
\hline 312 & Pyrene, alkyl- & & & $\begin{array}{l}81,100,162,163,229,273,274,278,323,342 \\
419,421,422,434,479,484,490,491,496,500\end{array}$ \\
\hline 313 & Pyrene, 1-butyl- & {$\left[\mathrm{C}_{20} \mathrm{H}_{18}\right][258]$} & $35980-18-8$ & 353 \\
\hline 314 & Pyrene, 1-decyl- & {$\left[\mathrm{C}_{26} \mathrm{H}_{30}\right][342]$} & $55682-90-1$ & 353 \\
\hline 315 & Pyrene, dihydro- & {$\left[\mathrm{C}_{16} \mathrm{H}_{12}\right][204]$} & $28779-32-0$ & $198,200,234 a, 342$ \\
\hline $316-318^{b}$ & Pyrene, dimethyl- & {$\left[\mathrm{C}_{18} \mathrm{H}_{14}\right][230]$} & $30582-03-7$ & $166,234 a, 368,398,400-402,419-422$ \\
\hline 319 & Pyrene, 1,3-dimethyl- & {$\left[\mathrm{C}_{18} \mathrm{H}_{14}\right][230]$} & $64401-21-4$ & 475 \\
\hline 320 & Pyrene, dimethyl-3,4-dimethylene- & {$\left[\mathrm{C}_{20} \mathrm{H}_{16}\right][256]$} & & 419 \\
\hline 321 & Pyrene, 3,4-dimethylene- & {$\left[\mathrm{C}_{18} \mathrm{H}_{12}\right][228]$} & $25732-74-5$ & 234a, 397, 400-402, 419, 421, 422 \\
\hline 322 & Pyrene, 3,4-dimethylenemethyl- & {$\left[\mathrm{C}_{19} \mathrm{H}_{14}\right][242]$} & & 419 \\
\hline 323 & Pyrene, ethyl- & {$\left[\mathrm{C}_{18} \mathrm{H}_{14}\right][230]$} & $56142-12-2$ & $100,278,323$ \\
\hline 324 & Pyrene, 1-ethyl- & {$\left[\mathrm{C}_{18} \mathrm{H}_{14}\right][230]$} & $56142-12-2$ & $278,338,353$ \\
\hline 325 & Pyrene, ethylmethyl- & {$\left[\mathrm{C}_{19} \mathrm{H}_{16}\right][244]$} & $71607-74-4$ & 216 \\
\hline 326 & Pyrene, hexamethyl- & {$\left[\mathrm{C}_{22} \mathrm{H}_{22}\right][286]$} & $71607-75-5$ & $234 a, 416,420$ \\
\hline 327 & Pyrene, 1-hexyl- & {$\left[\mathrm{C}_{22} \mathrm{H}_{22}\right][286]$} & $72692-89-8$ & $234 a, 353$ \\
\hline$A^{\prime}$ & Pyrene, methyl- & {$\left[\mathrm{C}_{17} \mathrm{H}_{12}\right][216]$} & $27577-90-8$ & $\begin{array}{l}1,8,81,234 a, 287,317,323,329,332,342,377 \\
378,434,440,448-450,451-456,459,474\end{array}$ \\
\hline 328 & Pyrene, 1-methyl- & {$\left[\mathrm{C}_{17} \mathrm{H}_{12}\right][216]$} & $2381-21-7$ & $\begin{array}{l}32,69,97,113,114,128,138,234 a, 246,249,278 \\
302,308,309,317,323,329,332,334,338,342, \\
365,368,398,400-402,419,421-425,461,530\end{array}$ \\
\hline 329 & Pyrene, 2-methyl- & {$\left[\mathrm{C}_{17} \mathrm{H}_{12}\right][216]$} & $3442-78-2$ & $\begin{array}{l}97,234 \mathrm{a}, 278,317,323,329,332,334,338,342, \\
365,368,398,400-402,419,421-425,455,459, \\
520,521,530\end{array}$ \\
\hline 330 & Pyrene, 4-methyl- & {$\left[\mathrm{C}_{17} \mathrm{H}_{12}\right][216]$} & $3353-12-6$ & $\begin{array}{l}32,81,97,128,138,141,163,234 a, 246,249 \\
278,280,317,323,329,332,334,338,342,365 \\
368,398,400-402,419,421-425,459,530\end{array}$ \\
\hline 331 & Pyrene, 1-octyl- & {$\left[\mathrm{C}_{24} \mathrm{H}_{26}\right][314]$} & $71608-00-9$ & 353 \\
\hline $332-334^{b}$ & Pyrene, pentamethyl- & {$\left[\mathrm{C}_{21} \mathrm{H}_{20}\right][272]$} & $71607-76-6$ & $234 a, 416,420$ \\
\hline 335 & Pyrene, propyl- & {$\left[\mathrm{C}_{19} \mathrm{H}_{16}\right][244]$} & $56142-09-7$ & 216 \\
\hline 336 & Pyrene, 1-tetradecyl- & {$\left[\mathrm{C}_{30} \mathrm{H}_{38}\right][398]$} & $71630-71-2$ & 353 \\
\hline 337 & Pyrene, tetrahydro- & {$\left[\mathrm{C}_{16} \mathrm{H}_{12}\right][206]$} & $66161-17-9$ & $200,201,342$ \\
\hline $338-341^{b}$ & Pyrene, tetramethyl- & {$\left[\mathrm{C}_{20} \mathrm{H}_{18}\right][258]$} & $60826-75-7$ & 234a, 400-402, 419-422 \\
\hline $342-344^{b}$ & Pyrene, trimethyl- & {$\left[\mathrm{C}_{19} \mathrm{H}_{16}\right][244]$} & $41637-88-1$ & $234 a, 216,400-402,419-422$ \\
\hline
\end{tabular}

TETRACYCLIC: Cyclopentanoid-benzenoid structures

345 Aceanthrylene $\quad\left[\mathrm{C}_{16} \mathrm{H}_{10}\right][202] \quad 202-03-9 \quad 400-402$<smiles>C1=Cc2cc3ccccc3c3cccc1c23</smiles> 


\begin{tabular}{|c|c|c|c|c|}
\hline No. & Polycyclic Aromatic Hydrocarbon & & CAS RN & References \\
\hline \multicolumn{5}{|c|}{ TETRACYCLIC: Cyclopentanoid-benzenoid structures (cont.) } \\
\hline 347 & Acephenanthrylene, 4,5-dihydro- & {$\left[\mathrm{C}_{16} \mathrm{H}_{12}\right][204]$} & $6232-48-0$ & 399,400 \\
\hline 348 & $5 H$-Benz $[f g]$ acenaphthylene & {$\left[\mathrm{C}_{15} \mathrm{H}_{10}\right][190]$} & $76774-50-0$ & 475 \\
\hline
\end{tabular}

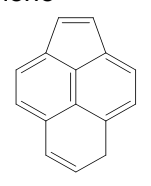

349 Benzofluorene, alkyl-

350-352 ${ }^{\mathrm{b}}$ Benzofluorene, dimethyl-

353-356 ${ }^{\text {b }}$ Benzofluorene, methyl-

357-358 ${ }^{\mathrm{b}}$ Benzofluorene, tetramethyl-

359 Benzofluorene, trimethyl-

360 Benzo[a]fluorene

$3615 H$-Benzo[a]fluorene

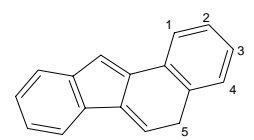

362

$11 \mathrm{H}$-Benzo[a]fluorene

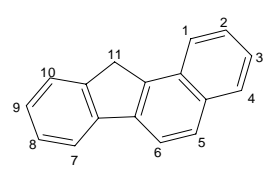

$363 \quad 11 H$-Benzo[a]fluorene, dimethyl-

$36411 H$-Benzo[a]fluorene, methyl-

$36511 H$-Benzo[a]fluorene, 11-methyl-

$366-368^{\mathrm{b}} 11 \mathrm{H}$-Benzo[a]fluorene, trimethyl-

$3695 \mathrm{H}$-Benzo[b]fluorene

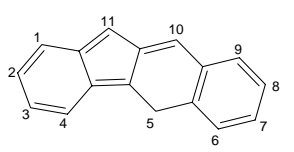

370

$11 \mathrm{H}$-Benzo[b]fluorene

$\left[\mathrm{C}_{17} \mathrm{H}_{12}\right][216]$

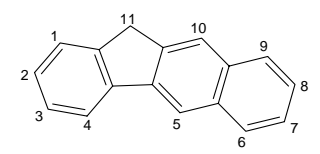

371

$11 \mathrm{H}$-Benzo[b]fluorene, methyl-

$11 \mathrm{H}$-Benzo[b]fluorene, 9-methyl-

$\left[\mathrm{C}_{18} \mathrm{H}_{14}\right][230]$

$\left[\mathrm{C}_{18} \mathrm{H}_{14}\right][230]$

$\begin{array}{ll}373 & \text { Benzo[C]fluorene, methyl- } \\ 374 & 7 H \text {-Benzo[c]fluorene }\end{array}$

$\left[\mathrm{C}_{18} \mathrm{H}_{14}\right][230]$

$\left[\mathrm{C}_{17} \mathrm{H}_{12}\right][216]$
$\left[\mathrm{C}_{19} \mathrm{H}_{16}\right][244]$

$\left[\mathrm{C}_{18} \mathrm{H}_{14}\right][230]$

$\left[\mathrm{C}_{18} \mathrm{H}_{14}\right][230]$

$\left[\mathrm{C}_{20} \mathrm{H}_{18}\right][258]$

$\left[\mathrm{C}_{17} \mathrm{H}_{12}\right][216]$
475

$\left[\mathrm{C}_{19} \mathrm{H}_{16}\right]$ [244] 7691-50-2 419-422

$\left[\mathrm{C}_{18} \mathrm{H}_{14}\right][230] \quad 60918-47-0 \quad 400-402,419-422$

$\left[\mathrm{C}_{21} \mathrm{H}_{20}\right][272] \quad 7691-51-3 \quad 416,420$

$\left[\mathrm{C}_{20} \mathrm{H}_{18}\right][258] \quad 401,402$

$\left[\mathrm{C}_{17} \mathrm{H}_{12}\right][216] \quad 30777-18-5 \quad 51 \mathrm{a}, 131,133 \mathrm{a}, 174,239 \mathrm{a}, 323,474$

$\left[\mathrm{C}_{17} \mathrm{H}_{12}\right][216] \quad 238-79-9 \quad 97,174,323,425$
238-84-6 1, 5, 11, 13, 32, 33, 97, 131, 133a, 174, 249, 278, $323,338,342,365,400-402,416,420-422,424$, $425,454-456,459,496,520$

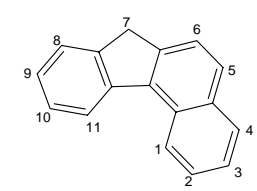

243-17-4 1, 5, 29, 97, 131, 133a, 163, 174, 278, 338, 342,

60826-64-4 11, 400-402, 419, 421, 422

$71265-25-3 \quad 1,11,32,97,249,278,323,338,342,425$

$71607-85-7 \quad 400,416,420$

243-18-5 97, 131, 133a, 174, 239a, 273, 278, 323, 365, 425 30777-19-6

\section{$243-17-4 \quad 1,5,29,97,131,133 a, 163,174,278,338,34$
$401,402,419,421,422,424,425,484,496$}

$60826-65-5 \quad 400-402,419,421,422$

419

416,420

205-12-9 97, 131, 133a, 174, 323, 338, 400-402, 419, 421, $422,425,520,530$ 


\begin{tabular}{l|l|l|l}
\hline No. & Polycyclic Aromatic Hydrocarbon & CAS RN & References $^{\text {a }}$ \\
\hline
\end{tabular}

TETRACYCLIC: Cyclopentanoid-benzenoid structures (cont.)

$376 \quad 11 \mathrm{H}$-Benzo[c]fluorene<smiles></smiles>

377

Cyclopenta[a]phenalene

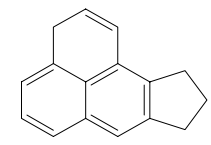

378-379 ${ }^{\mathrm{b}}$ Cyclopentaphenanthrene

$380-381^{\text {b }}$ Cyclopentaphenanthrene, methyl-

$382 \quad 15 \mathrm{H}$-Cyclopenta[a]phenanthrene<smiles>C1=Cc2ccc3c(ccc4ccccc43)c2C1</smiles>

15H-Cyclopenta[a]phenanthrene, 16,17-dihydro-

17H-Cyclopenta[a]phenanthrene

$\left[\mathrm{C}_{17} \mathrm{H}_{14}\right][218]$

$\left[\mathrm{C}_{17} \mathrm{H}_{12}\right][216]$<smiles>C1=Cc2c(ccc3c2ccc2ccccc23)C1</smiles>

385<smiles>c1ccc2c(c1)-c1cccc3cccc-2c13</smiles><smiles>c1cc2c3c(cccc3c1)CC2</smiles>

4H-Cyclopenta[def]phenanthrene, dimethyl-

$\left[\mathrm{C}_{17} \mathrm{H}_{14}\right][218]$<smiles></smiles>

$\left[\mathrm{C}_{20} \mathrm{H}_{18}\right]$ [258]

$\left[\mathrm{C}_{16} \mathrm{H}_{10}\right][202]$
$\left[\mathrm{C}_{17} \mathrm{H}_{14}\right][218] \quad 80455-52-3 \quad 398,400-402,416,420-422$

$\left[\mathrm{C}_{18} \mathrm{H}_{16}\right][232] \quad 81605-47-2 \quad 400-402,421,422$

$\left[\mathrm{C}_{17} \mathrm{H}_{12}\right][216] \quad 219-07-8 \quad 97,323,520$
$482-66-6 \quad 81,278,323,338,400,425,520$

219-08-9 167
$\left[\mathrm{C}_{19} \mathrm{H}_{16}\right][244] \quad 71277-92-4167$

$\left[\mathrm{C}_{18} \mathrm{H}_{14}\right][230] \quad 71277-93-5 \quad 167$

$\left[\mathrm{C}_{15} \mathrm{H}_{10}\right][190]$

$\left[\mathrm{C}_{17} \mathrm{H}_{14}\right][218] \quad 71277-91-3 \quad 475$

$\left[\mathrm{C}_{17} \mathrm{H}_{14}\right][218] \quad 65319-51-9 \quad 475$

$\left[\mathrm{C}_{16} \mathrm{H}_{12}\right][204] \quad 58548-39-3 \quad 475$

419

216

206-44-0 1, 4, 5, 8, 11, 13, 20, 21, 29, 30, 43, 44, 51a, 57. $65,67-69,72 a, 81,93,96,97,100,113,114$, $127,128,131,131 \mathrm{a}, 133 \mathrm{a}, 137,138,150,155$, 157b, 162, 163, 174, 182, 185, 187, 196, 209, 221-223, 230, 231, 239a, 255, 267, 273, 278, $280,282,289,294,302,308-311,314-317,319$ $320,323,326,329-334,336,338,341,342,357$, $365-368,370,372-374,376,383,388,396,398$, $400,410,411,419,421-425,427,434,439,440$, 448, 451-457, 459, 467, 477, 479, 484, 490, $491,494,496,500,502,503,519,520,524,530$ $13,97,100,157 \mathrm{~b}, 162,163,182,273,317,323$, $329,332,342,459,479,484,490,491,496,500$

$\left[\mathrm{C}_{16} \mathrm{H}_{12}\right][204] \quad 41593-24-2142$

$\left[\mathrm{C}_{19} \mathrm{H}_{14}\right][218] \quad 71278-25-6 \quad 142$ 


\begin{tabular}{|c|c|c|c|c|}
\hline No. & \multicolumn{2}{|l|}{ Polycyclic Aromatic Hydrocarbon } & CAS RN & References $^{a}$ \\
\hline \multicolumn{5}{|c|}{ TETRACYCLIC: Cyclopentanoid-benzenoid structures (cont.) } \\
\hline $397-399^{b}$ & ${ }^{b}$ Fluoranthene, dimethyl- & {$\left[\mathrm{C}_{18} \mathrm{H}_{14}\right][230]$} & $60826-74-6$ & $\begin{array}{l}278,298,323,365,368,398,400-402,419-422, \\
459\end{array}$ \\
\hline 400 & Fluoranthene, 8,9-dimethyl- & {$\left[\mathrm{C}_{18} \mathrm{H}_{14}\right][230]$} & $25889-63-8$ & $278,323,338,425,459$ \\
\hline 401 & Fluoranthene, ethyl- & {$\left[\mathrm{C}_{18} \mathrm{H}_{14}\right][230]$} & $55220-72-9$ & 263 \\
\hline 402 & Fluoranthene, ethylmethyl- & {$\left[\mathrm{C}_{19} \mathrm{H}_{16}\right][244]$} & $71277-96-8$ & 416,420 \\
\hline 403 & Fluoranthene, hexamethyl- & {$\left[\mathrm{C}_{22} \mathrm{H}_{22}\right][286]$} & $71277-97-9$ & 416,420 \\
\hline$A^{m}$ & Fluoranthene, methyl- & {$\left[\mathrm{C}_{17} \mathrm{H}_{12}\right][216]$} & $30997-39-8$ & $334,368,398,451-453$ \\
\hline 404 & Fluoranthene, 1-methyl- & {$\left[\mathrm{C}_{17} \mathrm{H}_{12}\right][216]$} & $25889-60-5$ & $263,400-402,419,421,422$ \\
\hline 405 & Fluoranthene, 2-methyl- & {$\left[\mathrm{C}_{17} \mathrm{H}_{12}\right][216]$} & $33543-31-6$ & $\begin{array}{l}174,263,400-402,419,421,422,448,451-453 \text {, } \\
530\end{array}$ \\
\hline 406 & Fluoranthene, 3-methyl- & {$\left[\mathrm{C}_{17} \mathrm{H}_{12}\right][216]$} & $1706-01-0$ & $174,263,448,451-453,530$ \\
\hline 407 & Fluoranthene, 7-methyl- & {$\left[\mathrm{C}_{17} \mathrm{H}_{12}\right][216]$} & $23339-05-1$ & 263,530 \\
\hline 408 & Fluoranthene, 8-methyl- & {$\left[\mathrm{C}_{17} \mathrm{H}_{12}\right][216]$} & $20485-57-8$ & $\begin{array}{l}97,278,323,338,400-402,419-422,425,454, \\
455,459,530\end{array}$ \\
\hline $409-410^{b}$ & ${ }^{b}$ Fluoranthene, pentamethyl- & {$\left[\mathrm{C}_{21} \mathrm{H}_{20}\right][272]$} & $71277-98-0$ & 416,420 \\
\hline 411 & Fluoranthene, propyl- & {$\left[\mathrm{C}_{19} \mathrm{H}_{16}\right][244]$} & $55220-69-4$ & 216 \\
\hline 412 & Fluoranthene, tetramethyl- & {$\left[\mathrm{C}_{20} \mathrm{H}_{18}\right][258]$} & $71277-99-1$ & 416,420 \\
\hline 413 & Fluoranthene, trimethyl- & {$\left[\mathrm{C}_{19} \mathrm{H}_{16}\right][244]$} & $41637-87-0$ & $400-402,416,420-422$ \\
\hline
\end{tabular}

PENTACYCLIC: Benzenoid structures

414 Benzo[b]chrysene $\quad\left[\mathrm{C}_{22} \mathrm{H}_{14}\right][278] \quad 214-17-5 \quad 13,150,231,278,283,338,530$<smiles></smiles>

$\left[\mathrm{C}_{22} \mathrm{H}_{14}\right][278] \quad 135-48-8 \quad 150,198,231,283,342,529,530$<smiles>c1ccc2cc3cc4cc5ccccc5cc4cc3cc2c1</smiles>

416 Benzo[a]naphthacene<smiles>c1ccc2cc3cc4c(ccc5ccccc54)cc3cc2c1</smiles>

417

418

Dibenzanthracene

Dibenz $[a, h]$ anthracene<smiles>c1ccc2c(c1)ccc1cc3c(ccc4ccccc43)cc12</smiles>

419 Dibenz[a,j]anthracene<smiles>c1ccc2c(c1)ccc1cc3ccc4ccccc4c3cc12</smiles>

420

Pentaphene<smiles>c1ccc2cc3c(ccc4cc5ccccc5cc43)cc2c1</smiles>

$\left[\mathrm{C}_{22} \mathrm{H}_{14}\right][278]$

226-88-0 97, 150, 231, 278, 283, 323, 338, 365, 425, 477, 519,530

$\left[\mathrm{C}_{22} \mathrm{H}_{14}\right][278]$
$\left[\mathrm{C}_{22} \mathrm{H}_{14}\right][278]$

$67775-07-9 \quad 475$

53-70-3 13, 20, 29, 30, 58, 71, 72a, 96, 97, 102, 103, 105a, 106, 122, 131, 131a, 133a, 137, 138, 145, $150,152-157,160,162,163,165,167,174,185$, $186,196,204,231,268,278,280,282,283,302$, $303,317,319,320,322-324,326,329,332,334$, $336,338,341,342,356,365,410,414,417,419$, 423-426, 432, 439, 445, 446, 448, 451-453, 458, $459,467,471,483,484,490,491,494,496,498$, $500,502,503,509,520,529,530$

$\left[\mathrm{C}_{22} \mathrm{H}_{14}\right][278]$

224-41-9 71, 131, 133a, 150, 174, 231, 283, 320, 357, 336, $419,423,424,529,530$

$\left[\mathrm{C}_{22} \mathrm{H}_{14}\right][278] \quad 222-93-5 \quad 71,97,278,283,323,483,519$ 


\begin{tabular}{|c|c|c|c|c|}
\hline No. & Polycyclic Aromatic Hydrocarbon & & CAS RN & References ${ }^{a}$ \\
\hline \multicolumn{5}{|c|}{ PENTACYCLIC: Benzenoid structures (cont.) } \\
\hline 421 & Perylene & {$\left[\mathrm{C}_{20} \mathrm{H}_{12}\right][252]$} & $198-55-0$ & $\begin{array}{l}\text { 5, 8, 13, 37, 64, 72a, 81, 96, 97, 113, 114, 127, } \\
\text { 131, 131a, 133a, 138, 150, 162, 163, 174, 187, } \\
\text { 198, 229-231, 239a, 278, 280, 282, 283, 302, 310, } \\
315,317,319,320,323,329,334,338,342,365, \\
398,400-402,419,421-424,434,439,459,467, \\
474,477,479,484,490,491,496,519,520,530\end{array}$ \\
\hline 422 & Perylene, alkyl- & & & 479 \\
\hline 423 & Perylene, dimethyl- & {$\left[\mathrm{C}_{22} \mathrm{H}_{16}\right][280]$} & $64760-19-6$ & 419 \\
\hline $424-425^{b}$ & Perylene, methyl- & {$\left[\mathrm{C}_{21} \mathrm{H}_{14}\right][266]$} & $64031-91-0$ & 419 \\
\hline 426 & Perylene, 3-methyl- & {$\left[\mathrm{C}_{21} \mathrm{H}_{14}\right][266]$} & $24471-47-4$ & 278 \\
\hline 427 & Picene & {$\left[\mathrm{C}_{22} \mathrm{H}_{14}\right][278]$} & $213-46-7$ & $13,198,283,341,342,419,423,424,530$ \\
\hline
\end{tabular}<smiles>c1ccc2c(c1)ccc1c2ccc2c3ccccc3ccc21</smiles>

428 Benzo[b]triphenylene<smiles>c1ccc2cc3c4ccccc4c4ccccc4c3cc2c1</smiles>

429 Benzo[b]triphenylene, methyl-

$\mathrm{A}^{\mathrm{n}} \quad$ Benzopyrene

430 Benzo[a]pyrene<smiles>c1ccc2c(c1)cc1ccc3cccc4ccc2c1c34</smiles>

$431 \quad$ Benzo[a]pyrene, alkyl-

432 Benzo[a]pyrene, 3,4-dihydro-

433 Benzo[a]pyrene, 7,8-dihydro-<smiles>C1=c2ccc3cc4ccccc4c4ccc(c2c34)CC1</smiles>

434-435 ${ }^{\mathrm{b}}$ Benzo[a]pyrene, dimethyl-

436 Benzo[a]pyrene, ethyl-

$437-438^{b}$ Benzo[a]pyrene, methyl-

439

3H-Benzo[cd]pyrene, 4,5-dihydro-<smiles>c1cc2ccc3ccc4c5c(cc(c1)c2c35)CCC4</smiles>

$\left[\mathrm{C}_{20} \mathrm{H}_{14}\right][254]$

$\left[\mathrm{C}_{20} \mathrm{H}_{14}\right][254]$

$\left[\mathrm{C}_{20} \mathrm{H}_{12}\right][252]$

$\left[\mathrm{C}_{20} \mathrm{H}_{12}\right]$ [252]

64760-20-9 419

$73467-76-2 \quad 324,529$

50-32-8 1-6, 8, 11-13, 16-20, 20a, 23-25, 27-35, 38,

40-49, 49a, 50, 51, 51a, 52-55, 57, 59-61, 64, 66, 68-70, 72a, 73-76, 78, 80, 81, 83-87, 89-93, 96, 97, $100,104-108,110,112-119,122,123,125-131$, 131a, 132, 133, 133a, 135a, 136-139, 141, 143, 145, $146,148-150,152-158,160-165,167,169-172$, 174, 179, 180a, 181, 184, 185, 187-193, 196-199, 203, 208-210, 214, 215, 217-227, 230, 231, 234, 234a, 236, 238, 239a, 241-246, 248, 249, 251-254, 258, 259, 262, 263, 265a, 265b, 266, 266a, 268, 269, 271-280, 282-284, 286-293, 295-297, 299-303, 305-311, 313-321, 323, 325-327, 329-334, 336-350, 356-359, 363-366, 368-370, 372-374, 376-378, 380, 383-386, 388, 392, 394, 395, 395a, 396-403, 405, 406, 410, 411, 414, 417-419, 421-425, 428-456, 459-461, 466-474, 475a, $476-486,490,492-496,498-507,511,512,514$, $515,515 a, 516-521,523,524,526-530$

$13,81,100,97,278,317,323,327,329,332$, $342,434,479,496,520$ $97,278,365,425$

17573-23-8 1, 234a, 278, 323, 338
$\left[\mathrm{C}_{22} \mathrm{H}_{16}\right][280] \quad 25167-90-2 \quad 59,234 \mathrm{a}, 323,400,419,421,422,530$
$\left[\mathrm{C}_{22} \mathrm{H}_{16}\right][280] \quad 71607-83-5 \quad 475$
$\left[\mathrm{C}_{21} \mathrm{H}_{14}\right][266] \quad 25167-89-9$ 234a, 278, 317, 323, 326, 329, 332, 334, 338, $377,378,398,400-402,419,421,422,425,459$, $500,509,530$
$\left[\mathrm{C}_{19} \mathrm{H}_{14}\right][242] \quad 7130-15-6 \quad 234 \mathrm{a}, 400,419,421,422$




\begin{tabular}{|c|c|c|c|c|}
\hline No. & Polycyclic Aromatic Hydrocarbon & & CAS RN & References $^{a}$ \\
\hline 440 & Benzo[e]pyrene & {$\left[\mathrm{C}_{20} \mathrm{H}_{12}\right][252]$} & $192-97-2$ & $\begin{array}{l}\text { 1, 5, 8, 13, 29, 30, 37, 51a, 64, 89, 93, 96, 97, } \\
100,131,133,133 a, 137-139,141,150,161, \\
162,174,185,187,209,219,227,231,234 a, \\
239 a, 262,271,273,278,280,282,283,286, \\
302,317,319,320,323,326,329,332,334,336, \\
338,342,356,365,368,377,378,388,394,396, \\
398,400-402,410,411,417,419,421-425,439, \\
440,448-455,459,460,467,484,490,491,496, \\
498,500,502,503,509,520,529,530\end{array}$ \\
\hline $441-442^{b}$ & Benzo[e]pyrene, dimethyl- & {$\left[\mathrm{C}_{22} \mathrm{H}_{16}\right][280]$} & $41699-06-3$ & 234a, 400, 419, 421, 422 \\
\hline $443-444^{b}$ & Benzo[e]pyrene, methyl- & {$\left[\mathrm{C}_{21} \mathrm{H}_{14}\right][266]$} & $41699-04-1$ & $234 a, 377,378,398,400-402,419,421,422,530$ \\
\hline 445 & Benzo[e]pyrene, trimethyl- & {$\left[\mathrm{C}_{23} \mathrm{H}_{18}\right][294]$} & $64760-21-0$ & $174,234 a, 419,448,451-453$ \\
\hline
\end{tabular}

PENTACYCLIC: Cyclopentanoid-benzenoid structures

446 Benz[a]aceanthrylene

$\left[\mathrm{C}_{20} \mathrm{H}_{12}\right][252] \quad$ 203-33-8 150, 231, 398, 400-402, 419, 421, 422

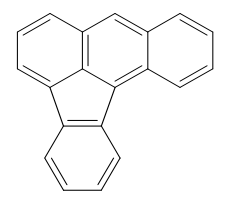

$447 \quad$ Benz[]]aceanthrylene

$\left[\mathrm{C}_{20} \mathrm{H}_{12}\right][252] \quad 202-33-5 \quad 475,530$

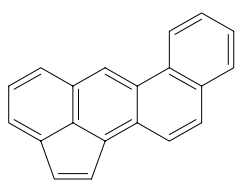

448

449

450

Benz[]]aceanthrylene, 1,2-dihydro-

$\left[\mathrm{C}_{20} \mathrm{H}_{14}\right][254]$

Benz[]]aceanthrylene, 1,2-dihydro-3-methyl-

$\left[\mathrm{C}_{21} \mathrm{H}_{16}\right][268]$

Benz[e]acephenanthrylene

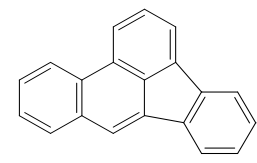

451

452

453

Benz[e]acephenanthrylene, methylBenz[e]acephenanthrylene, 10-methyl$1 H$-Benzo[a]cyclopent $h]$ anthracene, 2,3-dihydro-

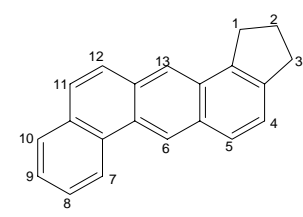

454

$9 H$-Benzo[a]cyclopent[i]anthracene, 10,11-dihydro-
$\left[\mathrm{C}_{20} \mathrm{H}_{12}\right][252]$

$\left[\mathrm{C}_{21} \mathrm{H}_{16}\right][266] \quad 41637-94-9$ 400-402, 419, 421, 422, 530

$\left[\mathrm{C}_{21} \mathrm{H}_{16}\right][266] \quad 149021-93-2 \quad 475$

$\left[\mathrm{C}_{21} \mathrm{H}_{16}\right][268] \quad 7099-43-6 \quad 31-35,37,71,97,230,249,250,278,286,317$, $323,329,334,338,341,365$

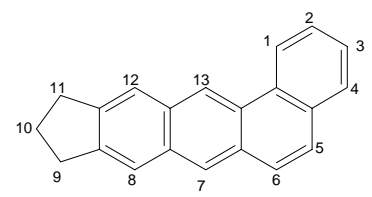

$\left[\mathrm{C}_{21} \mathrm{H}_{16}\right][268] \quad 7099-42-51,31-35,71,97,230,233,249,250,278,286$, $317,323,329,334,335,338,365,425,530$ 


\begin{tabular}{l|l|l|l}
\hline No. & Polycyclic Aromatic Hydrocarbon & CAS RN & References $^{\text {a }}$ \\
\hline
\end{tabular}

PENTACYCLIC: Cyclopentanoid-benzenoid structures (cont.)

455

4H-Cyclopenta[def]chrysene<smiles>c1ccc2c(c1)cc1c3c2ccc2cccc(c23)C1</smiles>

456

Cyclopenta[colfluoranthene

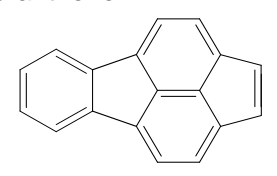

457

$1 \mathrm{H}$-Cyclopenta[a]pyrene

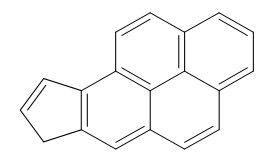

458

459

460

Cyclopenta[a]pyrene, 3,4-dihydro-

Cyclopenta[a]pyrene, 3,4-dihydromethylCyclopenta[cd]pyrene

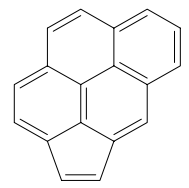

461

462

463

Cyclopenta[cd]pyrene, 3,4-dihydro-

Cyclopenta[cd]pyrene, 3,4-dihydromethyl$4 \mathrm{H}$-Cyclopenta[def]triphenylene<smiles></smiles>

$\left[\mathrm{C}_{19} \mathrm{H}_{12}\right][240]$

202-98-2 419, 530

$\left[\mathrm{C}_{18} \mathrm{H}_{10}\right][226]$

$193-54-4 \quad 475$

$\left[\mathrm{C}_{19} \mathrm{H}_{12}\right][240] \quad 42315-22-0 \quad 400-402,419$

$\left[\mathrm{C}_{19} \mathrm{H}_{14}\right]$ [242]

$\left[\mathrm{C}_{20} \mathrm{H}_{16}\right][256]$

$\left[\mathrm{C}_{18} \mathrm{H}_{10}\right][226] \quad 27208-37-3$ 131a, 133a, 234a, 419<smiles>c1cc2ccc3ccc4ccc5cccc(c2c1)c5c34</smiles>

Benzofluoranthene

Benzofluoranthene, dimethyl-

Benzofluoranthene, ethyl-

Benzofluoranthene, methyl-

$11 \mathrm{H}$-Benzo[cd]fluoranthene
Benzo[ghifluoranthene, dimethylBenzo[ghifluoranthene, ethylBenzo[ghifluoranthene, methylBenzo[ghifluoranthene, 1-methylBenzo[ghi]fluoranthene, 2-methylBenzo[ghi]fluoranthene, 3-methyl-
$\left[\mathrm{C}_{18} \mathrm{H}_{12}\right][228] \quad 25732-74-5475$

$\left[\mathrm{C}_{19} \mathrm{H}_{14}\right][242] \quad 64760-18-5 \quad 475$

$\left[\mathrm{C}_{19} \mathrm{H}_{12}\right][240] \quad 23992-32-7 \quad 419$<smiles>C1=CC2=CC=C3c4ccccc4-c4c3ccc(c42)C1</smiles>

$\left[\mathrm{C}_{18} \mathrm{H}_{10}\right][226]$

$\left[\mathrm{C}_{20} \mathrm{H}_{14}\right][254]$

$\left[\mathrm{C}_{20} \mathrm{H}_{14}\right][254]$

$\left[\mathrm{C}_{19} \mathrm{H}_{12}\right][240]$

$\left[\mathrm{C}_{19} \mathrm{H}_{12}\right][240]$
56832-73-6 5, 216, 417

416,420

216

416,420

16135-81-2 26, 182, 278, 323, 357, 365, 484, 518
$\left[\mathrm{C}_{18} \mathrm{H}_{10}\right][226]$ $323,329,332,334,338,342,357,365,368,398$, 400-402, 419, 421-425, 454, 455, 459, 461, 467, $474,484,490,491,496,497,500,510,520,530$

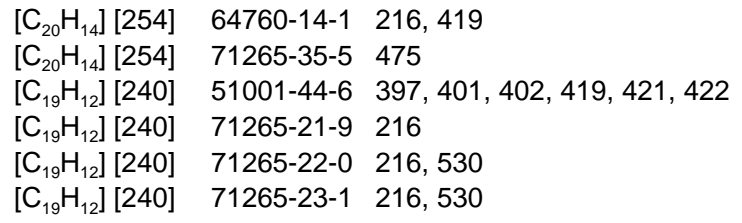




\begin{tabular}{|c|c|c|c|c|}
\hline No. & Polycyclic Aromatic Hydrocarbon & & CAS RN & References ${ }^{a}$ \\
\hline \multicolumn{5}{|c|}{ PENTACYCLIC: Cyclopentanoid-benzenoid structures (cont.) } \\
\hline 475 & Benzo[ghi]fluoranthene, 4-methyl- & {$\left[\mathrm{C}_{19} \mathrm{H}_{12}\right][240]$} & $71265-24-2$ & 475 \\
\hline 476 & Benzo[]fluoranthene & {$\left[\mathrm{C}_{20} \mathrm{H}_{12}\right][252]$} & $205-82-3$ & $\begin{array}{l}11,13,20,97,106,131,131 a, 133 a, 152-157, \\
160,162,163,165,167,174,278,317,319,320, \\
323,326,329,334,336,338,342,357,365,368, \\
414,414 a, 419,421-423,425,445,446,448, \\
451-453,459,467,474,484,490,491,496,497 \\
500,502,503,509,529,530\end{array}$ \\
\hline 477 & Benzo[]fluoranthene, methyl- & {$\left[\mathrm{C}_{21} \mathrm{H}_{14}\right][266]$} & $60826-67-7$ & $419,421,422$ \\
\hline 478 & Benzo[k]fluoranthene & {$\left[\mathrm{C}_{20} \mathrm{H}_{12}\right][252]$} & $207-08-9$ & $\begin{array}{l}4,13,20,29,37,51 a, 57,97,106,122,127,128, \\
131,131 a, 133 a, 137,150,152-157,160,162, \\
163,167,174,185,187,228,230,231,268,278, \\
317,319,320,323,326,329,332,334,336,338, \\
342,357,365,368,388,398,400-402,414, \\
414 a, 419,421-425,454,455,459,467,474, \\
484,490,491,496,497,500,510,530\end{array}$ \\
\hline 479 & Benzo[k]fluoranthene, methyl- & {$\left[\mathrm{C}_{21} \mathrm{H}_{14}\right][266]$} & $41637-93-8$ & $421,422,530$ \\
\hline 480 & 13H-Dibenzo[a,]fluorene & {$\left[\mathrm{C}_{21} \mathrm{H}_{14}\right][266]$} & $239-60-1$ & $\begin{array}{l}37,89,97,227,230,278,286,323,336,338 \\
342,425,530\end{array}$ \\
\hline
\end{tabular}<smiles>C1=CC2c3ccccc3CC2c2c1ccc1ccccc21</smiles>

$481 \quad 11 H$-Indeno[2,1-a]phenanthrene<smiles>c1ccc2c(c1)Cc1ccc3c(ccc4ccccc43)c1-2</smiles>

$\left[\mathrm{C}_{21} \mathrm{H}_{14}\right][266] \quad 220-97-3 \quad 34,97,278,323,338,342,351,353,425$

HEXACYCLIC: Benzenoid structures

482 Benzo[rst]pentaphene<smiles>c1ccc2c(c1)cc1ccc3cc4ccccc4c4ccc2c1c34</smiles>

483

484

\section{Benzoperylene}

Benzo[b]perylene<smiles>c1ccc2c(c1)c1cccc3c4cccc5cccc(c54)c2c3c1</smiles>

485
Benzo[ghi]perylene<smiles></smiles>

$\left[\mathrm{C}_{24} \mathrm{H}_{14}\right][302]$

$\left[\mathrm{C}_{24} \mathrm{H}_{14}\right][302]$

$\left[\mathrm{C}_{24} \mathrm{H}_{14}\right][302]$

11057-45-7 283, 475

197-70-6 419
$\left[\mathrm{C}_{22} \mathrm{H}_{12}\right][276]$
189-55-9 13, 20, 31, 32, 33-35, 62, 63, 97, 106, 112, 145, $150,152,153,155,160,165,174,231,247-250$ $268,283,286,317,319,320,322,323,326,329$, $332,334,336,338,342,356,358,365,389,414$, 414a, 419, 423-425, 445, 446, 448, 451-453, $483,500,509,520,529,530$

191-24-2 1, 8, 13, 29, 37, 57, 68, 69, 89, 93, 96, 97, 127, 131, 131a, 133a, 138, 137, 150, 162, 174, 185, $221,227,230,231,239 a, 273,278,280,286$, $302,308-310,315,319,320,323,329-331,332$, $334,336,338,342,357,365,398,419,423-425$, $448,451-453,455,459,462,467,483,484$, $490-492,496,500,510,520,529,530$ $486-488^{\text {b }}$ Benzo[ghi]perylene, dimethyl$489-490^{\mathrm{b}}$ Benzo[ghi]perylene, methyl491-492 ${ }^{\mathrm{b}}$ Benzo[ghi]perylene, trimethyl-
$\left[\mathrm{C}_{24} \mathrm{H}_{16}\right]$ [304] 64760-22-1 419

$\left[\mathrm{C}_{23} \mathrm{H}_{14}\right][290] \quad 41699-09-6 \quad 8,400,419$

$\left[\mathrm{C}_{25} \mathrm{H}_{18}\right][318] \quad 64760-23-2419$ 


$$
\begin{array}{l|l}
\hline \text { No. } & \text { Polycyclic Aromatic Hydrocarbon } \\
\hline 493 \quad \text { Dibenzo[b,def]chrysene }
\end{array}
$$

494 Dibenzo[def,mno]chrysene<smiles>c1cc2ccc3ccc4ccc5ccc(c6cc(c1)c26)c5c34</smiles>

495-496 b ${ }^{\text {b }}$ Dibenzo[def, mno]chrysene, dimethyl497-499 ${ }^{\mathrm{b}}$ Dibenzo[def,mno]chrysene, methyl-

500 Dibenzo[def,mno]chrysene, 6-methyl-

501 Dibenzo[def,p]chrysene<smiles></smiles>

502

Dibenzo[g,p]chrysene<smiles>c1ccc2c(c1)c1ccccc1c1c3ccccc3c3ccccc3c21</smiles>

503

Dibenzo[a,c]naphthacene<smiles>c1ccc2cc3cc4c5ccccc5c5ccccc5c4cc3cc2c1</smiles>

504

Dibenzo[a,j]naphthacene<smiles>c1ccc2c(c1)ccc1cc3cc4ccc5ccccc5c4cc3cc12</smiles>

505 Dibenzo[de,qr]naphthacene<smiles></smiles>

506

Dibenzo[fg,op]naphthacene

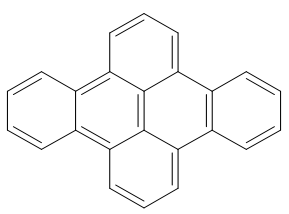

$\left[\mathrm{C}_{22} \mathrm{H}_{12}\right][276]$

$\left[\mathrm{C}_{24} \mathrm{H}_{16}\right]$ [304]

$\left[\mathrm{C}_{23} \mathrm{H}_{14}\right][290]$

$\left[\mathrm{C}_{23} \mathrm{H}_{14}\right][290]$

$\left[\mathrm{C}_{24} \mathrm{H}_{14}\right]$ [302]

64760-24-3 419

$41699-10-9 \quad 341,419$

460

191-30-0 35, 97, 100, 106, 145, 150, 152-157, 160, 165, $167,174,207,213,228,230,231,268,278,283$, $317,319,320,322,323,326,329,332,336,341$, $365,414,414 \mathrm{a}, 419,423,424,445,446,498$, $500,503,509,518,519,520,529,530$

$\left[\mathrm{C}_{26} \mathrm{H}_{16}\right][328] \quad 191-68-4 \quad 24 \mathrm{a}$
$\left[\mathrm{C}_{26} \mathrm{H}_{16}\right][328]$

$\left[\mathrm{C}_{24} \mathrm{H}_{14}\right][302]$

$\left[\mathrm{C}_{24} \mathrm{H}_{14}\right][302]$
227-04-3 97, 278, 323, 338, 425, 435, 477, 483, 490, 491, 519,520

193-09-9 150, 205, 231, 278, 317, 323, 329, 332,338 
Table 6 (cont.)

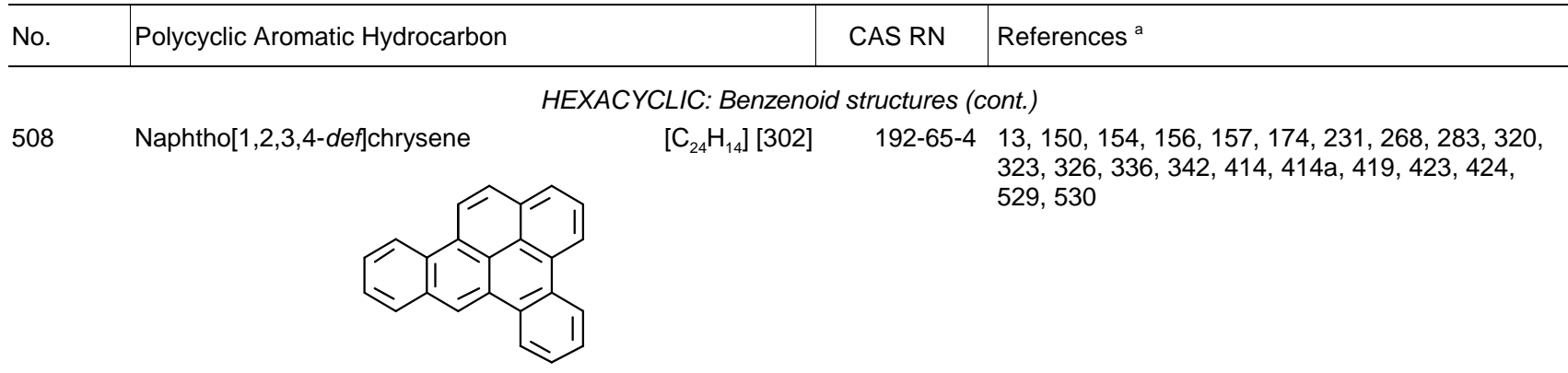

509 1H-Naphtho[3,2,1,8-defg]chrysene

$\left[\mathrm{C}_{23} \mathrm{H}_{14}\right][290] \quad 190-99-8 \quad 323,483$<smiles>C1=Cc2cc3cccc4c5ccccc5c5ccc(c2c3c45)C1</smiles>

$510 \quad$ Naphtho[2,1,8-qra]naphthacene

$\left[\mathrm{C}_{24} \mathrm{H}_{14}\right][302]$<smiles></smiles>

$511 \quad$ Naphtho[1,2-b]triphenylene<smiles></smiles>
$\begin{aligned} {\left[\mathrm{C}_{26} \mathrm{H}_{16}\right][328] \quad 215-26-9 } & \begin{array}{l}37,97,182,230,278,323,338,342,365,425, \\ 530\end{array}\end{aligned}$

HEXACYCLIC: Cyclopentanoid-benzenoid structures

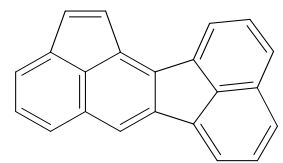

Acenaphth[1,2-a]acenaphthylene, methylCyclopenta[cd]perylene

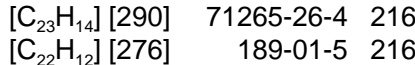

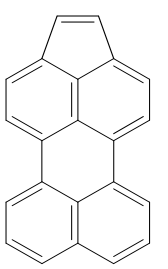

Cyclopenta[cd]perylene, methylDibenz[a,e]aceanthrylene

$\left[\mathrm{C}_{23} \mathrm{H}_{14}\right]$ [290]

$\left[\mathrm{C}_{24} \mathrm{H}_{14}\right][302]$<smiles></smiles>

216

5385-75-1 35, 150, 165, 174, 213, 228, 230, 231, 278, 286, $317,326,329,332,334,336,338,341,342,419$, $500,518,519,520,530$ 


\begin{tabular}{l|l|l|l}
\hline No. & Polycyclic Aromatic Hydrocarbon & CAS RN & References $^{\text {a }}$ \\
\hline
\end{tabular}

HEXACYCLIC: Cyclopentanoid-benzenoid structures (cont.)

517 Dibenz[j,mno]aceanthrylene<smiles></smiles>

518 Dibenz[j,mno]aceanthrylene, methyl-

$519-520^{\mathrm{b}}$ Dibenzofluoranthene

521 Dibenzo[j,]fluoranthene<smiles></smiles>

522

Indeno[1,2,3,4-defg]chrysene<smiles></smiles>

Indeno[1,2,3,4-defg]chrysene, methylIndeno[1,2,3-cd]fluoranthene<smiles>c1ccc2c(c1)-c1ccc3c4c(ccc-2c14)-c1ccccc1-3</smiles>

525

526

Indeno[1,2,3-cd]fluoranthene, methylIndeno[1,2,3-cd]pyrene<smiles></smiles>

527-528 ${ }^{\text {b }}$ Indeno[1,2,3-cd]pyrene, dimethyl$529-530^{\mathrm{b}}$ Indeno[1,2,3-cd]pyrene, methyl531 Indeno[1,2,3-cd]pyrene, trimethyl532 Naphth[1,2-e]acephenanthrylene
$\left[\mathrm{C}_{22} \mathrm{H}_{12}\right][276] \quad 71630-69-8216$

$\left[\mathrm{C}_{22} \mathrm{H}_{12}\right][276] \quad 668-30-4216$

$\left[\mathrm{C}_{23} \mathrm{H}_{14}\right][290] \quad 71277-94-6 \quad 216$

$\left[\mathrm{C}_{22} \mathrm{H}_{12}\right][276] \quad 193-43-1 \quad 11,13,93,97,106,133 \mathrm{a}, 137,152-157,160$ $163,165,167,174,268,323,326,414,419,425$, $448,451,452,496,500,509$

$\left[\mathrm{C}_{23} \mathrm{H}_{14}\right][290] \quad 41699-07-4 \quad 216$

$\left[\mathrm{C}_{22} \mathrm{H}_{12}\right][276] \quad 193-39-58,11,13,20,29,97,106,131,131 \mathrm{a}, 133 \mathrm{a}$, $152-157,160,163,165,167,174,185,234 a$, $268,319,320,323,326,336,338,342,357,398$, 414, 414a, 419, 421-422, 425, 448, 452, 453, $494,496,498,500,503,504,509,510,529,530$

$\left[\mathrm{C}_{23} \mathrm{H}_{14}\right][290] \quad 64158-99-2 \quad 234 \mathrm{a}, 419$

$\left[\mathrm{C}_{23} \mathrm{H}_{14}\right][290] \quad 64158-98-1 \quad 234 \mathrm{a}, 419$

$\left[\mathrm{C}_{25} \mathrm{H}_{18}\right][318] \quad 65140-04-7 \quad 419$

$\left[\mathrm{C}_{24} \mathrm{H}_{14}\right][302] \quad 5385-22-8 \quad 397,419$<smiles>C1=Cc2ccccc2C2=C3C(=Cc4ccccc43)C2=C1</smiles><smiles>c1cc2ccc3ccc4ccc5ccc6ccc1c1c2c3c4c5c61</smiles>

HEPTACYCLIC: Benzenoid structure $320,323,327,329,332,334,338,342,365,398$, $419,423-425,460,483,496,500,520,529,530$ 


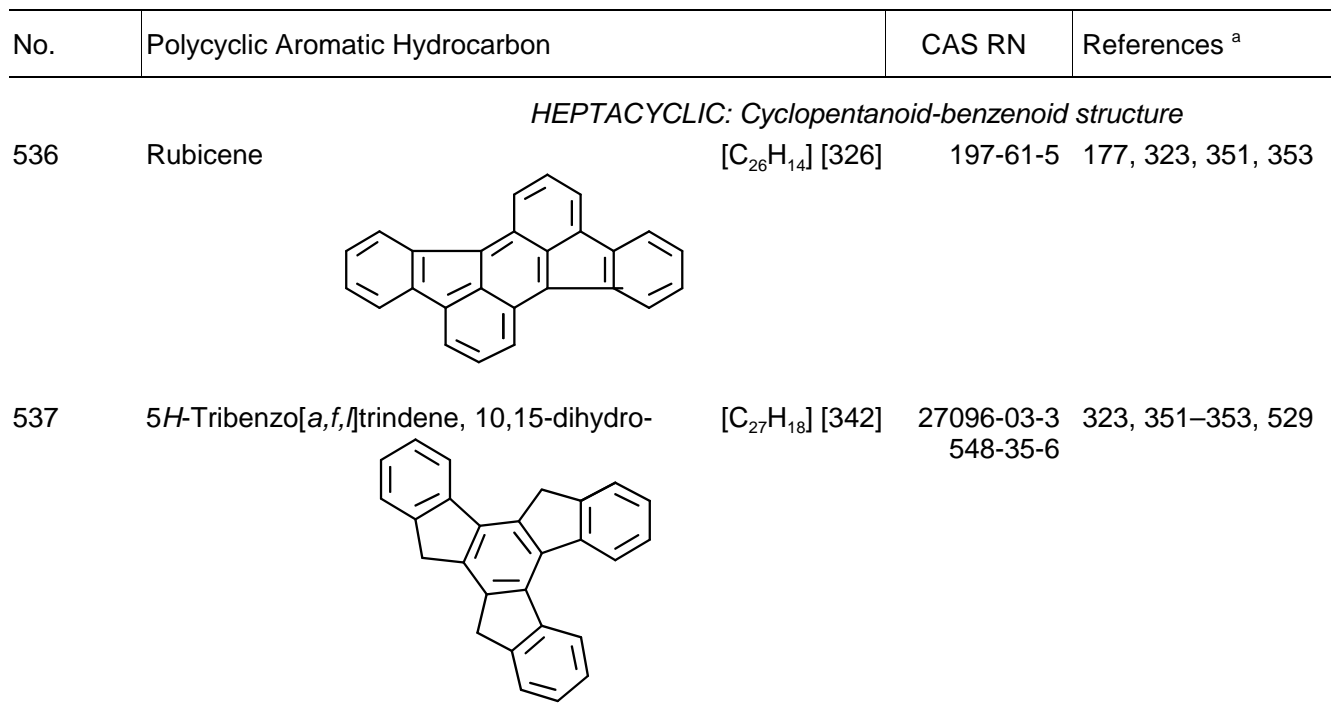

NONACYCLIC: Cyclopentanoid-benzenoid structure

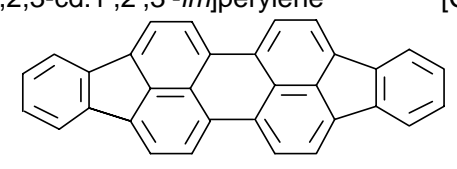

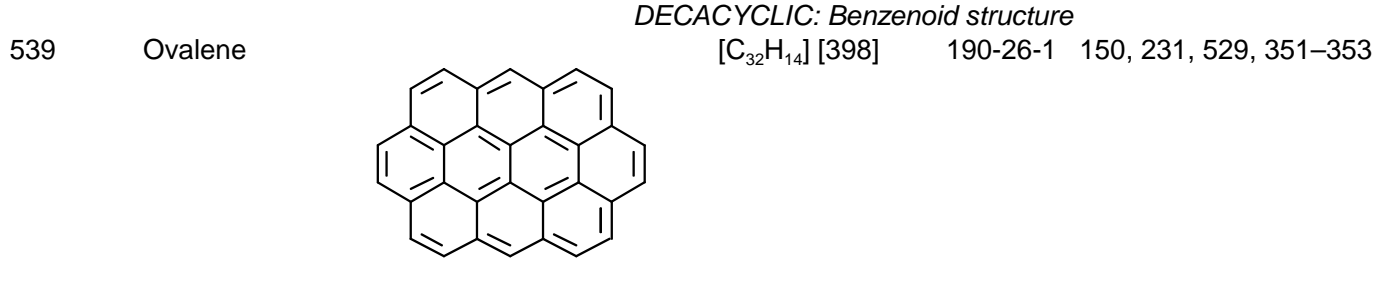

DECACYCLIC: Benzenoid structure

\footnotetext{
${ }^{a}$ Many of the citations contain additional references pertinent to the PAH in question.

${ }^{\mathrm{b}} \mathrm{A}$ numerical range indicates that more than one isomer of the PAH was reported.

${ }^{\circ}$ This PAH was not numbered because 1) the positions of the two methyl groups were not specified in the papers cited and 2) PAHs listed as 20 through 29 account for the dimethylnaphthalenes.

${ }^{\mathrm{d}}$ This PAH was not numbered because 1) the position of the ethyl group was not specified and it could have been either the 1-ethyl- or 2-ethylisomer or a mixture of them and 2) PAHs listed as 42 and 43 include both known ethylnaphthalenes.

${ }^{e}$ This PAH was not numbered because 1) the position of the methyl group was not specified and it could have been either the 1-methyl- or 2methyl- isomer or a mixture of them and 2) PAHs listed as 57 and 58 include both known methylnaphthalenes.

${ }^{\mathrm{f}}$ This PAH was not numbered because 1) the position of the methyl group was not specified in the paper cited and 2) it was uncertain whether two isomers were reported.

${ }^{9}$ The positions of the three methyl group were not specified. This isomer was different from five isomers previously reported by Snook et al. (421).

${ }^{\mathrm{h}}$ This PAH was not numbered because 1) the position of the dimethyl groups was not specified in the papers cited and 2) PAHs listed as 179 through 187 account for known dimethylphenanthrenes.

${ }^{i}$ This PAH was not numbered because 1) the position of the methyl group was not specified in the papers cited and 2) PAHs listed as 192 through 196 account for the five known methylphenanthrenes.

i This PAH was not numbered because 1) the position of the methyl group was not specified in the papers cited and 2) PAHs listed as 256 through 260 account for the five known methylfluorenes.

${ }^{k}$ This PAH was not numbered because 1) the position of the methyl group was not specified in the papers cited and 2) PAHs listed as 297 through 302 account for the five known methylchrysenes.

'This PAH was not numbered because 1) the position of the methyl group was not specified in the papers cited and 2) PAHs listed as 328 through 330 account for the three known methylpyrenes.

${ }^{m}$ This PAH was not numbered because 1) the position of the methyl group was not specified in the papers cited and 2) PAHs listed as 404 through 408 account for the five known methylfluoranthenes.

${ }^{n}$ This PAH was not numbered because in the papers cited, it was unclear whether it was $\mathrm{PAH} 430(\mathrm{~B}[a] \mathrm{P})$ or $\mathrm{PAH} 440(\mathrm{~B}[e] \mathrm{P})$ or a mixture thereof

- This PAH was not numbered because 1) the position of the methyl group was not specified in the papers cited and 2) PAHs listed as 472 through 475 account for the four known methylbenz[ghi]fluoranthenes.
}

Table 7 summarizes the PAHs identified in CSC that were included in earlier descriptions of proposed structure-tumorigenicity theories.

Examination of Table 7 indicates that most of the PAHs considered in the various theoretical systems designed to establish a relationship between molecular structure and tumorigenicity are totally benzenoid. Only a few PAHs with a combined benzenoid-cyclopentanoid structure were included in the early studies. LACASSAGNE et al. (204) in their discourse on structure-tumorigenicity relationship mentioned a few benzenoid PAHs but their major emphasis was on the structure-tumorigenicity relationship of numerous angular benzacridines. While the number of aza-arenes, including the benzacridines, in CSC is less than the number 


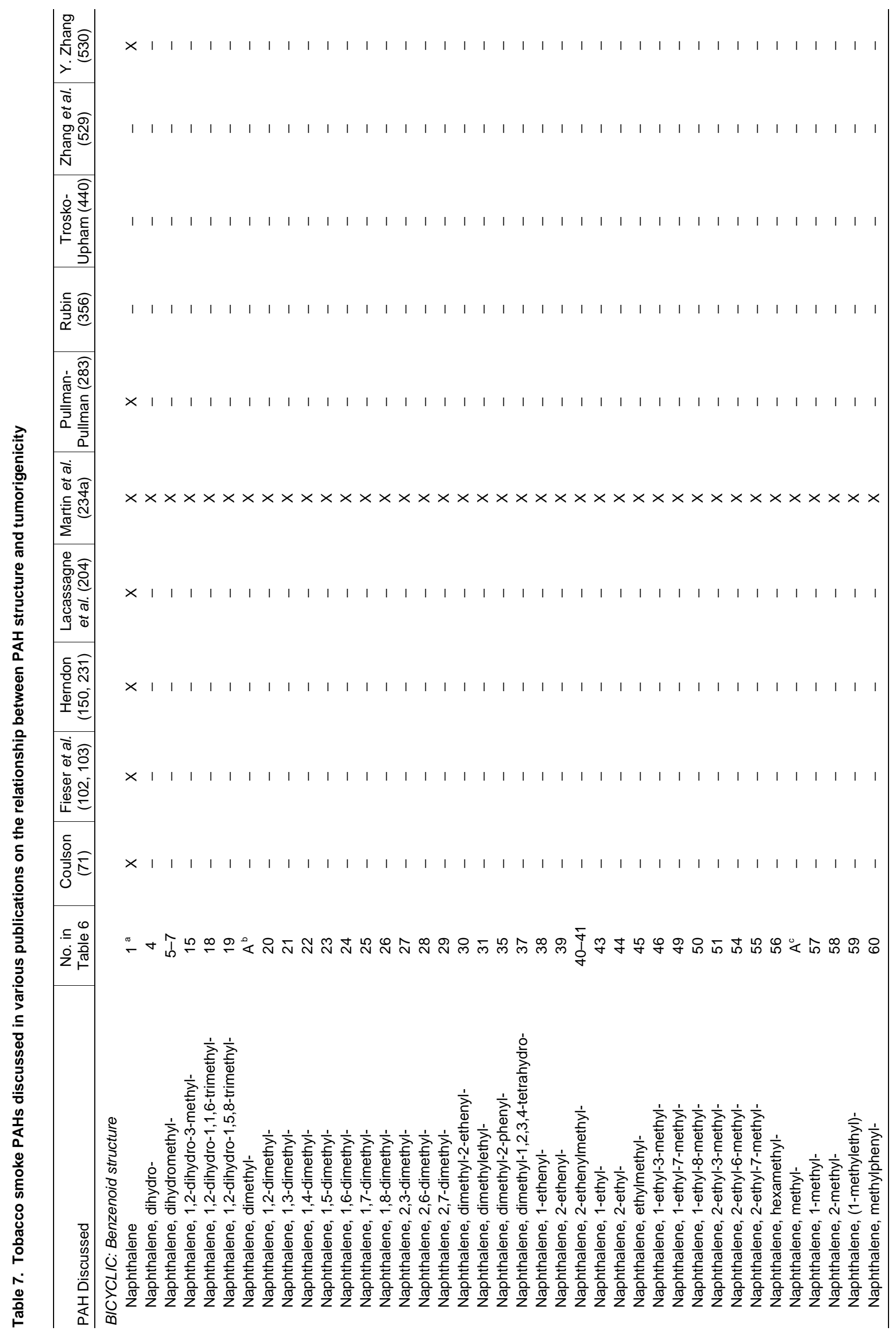




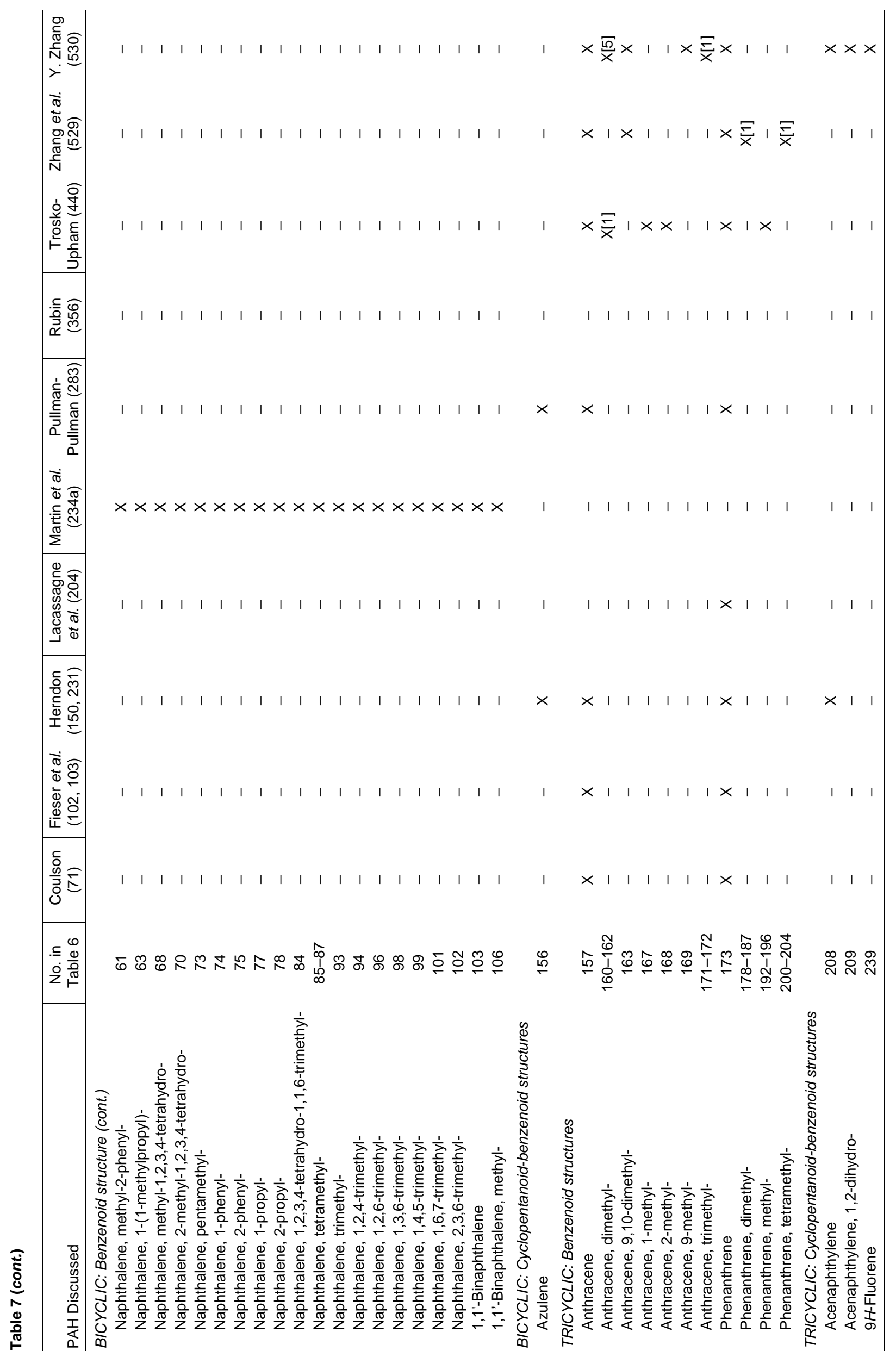




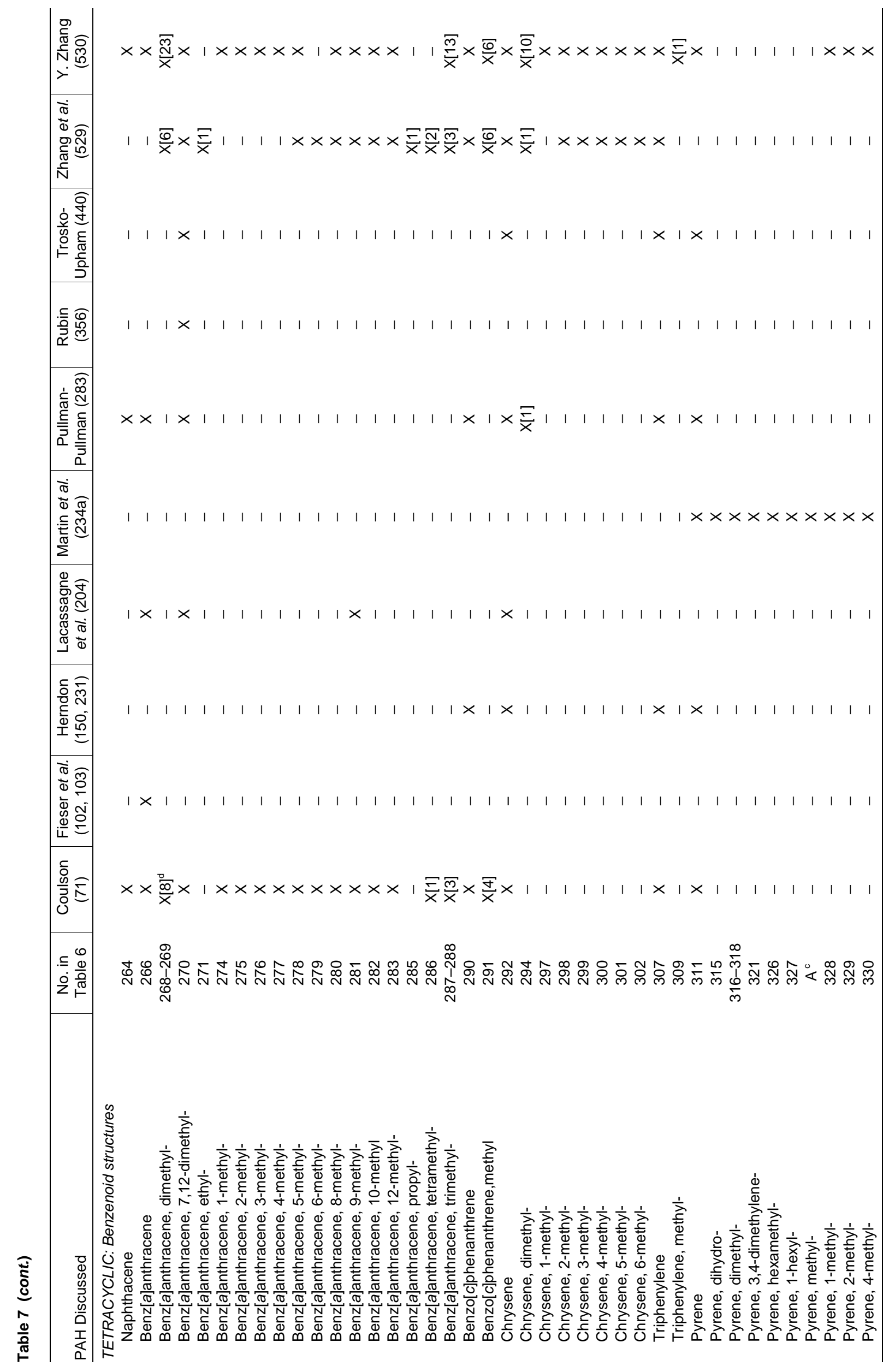




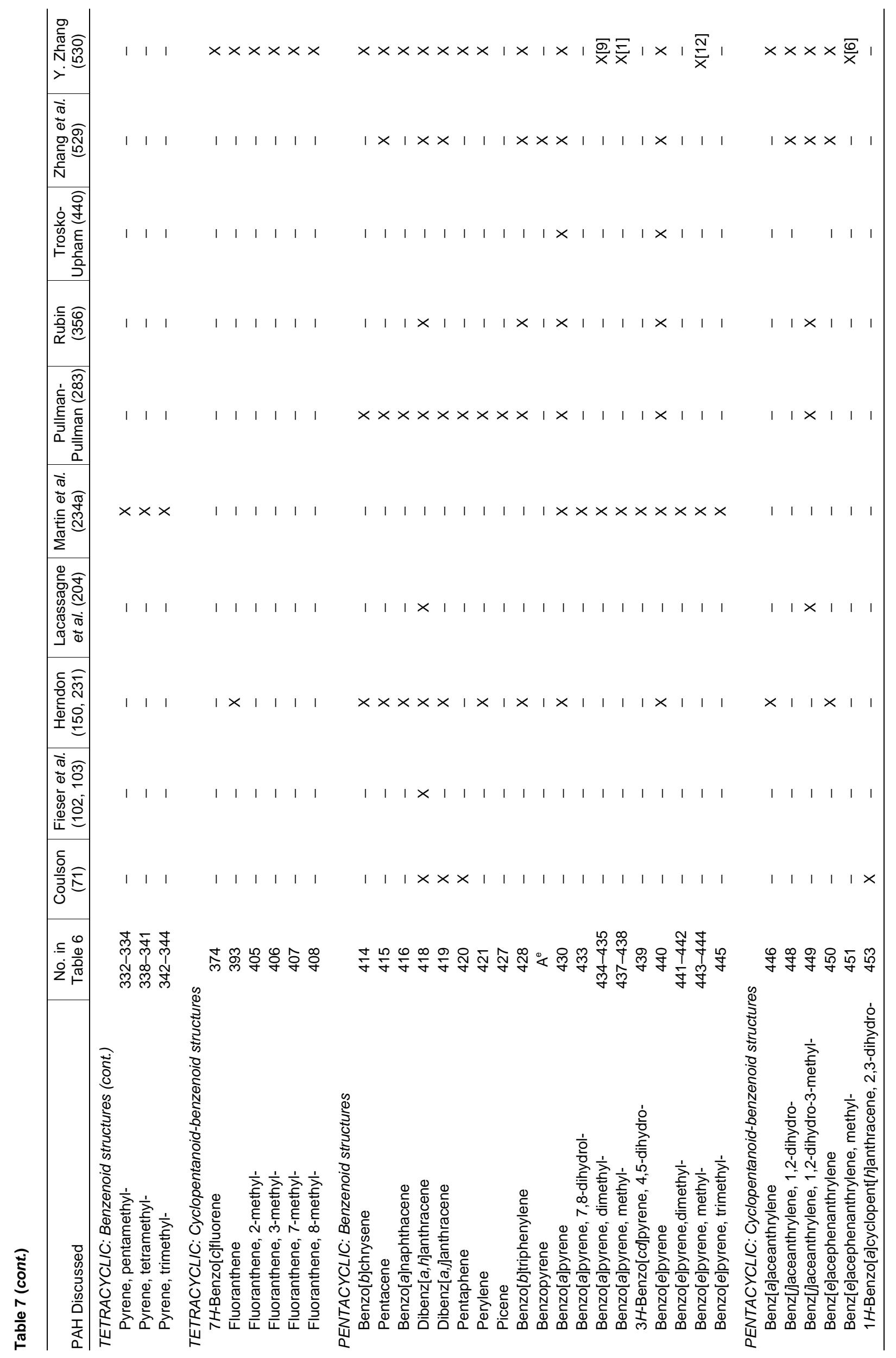




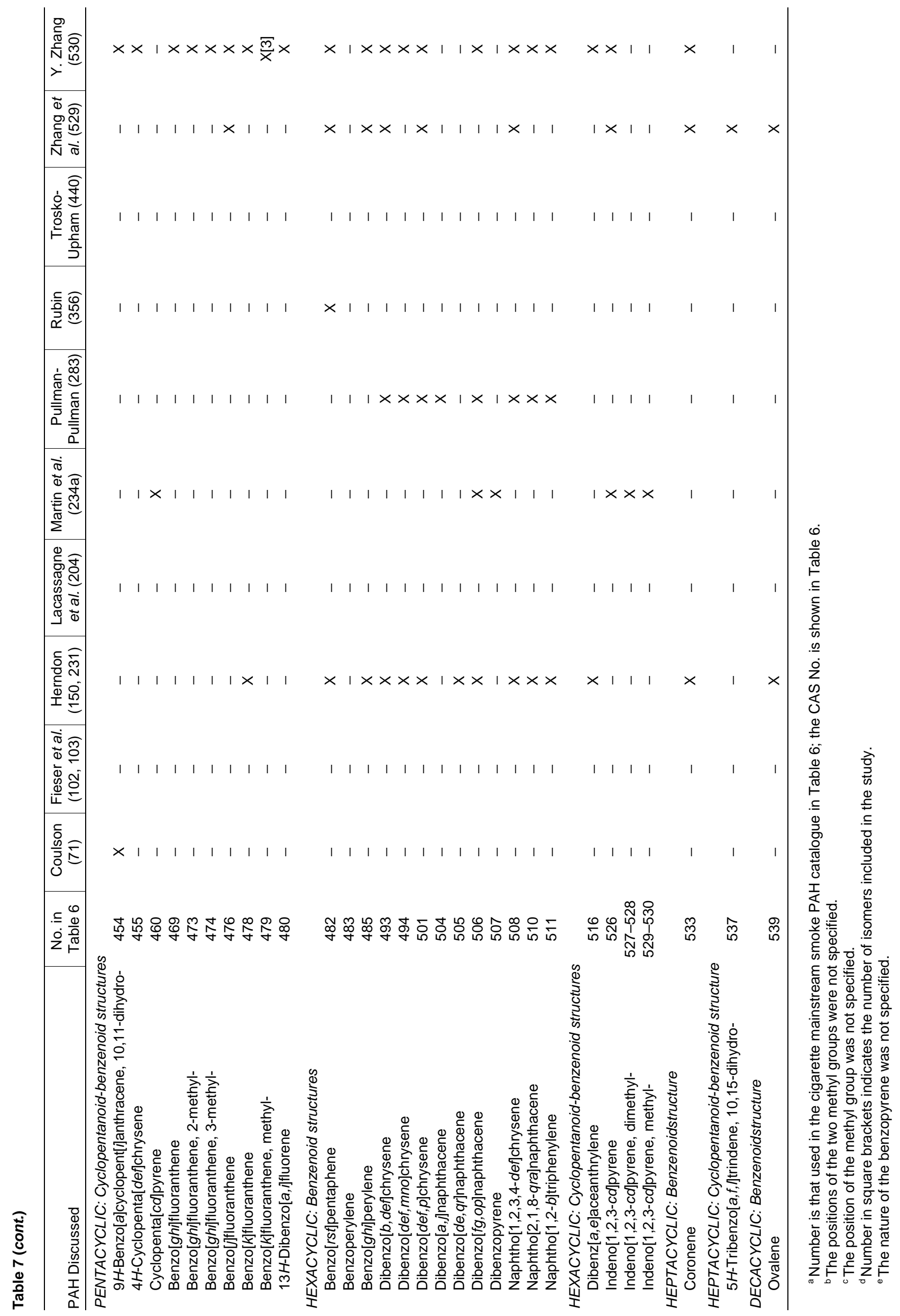


of PAHs, nearly 200 have been identified, many by the USDA group at Athens, GA (418). With the knowledge that CSC contains non-tumorigenic PAHs that have been shown to substantially reduce the tumorigenicity of several potently tumorigenic PAHs, consideration of the study of LACASSAGNE et al. raises several interesting questions with regard to tobacco smoke composition. 1) Do any of the benzacridines or other aza-arenes in CSC partially or totally inhibit the tumorigenicity of the tumorigenic benzacridines or other aza-arenes? 2) Do any of the benzacridines inhibit the tumorigenicity of tumorigenic PAHs? Do any of the PAHs reduce the tumorigenicity of the tumorigenic azaarenes?

The mixture known as CSC is so complex that it is not possible to ascribe its biological activity to any individual component because of the known behavior of that component when administered individually.

\section{REFERENCES}

1. Ahlmann, J.: Detection of polycyclic aromatic hydrocarbons in cigarette tar; Acta Pathol. Microbiol. Scand. 43 (1958) 379-390.

2. Akin, F.J., M.E. Snook, R.F. Severson, W.J. Chamberlain, and D.B. Walters: Identification of polynuclear aromatic hydrocarbons in cigarette smoke and their importance as tumorigens; J. Natl. Cancer Inst. 5 (1976) 191-195.

3. Alexandrov, K., P. Simova, and I. Savatinova: Potentielle kanzerogene Substanzen in Zigarettenrauch. Befund 3,4-Benzpyren [Potential carcinogenic substances in cigarette smoke. 3,4-Benzpyrene found]; Neoplasma 8 (1961) 575-576.

4. Allen, R.E.: A rapid method for the determination of polycyclic hydrocarbons in cigarette smoke; $30^{\text {th }}$ Tobacco Chemists' Research Conference, Program Booklet and Abstracts, Vol. 30, Paper No. 46, 1976, p. 32.

5. Allen, R.E. and D.G. Vickroy: The characterization of the smoke from Cytrel ${ }^{\circledR}$ smoking products and its comparison to smoke from flue-cured tobacco. III. Particulate phase analysis; Beitr. Tabakforsch. 8 (1976) 430-437.

6. Alvord, E.T. and S.Z. Cardon: Separation and identification of 3,4-benzpyrene in cigarette smoke; $9^{\text {th }}$ Tobacco Chemists' Research Conference, Program Booklet and Abstracts, Vol. 9, Part 2, Paper No. 24, 1955, p. 5; Inhibition of the formation of 3,4-benzpyrene from cigarette paper smoke; Ann. Mtg., Am. Assoc. Adv. Sci., Atlanta, GA (1955); The inhibition of the formation of 3,4-benzpyrene in cigarette smoke; Brit. J. Cancer 10 (1956) 498-503.

7. Appleton, R.A., C.R. Enzell, and B. Kimland: Tobacco chemistry. 3: Unsaturated hydrocarbon constituents of Greek tobacco; Beitr. Tabakforsch. 5 (1970) 266-274.

8. Arrendale, R.F., R.F. Severson, and M.E. Snook: Quantitative determination of naphthalenes in tobacco smoke by gas chromatography; Beitr. Tabakforsch. Int. 10 (1980) 100-105; Correction of error in Quantitative determination of naphthalenes in tobacco smoke by gas chromatography; [Beitr. Tabakforsch. Int. 10 (1980) 100-105]. Beitr. Tabakforsch. Int. 11 (1981) 55.
9. Ashburn, J.G.: Study of tobacco pretreatments; RDR, 1958, No. 20, December 10, see www.rjrtdocs.com $501008442-8528$.

10. R. J. Reynolds Tobacco Company (Ashburn, J.G. and A. Rodgman): Procedimento per il trattamento di tabacco [Procedure for the treatment of tobacco]; Italian Patent No. 593,317 (March 5, 1959); Ashburn, J.G. and A. Rodgman: Procédé de traitement du tabac [Procedure for the treatment of tobacco]; French Patent No. 1,206,210 (February 8, 1960).

11. Ayres, C.I. and R.E. Thornton: Determination of benzo $[a]$ pyrene and related compounds in cigarette smoke; Beitr. Tabakforsch. 3 (1965) 285-290.

12. Babin, J., D. Polic, and B. Neskovic: Detection of carcinogenic substances in wide use. I. The amount of 3,4-benzpyrene in the smoke of "Morava" cigarettes; Glasnik 14 (4) (1956) 45-52.

13. Badger, G.M., J.K. Donnelly, and T.M. Spotswood: The formation of aromatic hydrocarbons at high temperatures. XXIV. The pyrolysis of some tobacco constituents; Australian J. Chem. 18 (1965) 1249-1266.

14. Badger, G.M., S.D. Jolad, and T.M. Spotswood: The formation of aromatic hydrocarbons at high temperatures. XX. The pyrolysis of $\left[1-{ }^{14} \mathrm{C}\right]$ naphthalene; Australian J. Chem. 17 (1964) 771-777; The formation of aromatic hydrocarbons at high temperatures; Australian J. Chem. 19 (1966) 85; Australian J. Chem. 19 (1966) 95.

15. Baggett, M.S. and G.P. Morie: Selective removal of semivolatile components of cigarette smoke by various filters; $28^{\text {th }}$ Tobacco Chemists' Research Conference, Program Booklet and Abstracts, Vol. 28, Paper No. 7, 1974, p. 11; Beitr. Tabakforsch. 8 (1975) 150-152.

16. Bao, M., M. Sharifi, P. Joza, and T. Field: Determination of twenty polycyclic aromatic hydrocarbons in tobacco smoke by automated sample preparation and gas chromatography/mass spectrometry; $56^{\text {th }}$ Tobacco Science Research Conference, Program Booklet and Abstracts, Vol. 56, Paper No. 65, 2002, pp. 59-60.

17. Barkemeyer, H.: Eine neue Methode zur Bestimmung des 3,4-Benzpyrens in Tabakrauchkondensaten [A new method for the determination of 3,4-benzpyrene in tobacco smoke condensate]; Beitr. Tabakforsch. 1 (1962) 325-328.

18. Barry, G., J.W. Cook, G.A.D. Haslewood, C.L. Hewett, I. Hieger, and E.L. Kennaway: The production of cancer by pure hydrocarbons. Part III; Proc. Roy. Soc. (Biol.) 117 (1935) 318-351.

19. Beiträge zur Tabakforschung International: Seminar in tobacco science: Sidestream smoke; Beitr. Tabakforsch. Int. 17 (1997) 22-24.

20. Beiträge zur Tabakforschung International: Seminar in tobacco science: Tobacco smoke components; Beitr. Tabakforsch. Int. 17 (1997) 61-66.

20a.Bell, J.H.: Determination of benzo[a]pyrene in cigarette smoke condensate; Report, August 30, 1962, see www.Lorillarddocs.com 00118749 -8752.

21. Bell, J.H., M.S. Ireland, F.J. Schultz, and A.W. Spears: Identification of alkylated phenanthrenes in cigarette smoke condensate; $23^{\text {rd }}$ Tobacco Chemists' Research Conference, Program Booklet and Abstracts, Vol. 23, Paper No. 20, 1969, p. 12, see http://legacy.library.ucsf. edu/tid/ldf33c00, Bates No. 98427000. 
22. Bell, J.H., M.S. Ireland, F.J. Schultz, and A.W. Spears: Identification of certain polyaromatic hydrocarbons in cigarette smoke condensate; $24^{\text {th }}$ Tobacco Chemists' Research Conference, Program Booklet and Abstracts, Vol. 24, Paper No. 29, 1970, p. 21, see http://legacy. library.ucsf.edu/tid/jdf33c000, Bates No. 984269974.

23. Benner, J.F., H.R. Burton, and D. Burdick: Composition of cigarette smoke from high- and low-nitrate burley tobacco; Tob. Sci. 12 (1968) 37-40.

24. Benner, J.F., H.R. Burton, and D. Burdick: Temperature-yield profiles of tobacco and tobacco constituents. I. Borate-treated and untreated tobacco; Beitr. Tabakforsch. 5 (1969) 74-79.

24a. Benner, J.F., C.K. Keene, and T.W. Holt: Smoke analysis, condensate preparation and condensate fractionation; Proc. Univ. Kentucky Tob. Hlth. Workshop, 1973 Conference Report, Lexington, KY 4 (1973) 408-420.

25. Bentley, H.R.: A study of carcinogenic components in tobacco smoke; Brit. Emp. Cancer Camp., Ann. Rpt. 39 (1962) 25

26. Bentley, H.R. and E.G.N. Berry: The constituents of tobacco smoke: An annotated bibliography. Research Paper No. 3; Tobacco Manufacturers' Standing Committee, London (1959).

27. Bentley, H.R. and J.G. Burgan: Benzopyrene in tobacco and tobacco smoke; $12^{\text {th }}$ Tobacco Chemists' Research Conference, Program Booklet and Abstracts, Vol. 12, Paper No. 16, 1958, pp. 6-7; Polynuclear hydrocarbons in tobacco and tobacco smoke. I. 3,4-Benzpyrene; Analyst 83 (1958) 442-447.

28. Bentley, H.R. and J.G. Burgan: Polynuclear hydrocarbons in tobacco and tobacco smoke. II. The origin of 3,4-benzpyrene found in tobacco and tobacco smoke; Analyst 85 (1960) 723-727; III. The inhibition of the formation of 3,4-benzpyrene in cigarette smoke; Analyst 85 (1960) 727-730.

29. Bereman, R.D.: Method and product for removing carcinogens from tobacco smoke; U.S. Patent Application No. 20030000538 (January 2, 2003), Bereman, R.D.: Method of making a smoking composition; U.S. Patent No. 6789548 (September 14, 2004), see www.patentstorm.us/patents/6789548.html; Rainey, P. and R.D. Bereman: Method of making a smoking composition; U.S. Patent No. 6959712 (November 1, 2005), see www.patentstorm.us/patents/6959712.html.

30. Bilimoria, M.H., J. Johnson, M.A. Nisbet, S. Schmeller, and K.K. Georgieff: Inhibition of radical initiated polymerisation of vinyl acetate by tobacco smoke and some polycyclic hydrocarbons; Beitr. Tabakforsch. 7 (1973) 158-164.

31. Bonnet, J.: What carcinogenic substances have been demonstrated to be present in tobacco smoke condensate? Proc. $1^{\text {st }}$ Workshop Conf. on Lung Cancer Res. (App. A) (1958) 27-30.

32. Bonnet, J. and S. Neukomm: Sur la composition chimique de la fumée du tabac. I. Analyse de la fraction neutre [The chemical composition of tobacco smoke. I. Analysis of the neutral fraction]; Helv. Chim. Acta 39 (1956) 1724-1733.

33. Bonnet, J. and S. Neukomm: Résultats actuels des recherches chimiques sur la composition de la fumée du tabac [Current results on the chemical research on the composition of tobacco smoke]; Oncologia 10 (1957) 124-129.
34. Bonnet, J. and S. Neukomm: New investigations on carcinogenic substances in tobacco; Oncologia 12 (1959) 80-86.

35. Bonnet, J. and S. Neukomm: Carcinogenic and cocarcinogenic substances in tobacco smoke; Acta Unio Internat. Contra Cancrum 15 (1959) 561-563.

35a. Boyland, E., J.W. Gorrod, F.J.C. Roe, and B.V.C. Mitchley: The carcinogenicity of nitrosoanabasine, a possible constituent of tobacco smoke; Brit. Emp. Cancer Camp., Ann. Rpt. 41 (1964) 64; Boyland, E., F.J.C. Roe, and J.W. Gorrod: Induction of pulmonary tumours in mice by nitrosonornicotine, a possible constituent of tobacco smoke; Nature 202 (1964) 1126; Boyland, E., F.J.C. Roe, J.W. Gorrod, and B.V.C. Mitchley: The carcinogenicity of nitrosoanabasine, a possible constituent of tobacco smoke; Brit. J. Cancer 18 (1964) 265-272.

35b. Boyland, E. and F. Weigert: The metabolism of carcinogenic compounds; Brit. Med. Bull. 1947 (4) 354-359; Weigert, F. and J.C. Mottram: Intermediate stages in the metabolic conversion of benzopyrene to 8hydroxybenzopyrene in mice; Biochem. J. 37 (1943) 497-501; The biochemistry of benzopyrene. I. A survey, and new methods of analysis; Cancer Res. 6 (1946) 97-108; The biochemistry of benzopyrene. II. The course of its metabolism and the chemical nature of the metabolites; Cancer Res. 6 (1946) 109-120.

36. Bradford, J.A., W.R. Harlan, and H.R. Hanmer: Nature of cigaret smoke: Technic of experimental smoking; Ind. Eng. Chem. 28 (1936) 836-839.

37. British Empire Cancer Campaign: Aromatic polycyclic hydrocarbons in cigarette smoke; Brit. Emp. Cancer Camp., Ann. Rpt. 35 (1958) 303-305.

38. Brunnemann, K.D. and D. Hoffmann: Analysis of polynuclear aromatic hydrocarbons in the respiratory environment; Carc. Comp. Serv. 1 (1976) 283-297.

39. Brunnemann, K.D. and D. Hoffmann: [Chemical studies on tobacco smoke. LXXIV] Pyrolytic origins of major gas phase constituents of cigarette smoke; Recent Adv. Tob. Sci. 8 (1982) 103-140.

40. Burdick, D., J.F. Benner, and H.R. Burton: Thermal decomposition of tobacco. IV. Apparent correlations between thermogravimetric data and certain constituents in smoke from chemically-treated tobaccos; Tob. Sci. 13 (1969) 138-141.

41. Burgan, J.G.: The manufacturers' contribution to experimental research on smoking and lung cancer; Trans. Assoc. Ind. Med. Officers 9 (1959) 13-17.

42. Burton, H.R.: Thermal analyses of chemically-treated tobacco; Proc. University of Kentucky Tobacco Hlth. Workshop Conf. Rpt. 1 (1969) 33-38.

43. Campbell, J.M. and A.J. Lindsey: Polycyclic hydrocarbons extracted from tobacco. The effect upon total quantities found in smoking; Brit. J. Cancer 10 (1956) 649-652.

44. Campbell, J.M. and A.J. Lindsey: Polycyclic hydrocarbons in cigar smoke; Brit. J. Cancer 11 (1957) 192-195.

45. Candeli, A., A.J. Lindsey, and K. Persaud: Determination of polycyclic aromatic hydrocarbons in tobacco smoke; Anal. Chim. Acta 22 (1960) 458-461.

46. Candeli, A., A.J. Lindsey, and K. Persaud: Carta di sigarette al sulfammato di ammonio e idrocarburi cancerigeni [Cigarette paper containing ammonium sulfamate and carcinogenic hydrocarbons]; Bol. Soc. Ital. Biol. Sper. 36 (1960) 452-454. 
47. Cardon, S.Z.: 3,4-Benzpyrene in cigarette smoke; Tob. Sci. 2 (1958) 130-131.

48. Cardon, S.Z. and E.T. Alvord: The presence of 3,4benzpyrene in cigarette smoke; Ann. Mtg., Am. Assoc. Adv. Sci., Atlanta, GA (December 27, 1955), pp. 1-12.

49. Cardon, S.Z., E.T. Alvord, W.J. Rand, and R. Hitchcock: 3,4-Benzpyrene in the smoke of cigarette paper, tobacco, and cigarettes; Brit. J. Cancer 10 (1956) 485-497.

49a. Carpenter, R.D.: Benzo[ $[a]$ pyrene content of cigarette smoke-Comparison of PM Commander, Marlboro, and Winston; Memorandum to A. Bavley, May 30, 1964, see www.pmdocs.com 1001532767.

50. Carugno, N. and S. Rossi: Evaluation of polynuclear hydrocarbons in cigarette smoke by glass capillary columns; $19^{\text {th }}$ Tobacco Chemists' Research Conference, Program Booklet and Abstracts, Vol. 19, Paper No. 36 , 1965, pp. 49-51.

51. Chakraborty, B.B., K.D. Kilburn, and R.E. Thornton: Reduction in the concentration of aromatic polycyclic hydrocarbons in cigarette smoke; Chem. and Ind. (London) (1971) 672.

51a. Chakraborty, B.B. and R.E. Thornton: Aromatic polycyclic hydrocarbons; Report No. RD 609-R, October 9, 1966, see http://legacy.library.ucsf.edu/ tid/boo99e00, Bates No. 655041108/1184.

52. Chamberlain, W.J., R.L. Miller, and R.L. Stedman: Pilot studies on the composition of a polynuclear-enriched fraction of smoke condensate; $22^{\text {nd }}$ Tobacco Chemists' Research Conference, Program Booklet and Abstracts, Vol. 22, Paper No. 18, 1968, p. 11.

53. Chen, P.X. and S.C. Moldoveanu: Mainstream smoke chemical analyses for 2R4F Kentucky Reference Cigarette; Beitr. Tabakforsch. Int. 20 (2003) 448-458.

54. Chopra, N.M.: On pyrolysis, and the possible contribution of maleic hydrazide towards benzo(a)pyrene in tobacco smoke; Tob. Sci. 23 (1979) 29-30.

55. Chortyk, O.T. and W.S. Schlotzhauer: Studies on the pyrogenesis of tobacco smoke constituents (a review); Beitr. Tabakforsch. 7 (1973) 165-178.

56. Chortyk, O.T., W.S. Schlotzhauer, and R.L. Stedman: Composition studies on tobacco. XXIII. Pyrolytic and structural investigations on the polyphenol-amino acid pigments of leaf; Beitr. Tabakforsch. 3 (1966) 422-429.

57. Ciaravolo, S., G. Lionetti, M. Gionti, A. Nunziata, and E. Pierri: Microwave-assisted extraction: An efficient method for the determination of polycyclic hydrocarbons in particulate phase mainstream cigarette smoke; 2004 CORESTA Congress, Kyoto, Japan, Poster SS10.

58. Clar, E.: Zur Kenntnis mehrkerniger aromatischer Kohlenwasserstoffe und ihrer Abkömmlinge. I. Dibenzanthracene und ihrer Chinone [Knowledge of polynuclear aromatic hydrocarbons and their derivatives. I. Dibenzanthracene and its quinone]; Ber. Dtsch. Chem. Ges. 62 (1929) 350-359, 1378.

59. Clemo, G.R.: Some aspects of the chemistry of tobacco smoke. I; Tetrahedron 3 (1958) 168-174; Further aspects of the chemistry of tobacco smoke. II; Tetrahedron 11 (1960) 11-14.

60. Commins, B.T.: Formation of polycyclic aromatic hydrocarbons during pyrolysis and combustion of hydrocarbons; Atmos. Environ. 3 (1969) 565-572.

61. Commins, B.T., R.L. Cooper, and A.J. Lindsey: Polycyclic hydrocarbons in cigarette smoke; Brit. J. Cancer
8 (1954) 296-302.

62. Cook, J.W.: Chemical carcinogens and their significance; Lancet 1957 (i) 333-335.

63. Cook, J.W.: Chemistry of cancerogenic substances with special reference to lung cancer; Chem. Ind. (1957) $1282-1288$.

64. Cook, J.W., C.L. Hewett, and I. Hieger: Coal-tar constituents and cancer; Nature 130 (1932) 926; Isolation of a cancer-producing hydrocarbon from coal tar. II. Isolation of 1,2- and 4,5-benzopyrenes, perylene, and 1,2-benzanthracene; J. Chem. Soc. (1933) 395-398. Note: 1,2-Benzopyrene and 4,5-benzopyrene were the names originally assigned to 3,4-benzopyrene (benzo[a]pyrene) and 1,2-benzopyrene (benzo[e]pyrene), respectively.

65. Cook, J.W., R.A.W. Johnstone, and P.M. Quan: The composition of cigarette smoke. VIII. Some aromatic hydrocarbon constituents; Israel J. Chem. 1 (1963) 356-364.

66. Cooper, R.L., J.A.S. Gilbert, and A.J. Lindsey: Polycyclic hydrocarbons in cigarette smoke: The contribution made by the paper; Brit. J. Cancer 9 (1955) 442-445.

67. Cooper, R.L. and A.J. Lindsey: The presence of polynuclear hydrocarbons in cigarette smoke; Chem. and Ind. (London) (1953) 1205.

68. Cooper, R.L. and A.J. Lindsey: The presence of 3,4benzpyrene and other polycyclic hydrocarbons in the combustion products of cigarette paper; Chem. and Ind. (London) (1954) 1260-1261.

69. Cooper, R.L. and A.J. Lindsey: 3,4-Benzpyrene and other polycyclic hydrocarbons in cigarette smoke; Brit. J. Cancer 9 (1955) 304-309.

70. Cooper, R.L., A.J. Lindsey, and R.E. Waller: The presence of 3,4-benzpyrene in cigarette smoke; Chem. and Ind. (London) (1954) 1418.

70a. CORESTA Recommended Method No. 58: Determination of benzo $[a]$ pyrene in cigarette mainstream smoke - gas chromatography-mass spectrometry method; February, 2004.

71. Coulson, C.A.: Electronic configuration and carcinogenesis; Adv. Cancer Res. 1 (1953) 1-56.

72. Crabtree, H.G.: Anticarcinogenesis; Brit. Med. Bull. 4 (1947) 345-348.

72a. Cundiff, R.H and P.C. Markunas: Titrimetric analysis of 2,4,7-trinitrofluorenone complexes; Anal. Chem. 35 (1963) 1323-1324.

73. Cuzin, J.L.: What is evidence for the presence of significant amounts of carcinogens in cigarette paper? In cigarette tobacco? In cigarette additives? Proc. ${ }^{\text {st }}$ Conf. Lung Cancer Res. (1958) 49-50.

74. Cuzin, J.L.: Determination of anthracene, pyrene, and benzo[a]pyrene in condensates of tobacco smoke; SEITA, January, 1960.

75. Cuzin, J.L., M. Hubert-Habart, B. Muel, R. Roger, and R. Latarjet: La production du benzo-3,4-pyrene dans des cigarettes à papier imprégné de sulfamate d'ammonium [The production of 3,4-benzpyrene in cigarettes with paper impregnated with ammonium sulfamate]; Bull. Soc. Chim. France (1960) 982.

76. Cuzin, J.L., A. Testa, S. Testa, and G. Anguerra: Chemical and biological control of condensates from smoked tobacco treated after the Neukomm-Bonnet process; Z. Präventivmed. 28 (1963) 125-137.

77. Daudel, P. and R. Daudel: Chemical carcinogenesis and 
molecular biology; Interscience Publishers: London, 1966.

78. Davis, H.J., L.A. Lee, and T.R. Davidson: The fluorometric determination of benzo $[a]$ pyrene in cigarette smoke condensate; Anal. Chem. 38 (1966) 1752-1755.

79. Demole, E. and P. Enggist: A chemical study of Virginia tobacco flavour (Nicotiana tabacum L.). II. Isolation and synthesis of cis-2-isopropenyl-8-methyl1,2,3,4-tetrahydro-1-naphthalenol and 3-isopropenyl-5methyl-1,2-dihydronaphthalene; Helv. Chim. Acta 61 (1978) 1335-1341.

80. de Souza, T.L. and M. Scherback: The effect of glycerol added to tobacco on the constituents of cigarette smoke; Analyst 89 (1964) 735-739.

80a. Deutsch-Wenzel, R., H. Brune, G. Grimmer, and J. Misfeld: Local application to mouse skin as a carcinogen specific test system for nonvolatile $N$-nitroso compounds; Cancer Lett. 29 (1985) 85-92.

81. Dickey, J.B. and G.P. Touey: Tobacco research: Hydrocarbon components of the gaseous phase of cigarette smoke; Seminar presented at Research Dept., R.J. Reynolds Tobacco Company, Winston-Salem NC (March 1, 1956), edited by A. Rodgman, see www.rjrtdocs.com 503132566 -2594.

82. DiGiovanni, J., T.J. Slaga, D.L. Berry, and M.R. Juchau: Inhibitory effects of environmental chemicals on polycyclic aromatic hydrocarbon carcinogenesis; in: Carcinogenesis. A comprehensive survey. Vol. 5, edited by T.J. Slaga, Raven Press, New York, NY, 1980, pp. 145-168.

83. Dikun, P.P.: Use of the fine structure of the fluorescence spectrum of 3:4-benzpyrene to increase the certainty of its detection; Voprosy Onkol. 5 (12) (1959) 672-677.

84. Dikun, P.P. and S.G. Chushkin: Fluorescence spectral analysis of the products of tobacco smoke; Voprosy Onkol. 5(7) (1959) 34-37.

85. Dikun, P.P., S.G. Chushkin, A.L. Gritsiute, and A.L. Mironova: The results of studies on the possible carcinogenic effect of tobacco products; Voprosy Onkol. 6 (1960) 603-604.

86. Dikun, P.P., I.A. Kalinina, N.D. Krasnitskaya, and V.A. Tokovoi: Absorption of 3,4-benzpyrene from tobacco smoke by various filtering materials; Voprosy Onkol. 11 (1965) 86-89.

87. Dikun, P.P., N.D. Krasnitskaya, and S.G. Chushkin: Some data on the content of 3,4-benzpyrene in tobacco smoke; Voprosy Onkol. 8 (1962) 31-35.

88. Dipple, A., R.C. Moschel, and C.A.H. Bigger: Polynuclear hydrocarbons; Chapter 2, in: Chemical carcinogens. Second edition, edited by C.E. Searle, American Chemical Society Monograph 182, American Chemical Society, Washington, DC, 1984, pp. 41-163.

88a. Dobrovolskaia-Zavadskaia, N.: Doses of 1,2,5,6-dibenzanthracene capable of producing cancer in mice; Compt. Rend. Soc. Biol. 129 (1938) 1055-1057.

89. Doll, R.: Smoking and lung cancer: Report to the subcommittee for the study of the risks of cancer from air pollution and the consumption of tobacco; Acta Unio Internat. Contra Cancrum 15 (1959) 1283-1296.

90. Druckrey, H.: Experimental investigations on the possible carcinogenic effects of tobacco smoking; Acta Med. Scand. Suppl. 369 (1961) 24-42.

90a. Druckrey, H. and R. Preussmann: Zur Entstehung carcinogener Nitrosamine am Beispiel des Tabakrauchs
[About the formation of carcinogenic nitrosamines in tobacco smoke]; Naturwissenschaft. 49 (1962) 498-499.

91. Druckrey, H. and A. Schildbach: Quantitative Untersuchungen zur Bedeutung des Benzpyrens für die carcinogene Wirkung von Tabakrauch [Quantitative investigation of the significance of benzpyrene in the carcinogenic action of tobacco smoke]; Z. Krebsforsch. 65 (1963) 465-470.

92. Druckrey, H., D. Schmähl, H. Beuthner, and F. Muth: Comparative investigation of the carcinogenic action in rats of tobacco condensate, tobacco extract, and benzpyrene; Naturwissenschaften 47 (1960) 605-606.

93. Eatough, D.J., L.D. Hansen, and E.A. Lewis: The chemical characterization of environmental tobacco smoke; in: Environmental tobacco smoke, edited by D.J. Ecobichon and J.M. Wu, Proc. Internat. Symp. at McGill University, Montreal, PQ, 1989, Lexington Books, D.C. Heath and Company, Lexington, MA (1990) 3-39; Environ. Tech. 11 (1990) 1071-1085.

94. Edmunds, F.S. and R.A.W. Johnstone: Constituents of cigarette smoke. IX. The pyrolysis of polyenes and the formation of aromatic hydrocarbons; J. Chem. Soc. (1965) 2892-2897, 2898-2900.

95. Elmenhorst, H.: Determination of naphthalenes in Latakia tobacco; $7^{\text {th }}$ Internat. Tob. Sci. Cong., Manila, The Philippines, 1980, CORESTA Inf. Bull., Spec. Edition 1980: Paper S05, 120-121.

96. Elmenhorst, H. and G. Grimmer: Polycyclische Kohlenwasserstoffe aus Zigarettenrauchkondensat. Eine Methode zur Fraktionierung grosser Mengen für Tierversuche [Polycyclic hydrocarbons from cigarette smoke condensate. A method for fractionating large quantities]; Z. Krebsforsch. 71 (1968) 66-73.

97. Elmenhorst, H. and G. Reckzeh: Aromatische Kohlenwasserstoffe im Tabakrauch [Aromatic hydrocarbons in tobacco smoke]; Beitr. Tabakforsch. 2 (1964) 180-204.

98. Elmenhorst, H. and G. Schultz: Flüchtige Inhaltsstoffe des Tabakrauches. Die chemischen Bestandteile der Gas-Dampf-Phase [Volatile components of tobacco smoke. The chemical constituents of the vapor phase]; Beitr. Tabakforsch. 4 (1968) 90-123.

99. Entwhistle, I.D. and R.A.W. Johnstone: Constituents of cigarette smoke. IX. The isolation and synthesis of acenaphthylenes and monocyclic polyolefins; J. Chem. Soc. (1968) 1818-1822.

100. Falk, H.L. and P Kotin: The experimental production of hydrocarbons in simulated cigarette smoking: Their analysis and quantitation; Progress Report No. 1, December 14, 1955, see http://legacy.library.ucsf.edu/ tid/xxt6aa00; Falk, H.L.: The experimental production of hydrocarbons in simulated cigarette smoking: Their analysis and quantitation; Progress Report No. 2, February 1-November 20, 1956, see http://legacy. library.ucsf.edu/tid/uxt6aa00.

101. Falk, H.L., P. Kotin, and S. Thompson: Inhibition of carcinogenesis. The effect of hydrocarbons and related compounds; Arch. Environ. Hlth. 9 (1964) 169-179.

102. Fieser, L.F.: [Oxidation-reduction potential of quinones]; J. Am. Chem. Soc. 50 (1928) 465; J. Am. Chem. Soc. 51 (1929) 3101; J. Am. Chem. Soc. 52 (1930) 4915; J. Am. Chem. Soc. 53 (1930) 5204; Fieser, L.F. and E.M. Dietz: [Oxidation-reduction potential of quinones]; J. Am. Chem. Soc. 53 (1931) 1128; Fieser, L.F. and M. Fieser: [Oxidation-reduction potential of quinones]; J. Am. Chem. Soc. 53 (1934) 1565; Fieser, L.F. 
and M.A. Peters: [Oxidation-reduction potential of quinones]; J. Am. Chem. Soc. 53 (1931) 793; J. Am. Chem. Soc. 53 (1931) 4080.

103. Fieser, L.F.: Theory of the structure and reactions of aromatic compounds; Chapter 3 , in: Organic chemistry: An advanced treatise, edited by H. Gilman, John Wiley and Sons, Inc., New York, NY, 1942, pp. 117-213; see 158-174.

104. Fieser, L.F.: Chemical carcinogenesis; Arthur Stolle Festschrift (1957) 489-498.

105. Fieser, L.F.: More on smoking and health; Chemistry 1964 (3) 18-19.

105a.Fieser, L.F. and E.M. Dietz: [Synthesis of benz $[a]$ anthracene and dibenz $[a, h]$ anthracene $]$; Ber. Dtsch. Chem. Ges. 62 (1929) 1827-1833.

106. Fowles, J. and M. Bates: The chemical constituents in cigarettes and cigarette smoke: Priorities for harm reduction; A Report to the New Zealand Ministry of Health, March 2000, pp. 1-65.

107. Fredrickson, J.D.: Process for increasing the volume of tobacco; RDR, 1965, No. 3, January 18, see www.rjrtdocs.com 500965533 -5569; Process for increasing the filling volume of tobacco. Addendum to RDR, 1965, No. 3; RDM, 1965, No. 58, August 5, see www.rjrtdocs.com 502476759 -6762.

108. Frost, B.E., G. McKenna, and M.S. Williams: The measurement of benzo $[a]$ pyrene in mainstream cigarette smoke; 52 ${ }^{\text {nd }}$ Tobacco Science Research Conference, Program Booklet and Abstracts, Vol. 52, Paper No. 31, 1998, p. 29.

109. Fujimora, T., R. Kasuga, H. Matsushita, and M. Noguchi: Neutral aroma constituents in burley tobacco; Agr. Biol. Chem. Japan 40 (1976) 303-315.

110. Galuskinova, V.: 3,4-Benzpyrene determination in the smoky atmosphere of social meeting rooms and restaurants. A contribution to the problem of the noxiousness of so-called passive smoking; Neoplasma 11 (1964) 465-469.

111. George, T.W. and C.H. Keith: The selective filtration of tobacco smoke; Chapter XI, in: E.L. Wynder and D. Hoffmann: Tobacco and tobacco smoke: Studies in experimental carcinogenesis, Academic Press, New York, NY, 1967, pp. 577-622.

112. Gil-Av, E. and J. Shabtai: Precursors of carcinogenic hydrocarbons in tobacco smoke; Nature 197 (1963) 1065-1066.

113. Gilbert, J.A.S. and A.J. Lindsey: Polycyclic hydrocarbons in cigarette smoke: The amounts held in stubs and ash; Brit. J. Cancer 10 (1956) 642-645.

114. Gilbert, J.A.S. and A.J. Lindsey: Polycyclic hydrocarbons in tobacco smoke: Pipe smoking experiments; Brit. J. Cancer 10 (1956) 646-648.

115. Gilbert, J.A.S. and A.J. Lindsey: The thermal decomposition of some tobacco constituents; Brit. J. Cancer 11 (1957) 398-402.

116. Gori, G.B. (Editor): Report No. 1. Toward less hazardous cigarettes. The first set of experimental cigarettes; DHEW Publ. No. (NIH) 76-905 (1976).

117. Gori, G.B. (Editor): Report No. 2. Toward less hazardous cigarettes. The second set of experimental cigarettes; DHEW Publ. No. (NIH) 76-1111 (1976).

118. Gori, G.B. (Editor): Report No. 3. Toward less hazardous cigarettes. The third set of experimental cigarettes; DHEW Publ. No. (NIH) 77-1280 (1977).

119. Gori, G.B. (Editor): Report No. 4. Toward less hazardous cigarettes. The fourth set of experimental cigarettes; DHEW Publ. (NIH) (March, 1980).

120. Graham, J.F.: A fraction trapping and transfer device for the analysis of cigarette smoke; Beitr. Tabakforsch. 5 (1969) 43-51.

121. Graham, J.F.: Cigarette smoke analysis by computerGLC; Beitr. Tabakforsch. 5 (1970) 220-228.

122. Grasso, P.: Carcinogens in food; Chapter 19, in: Chemical carcinogens, Second edition, edited by C.E. Searle, American Chemical Society Monograph 182, American Chemical Society, Washington, DC, 1984, pp. 1205-1239.

123. Green, C.R. and F.W. Best: Smoke composition: Comparison of J-10 and J-10T smokes with a tobacco control; RDR, 1974, No. 6, August 26, see www.rjrtdocs.com $510691758-1783$.

124. Green, C.R., F.W. Best, M.P. M.P. Newell, R.A. Lloyd, and C.W. Miller: Semi-quantitative comparison of smoke from J-10:tobacco and tobacco cigarettes; RDR, 1976, No. 4, February 18, see www.rjrtdocs.com 501004048 -4075; 510628992 -9019.

125. Green, C.R. and A. Rodgman: The Tobacco Chemists' Research Conference: A half century forum for advances in analytical methodology of tobacco and its products; Recent Adv. Tob. Sci. 22 (1996) 131-304.

126. Green, C.R. and J.N. Schumacher: Smoke composition: puffed vs. unpuffed tobacco; RDR, 1971, No. 7. February 26, see www.rjrtdocs.com 501001966-1998.

127. Green, C.R., L. Vestal, and J.N. Schumacher: The investigation of the cigarette smoke from Celanese smoking material; RDR, 1969, No. 32, September 19, see www.rjrtdocs.com 500969764 -9795.

128. Green, C.R., L.L. Vestal, and J.N. Schumacher: The investigation of the cigarette smoke from the Sutton smoking material; see www.rjirtdocs.com 501001439 1463; see also Part V (see www.rjrtdocs.com 517550453 -0477), in: Rodgman, A, J.D. Fredrickson, E.S. Hickman, M.P. Newell, J.N. Schumacher, C.R. Green, F.W. Best, G.W. Spence, R.E. Shackelford, E.D. Harper Jr, and L.L. Vestal: The Sutton Research Corporation smoking material; RDR, 1970, No. 48, December 4, see www.rjrtdocs.com 501001173 -1175, 501001176 -1189, 501001190 -1289, 501001290 $1406,501001407-1438,501001439-1463,501001464$ $-1468, \quad 501001469-1475, \quad 501001526-1539$, $501001540-1591,501001592-1772,501001773$ 1783, $501001784-1788$.

129. Gregg, E., C. Hill, M. Hollywood, M. Kearney, K. McAdam, D. McLaughlin, S. Purkis, and M. Williams: The UK smoke constituents testing study. Summary of results and comparison with other studies; Beitr. Tabakforsch. Int. 21 (2004) 117-138.

130. Grimmer, G.: Eine Methode zur Bestimmung von 3,4Benzpyren in Tabakrauchkondensaten [A method for the estimation of 3,4-benzpyrene in tobacco smoke condensate]; Beitr. Tabakforsch. 1 (1961) 107-116.

131. Grimmer, G.: Polycyclische aromatische Kohlenwasserstoffe - ihre Vorkommen und ihre Bestimmung [Polycyclic aromatic hydrocarbons - their occurrence and estimation]; Lebensmittelchem. Gericht. Chem. 35 (1981) 67-71.

131a. Grimmer, G., H. Brune, G. Dettbarn, J. Jacob, J. Misfeld, U. Mohr, K.W. Naujack, J. Timm and R. Wenzel-Hartung: Contribution of polycyclic aromatic hydrocarbons and other polycyclic aromatic com- 
pounds to the carcinogenicity of combustion source and air pollution; Environ. Sci. Res. 39 (1990) 127-140; Relevance of polycyclic aromatic hydrocarbons as environmental carcinogens; Fresenius J. Anal. Chem. 339 (1991) 792-795.

132. Grimmer, G., H. Brune, R. Deutsch-Wenzel, G. Dettbarn,. and J. Misfeld: Contribution of polycyclic aromatic hydrocarbons to the carcinogenic impact of gasoline engine exhaust condensate evaluated by implantation into the lungs of rats; J. Natl. Cancer Inst. 72 (1984) 733-739; Grimmer, G., H. Brune, R. Deutsch-Wenzel, K.W. Naujack, J. Misfeld, and J. Timm: On the contribution of polycyclic aromatic hydrocarbons to the carcinogenic impact of automobile exhaust condensate evaluated by local application onto mouse; Cancer Lett. 21 (1983) 105-113; Grimmer, G., H. Brune, R. Deutsch-Wenzel, G. Dettbarn, J. Jacob, K.W. Naujack, U. Mohr, and H. Ernst: Contribution of polycyclic aromatic hydrocarbons and nitro-derivatives to the carcinogenic impact of diesel engine exhaust condensate evaluated by implantation into the lungs of rats; Cancer Lett. 37 (1987) 173-180.

133. Grimmer, G., A. Glaser, and G. Wilhelm: Die Bildung von Benzo[ $a]$ pyren und Benzo[ $e]$ pyrene beim Erhitzen von Tabak in Abhängigkeit von Temperatur und Strömungsgeschwindigkeit in Luft- und Stickstoffatmosphäre [The formation of benzo $[a]$ pyrene and benzo[ $e]$ pyrene during the heating of tobacco in correlation to temperature and flow rate in air and nitrogen atmospheres]; Beitr. Tabakforsch. 3 (1966) 415-421.

133a. Grimmer, G. and K.W. Naujack: Gas chromatographic determination of polycyclic aromatic hydrocarbons in sidestream smoke and indoor air; in: Environmental carcinogens: Methods of analysis and exposure measurement. passive smoking, edited by I.K. O'Neill, K.D. Brunnemann, B. Dodet, and D. Hoffmann, IARC, Lyon, France, IARC Sci. Publ. No. 81 (1987) 249-268; Grimmer, G., K.W. Naujack, and G. Dettbarn: Gas chromatographic determination of polycyclic aromatic hydrocarbons, aza-arenes, aromatic amines in the particle and vapor phase of mainstream and sidestream smoke of cigarettes; Internat. Exptl. Toxicol. Symp. on Passive Smoking, Essen FRG (1986) pp. 1-19; Toxicol. Lett. 35 (1987) 117-124; Grimmer, G., H. Brune, G. Dettbarn, K.W. Naujack, U. Mohr, and R. Wenzel-Hartung: Contribution of polycyclic aromatic hydrocarbons to the carcinogenicity of sidestream smoke of cigarettes evaluated by implantation into the lung of rats; Cancer Lett. 43 (1988) 173-177.

134. Grob, K. and J.A. Völlmin: GC-MS analysis of the "semi-volatiles" of cigarette smoke; J. Chromat. Sci. 8 (1970) 218-220.

135. Grob, K. and J.A. Völlmin: Analyse der "SemiVolatiles" aus Cigarettenrauch mit Hilfe einer Kombination von hochauflösender Gaschromatographie und Massenspektrometrie [The determination of "semivolatiles" in cigarette smoke by the combination of gas chromatography with mass spectrometry]; Beitr. Tabakforsch. 5 (1969) 52-57.

135a. Guan, J.: Investigation of carbon monoxide and benzo $[a]$ pyrene abatement in cigarette smoke through the application of lanthanum manganese oxide and manganese oxides; $58^{\text {th }}$ Tobacco Chemists' Research Conference, Program Booklet and Abstracts, Vol. 58, Paper No. 71, 2004, p. 66.
136. Guérin, M.: Étude sur la pouvoir cocarcinogène du goudron de fumée de cigarette [Study of the cocarcinogenic activity of cigarette smoke tar]; Bull. Assoc. Franc. Étude Cancer 48 (1961) 365-376.

137. Guerin, M.R., R.A. Jenkins, and B.A. Tomkins: The chemistry of environmental tobacco smoke: Composition and measurement; Lewis Publishers, Ann Arbor MI/Boca Raton FL, 1992, see pp. 230-235.

138. Guvernator, G.C. III, P.L. Gager Jr, E.W. Robb, and A. Bavley: Electron capture detection of gas-chromatographed polycyclic hydrocarbons; J. Gas Chromatog. 3 (1965) 363-367.

139. Haeberer, A.F. and O.T. Chortyk: High pressure liquid chromatography of polynuclear aromatic hydrocarbon constituents; Recent Adv. Tob. Sci. 1 (1975) 72-96.

140. Haeberer, A.F. and O.T. Chortyk: Analysis of volatile pyrolytic products of tobacco constituents: Stearic acid pyrolysis; Beitr. Tabakforsch. 8 (1975) 141-144.

141. Haeberer, A.F., M.E. Snook, and O.T. Chortyk: Reverse-phase high-pressure liquid chromatography of polynuclear aromatic hydrocarbons; $28^{\text {th }}$ Tobacco Chemists' Research Conference, Program Booklet and Abstracts, Vol. 28, Paper No. 41, 1974, p. 28; Liquid chromatography of polynuclear aromatic hydrocarbons of cigarette smoke condensate; Anal. Chim. Acta 80 (1975) 303-309.

142. Harke, H.P., D. Schüller, J. Klimisch, and K. Meissner, Investigation of polycyclic aromatic hydrocarbons in cigarette smoke; Z. Lebensm. Unters. Forsch. 162 (1976) 291-297.

143. Harris, J.L.: A treatise on theories of polycyclic aromatic hydrocarbon formation; RDM, 1967, No. 38, July 26, see www.rjrtdocs.com $504913304-3311$; Control of polycyclic aromatic hydrocarbons in cigarette smoke. A survey; RDM, 1967, No. 46, August 21, see www.rjrtdocs.com $500613447-3454$.

144. Hartwell, J.L.: Survey of compounds which have been tested for carcinogenic activity; USPHS Publ. No. 149 (1947) Washington, DC; Survey of compounds which have been tested for carcinogenic activity; USPHS Publ. No. 149, 2nd Edition (1951), Washington, DC.

145. Hecht, S.S.: Tobacco smoke carcinogens and lung cancer; J. Natl. Cancer Inst. 91 (1999) 1194-1210.

146. Hecht, S.S., W.E. Bondinell, and D. Hoffmann: Isolation and identification of alkylchrysenes in cigarette smoke; $27^{\text {th }}$ Tobacco Chemists' Research Conference, Program Booklet and Abstracts, Vol. 27, Paper No. 32, 1973, p. 23; Chrysene and methylchrysenes: Presence in tobacco smoke and carcinogenicity; J. Natl. Cancer Inst. 53 (1974) 1121-1133; Hecht, S.S., M. Loy, R. Maronpot, and D. Hoffmann A study of chemical carcinogenesis: Comparative carcinogenicity of 5-methylchrysene, benzo[a]pyrene, and modified chrysenes; Cancer Lett. 1 (1976) 147-154.

146a. Hecht, S.S. and D. Hoffmann: 4-(N-Methylnitrosamino)-1-(3-pyridyl)-1-butanone, a nicotine-derived tobacco-specific nitrosamine, and cancer of the lung and pancreas in humans; in: The origins of human cancer: A comprehensive review, edited by J. Brugge, T. Curran, E. Harlow, and F. Cormick, Cold Spring Harbor Laboratory, Cold Spring Harbor, NY, 1991, 745-755.

147. Heckman, R.A. and F.W. Best: An investigation of the lipophilic bases of cigarette smoke condensate; Tob. Sci. 25 (1981) 33-39. 
148. Hege, R.B. Jr: Determination of benzo $[a]$ pyrene in smoke condensate by ultraviolet spectroscopy; RDM, 1971, No. 58, December 7, see www.rjrtdocs.com $500605845-5851$.

149. Hege, R.B. Jr: Modification of routine thin-layer method for determining benzo[ $a]$ pyrene in smoke; RDM, 1972, No. 13, March 8, see www.rjrtdocs.com $500615425-5432$.

150. Herndon, W.C.: Theory of carcinogenic activity of aromatic hydrocarbons; Trans. N.Y. Acad. Sci. Ser. II 36 (1974) 200-217; Herndon, W.C. and A.J. Bruce; Perimeter codes for benzenoid aromatic hydrocarbons; in: Studies in physical and theoretical chemistry, edited by R.B. King and D.H. Rouvray, Elsevier Science Publishers B.V., Amsterdam 51, 1987, pp. 491-513; Linear notation for benzenoid aromatic hydrocarbons. Molecular similarity based on notation similarity; J. Math. Chem. 2 (1988) 155-169; Herndon, W.C., P.C. Nowak, D.A. Connor, and P. Lin; Empirical model calculations for thermodynamic and structural properties of condensed polycyclic aromatic hydrocarbons; J. Am. Chem. Soc. 114 (1992) 41-47; Herndon, W.C. and L.V. Szentpaly: Theoretical model of activation of carcinogenic polycyclic benzenoid aromatic hydrocarbons. Possible new classes of carcinogenic aromatic hydrocarbons; J. Molecul. Structure (Theochem) 148 (1986) 141-152; Structure-property relationships in polycyclic aromatics; Symposium on Chem. Polynuclear Aromatic Hydrocarbons, Am. Chem. Soc. (1986) 849-855.

151. Herrmann, K.: Über die phenolischen Inhaltstoffe des Tabaks und des Tabakrauches [Phenols in tobacco leaves and tobacco smoke]; Beitr. Tabakforsch. 2 (1964) 159-179.

152. Hoffmann, D. and S.S. Hecht: Advances in tobacco carcinogenesis; Chapter 3, in: Chemical carcinogenesis and mutagenesis, edited by C.S. Cooper and P. Grover, I, Springer-Verlag, London, UK, 1990, pp. 63-102.

153. Hoffmann, D. and I. Hoffmann: [Chemical studies on tobacco smoke. C] The changing cigarette: 1950-1995; J. Toxicol. Environ. Hlth. 50 (1997) 307-364.

154. Hoffmann, D. and I. Hoffmann: Tobacco smoke components. Letter to the Editor; Beitr. Tabakforsch. Int. 18 (1998) 49-52.

155. Hoffmann, D. and I. Hoffmann: [Cigar smoke:] Chemistry and toxicology; Smoking and Tobacco Control Monograph 9 (1998) 55-104.

156. Hoffmann, D. and I. Hoffmann: The changing cigarette: Chemical studies and bioassays; Chapter 5, in: Risks associated with smoking cigarettes with low machinemeasured yields of tar and nicotine, NCI Smoking and tobacco control, Monograph 13, edited by D.M. Burns and N.L. Benowitz, Bethesda, MD, 2001, pp. 159-191.

157. Hoffmann, D., I. Hoffmann, and K. El-Bayoumy: The less harmful cigarette: A controversial issue. A tribute to Ernst L. Wynder; Chem. Res. Toxicol. 14 (2001) 767-790

157a. Hoffmann, D. and G. Rathkamp: [Chemical studies on tobacco smoke. XIV.] Quantitative determination of fluorenes in cigarette smoke and their formation by pyrosynthesis; Anal. Chem. 44 (1972) 899-904.

157b.Hoffmann, D., G. Rathkamp, S. Nesnov, and E.L. Wynder: Chemical studies on tobacco smoke. XVI. Fluoranthenes: Quantitative determination in cigarette smoke, formation by pyrolysis, and tumor initiation activity; J. Natl. Cancer Inst. 49 (1972) 1165-1175.

158. Hoffmann, D., G. Rathkamp, and E.L. Wynder: Comparison of the yields of several selected components in the smoke from different tobacco products; J. Natl. Cancer Inst. 31 (1963) 627-637.

159. Hoffmann, D., G. Rathkamp, and E.L. Wynder: Chemical studies on tobacco smoke. IX. Quantitative analysis of chlorinated hydrocarbon insecticides; Beitr. Tabakforsch. 5 (1969) 140-148.

160. Hoffmann, D., A. Rivenson, F.L. Chung, and E.L. Wynder: Potential inhibitors of tobacco carcinogenesis; in: Tobacco smoking and nutrition: Influence of nutrition on tobacco-associated health risks, edited by J.N. Diana and W.A. Pryor, Ann. N.Y. Acad. Sci. 686 (1993) 140-160.

160a. Hoffmann, D., I. Schmeltz, S.S. Hecht, and E.L. Wynder: Tobacco carcinogenesis; in: Polycyclic hydrocarbons and cancer, Vol. 1, Chemistry, molecular biology and environment, edited by H.V.. Gelboin and P.O. Ts'o, Academic Press, New York, NY, 1978, pp. 85-117.

161. Hoffmann, D. and E.L. Wynder: Identification of polynuclear aromatic hydrocarbons; $136^{\text {th }}$ Natl. Mtg., Am. Chem. Soc., Atlantic City, NJ (1959) Paper No. 16U.

162. Hoffmann, D. and E.L. Wynder: Short-term determination of carcinogenic aromatic hydrocarbons; Anal. Chem. 32 (1960) 295-296.

163. Hoffmann, D. and E.L. Wynder: On the isolation and identification of polycyclic aromatic hydrocarbons; Cancer 13 (1960) 1062-1073.

164. Hoffmann, D. and E.L. Wynder: The reduction of tumorigenicity of cigarette smoke condensate by addition of sodium nitrate to tobacco; Cancer Res. 27 (1967) 172-174.

165. Hoffmann, D. and E.L. Wynder: Selective reduction of the tumorigenicity of tobacco smoke. Experimental approaches; in: Toward a less harmful cigarette, edited by E.L. Wynder and D. Hoffmann, Natl. Cancer Inst. Monograph 28 (1968) 151-172.

166. Hoffmann, D. and E.L. Wynder: A study of tobacco carcinogenesis. XI. Tumor initiators, tumor accelerators, and tumor promoting activity of condensate fractions; Cancer 27 (1971) 848-864.

167. Hoffmann, D. and E.L. Wynder: Chemical constituents and bioactivity of tobacco smoke; in: Tobacco: A major health hazard, edited by D.G. Zardidze and R. Peto, IARC, Lyon, France, IARC Sci. Publ. No. 74 (1986) $145-165$.

168. Holzer, G., J. Oró, and W. Bertsch: Gas chromatographic-mass spectrometric evaluation of exhaled tobacco smoke; J. Chromatog. 126 (1976) 771-785.

169. Hubert-Habart, M.R.: Le benzo-3,4 pyrène pyroformé dans les produits de combustion de la cigarette: Détection, dosage, recherche des facteurs d'inhibition [Pyrogenesis of 3,4-benzpyrene in the products from cigarette combustion: Detection, yield, research on inhibition factors]; Thesis, University of Paris (1960).

170. Hubert-Habart, M., R. Latarjet, D. Lavalette, B. Muel, L. René, and R. Royer: Comparaison des quantités de benzo-3,4 pyrène formées par combustion de divers types de papiers à cigarettes [Comparison of the quantities of 3,4-benzpyrene formed by the combustion of different types of cigarette papers]; Bull. Cancer 53 (1958) 53-56.

171. Hubert-Habart, M., D. Lavalette, and R. Latarjet: Sur la 
formation du benzo-3,4 pyrène lors de la combustion de papier à cigarettes préalablement irradié [Formation of 3,4-benzpyrene during the combustion of previously irradiated cigarette paper]; Bull. Soc. Chim. Franc. (1964) 3033.

172. Hubert-Habart, M., B. Muel, R. Royer, and R. Latarjet: Effet inhibiteur de sels mineraux oxygénés sur la formation du benzo-3,4 pyrène lors de combustion du papier à cigarettes [Effect of oxygenated mineral salts on the formation of 3,4-benzpyrene during the combustion of cigarette papers]; Compt. Rend. Acad. Sci. 246 (1958) 1440-1441.

173. Ikeda, S.: Contribution to the study of tobacco smoke; Sci. Papers, Inst. Phys. Chem. Res. (Tokyo) 42 (1947) 80.

174. International Agency for Research on Cancer (IARC): Evaluation of the carcinogenic risk of chemicals to humans: Tobacco smoking; IARC, Lyon, France, IARC Monograph 38 (1986) 83-126, 387-394.

175. Ishiguro, S. and S. Sugawara: Comparisons of smoke components in the semivolatile phase from lamina and midrib cigarettes of flue-cured tobacco leaves; Agr. Biol. Chem. 42 (1978) 1527-1531.

176. Ishiguro, S. and S. Sugawara: The chemistry of tobacco smoke; Sci. Papers, Cent. Res. Inst., Japan Monopoly Corp. 298 (1980) 1-248.

177. Izawa, M.: Review of studies of tobacco smoke; Nippon Senbai Kosha (1961).

178. Jenkins, R.W. Jr: A review of the uses of nuclear radiation in tobacco and smoke research; Beitr. Tabakforsch. Int. 14 (1990) 353-378.

179. Jenkins, R.W. Jr, R.H. Newman, M.D. Edmonds, and T.S. Osdene: Dotriacontane - A precursor to benzo[ $[a]$ pyrene in smoke? $26^{\text {th }}$ Tobacco Chemists' Research Conference, Program Booklet and Abstracts, Vol. 26, Paper No. 8, 1972, pp. 12-13; Cigarette smoke formation studies. III. The contribution of dotriacontane to the benzo[a]pyrene content of smoke; Beitr. Tabakforsch. 7 (1973) 154-157.

180. Johnson, R.R. and E.D. Alford: Aromatic hydrocarbons from mildly heated tobacco; $21^{\text {st }}$ Tobacco Chemists' Research Conference, Program Booklet and Abstracts, Vol. 21, Paper No. 15, 1967, p. 9.

180a. Johnson, W.R.: Benzo[a]pyrene yield from cigarettes containing aluminum; Memorandum to R.B. Seligman, August 12, 1965, see www.pmdocs.com 1003030249.

181. Johnson, W.R., R.W. Hale, J.W. Nedlock, and H.J. Grubbs: The distribution of products between mainstream and sidestream smoke; $26^{\text {th }}$ Tobacco Chemists' Research Conference, Program Booklet and Abstracts, Vol. 26, Paper No. 3, 1972, pp. 4-5; Johnson, W.R., R.W. Hale, J.W. Nedlock, H.J. Grubbs, and D.H. Powell: The distribution of products between mainstream and sidestream smoke; Tob. Sci. 17 (1973) 141-144.

182. Johnstone, R.A.W. and J.R. Plimmer: The chemical constituents of tobacco and tobacco smoke; Chem. Rev. 59 (1959) 885-936.

183. Johnstone, R.A.W. and P.M. Quan: Naphthalenes in cigarette smoke; Nature 199 (1963) 1184.

184. Kakhiani, Z.H.: Cancerogenic action of tobacco tar; Voprosy Onkol. 1 (1955) 96-100.

185. Kalaitzoglou, M. and C. Samara: Distribution of polycyclic aromatic hydrocarbons between the particulate and gas phase of mainstream cigarette smoke in relation to cigarette technological characteristics; 21 (2005) 331-344.

186. Kennaway, E.L. and I. Hieger: Carcinogenic substances and their fluorescent spectra; Brit. Med. J. 1930 (i) 1044-1046.

187. Kiryu, S. and M.P. Kuratsune: Polycyclic aromatic hydrocarbons in the cigarette tar produced by human smoking; Gann 57 (1966) 317-323.

188. Klimisch, H.-J.: Clean-up and separation methods for the analysis of polycyclic aromatic hydrocarbons. Fractionation of cigarette smoke condensate; Z. Anal. Chem. 264 (1973) 275-278.

189. Klimisch, H.-J.: Separation analysis of polycyclic aromatic hydrocarbons by high pressure liquid chromatography. Selective separation system for the quantitative estimation of isomeric benzpyrenes and coronene; J. Chromatog. 83 (1973) 11-14.

190. Klimisch, H.-J. and D. Ambrosius: Quantitative determination of benzo $[a]$ pyrene in cigarette smoke condensates by high pressure liquid chromatography; Z. Anal. Chem. 280 (1976) 377-379.

191. Klimisch, H.-J. and E. Kirchheim: A rapid method for the determination of benzo $[a]$ pyrene, benz $[a]$ anthracene, and chrysene in cigarette smoke; Chromatographia 9 (1976) 119-122.

192. Klus, H. and H. Kuhn: Verteilung verschiedener Tabakrauchbestandteile auf Haupt- und Nebenstromrauch (Eine Übersicht) [Distribution of various tobacco smoke components among mainstream and sidestream smoke (A survey)]; Beitr. Tabakforsch. Int. 11 (1982) 229-265.

193. Koiwai, A., K. Nishida, M. Noguchi, and K. Arima: Studies on the fermentation of tobacco. Part II - A study of variations in fermentation procedure and its effect on total particulate matter and benzo $[a]$ pyrene; Tob. Sci. 15 (1971) 108-110.

194. Kosak, A.I.: The composition of tobacco smoke; Experientia 10 (1954) 69-71.

195. Kosak, A.I.: Chemistry of tobacco smoke; Chapter I, in: Biologic effects of tobacco, edited by E.L. Wynder, Little, Brown and Company, Boston, MA, 1955.

196. Kröller, E.: Ergebnisse vergleichender Schwel- und Rauchversuche an Tabak [Comparative results of smoldering and smoking studies on tobacco]; Deut. Lebensm. Rundschau 60 (1964) 214-215.

197. Kröller, E.: Zur Untersuchung der Schwelprodukte von Tabakzusatzstoffen [Investigation of the smoldering products of tobacco additives]; Lebensm. Chem. Gerichtl. Chem. 61 (1965) 234-237.

198. Kröller, E.: Zur Untersuchung der Pyrolyseprodukte von Tabakzusatzstoffen als Grundlage ihrer gesundheitlichen Beurteilung [Investigation of the pyrolysis products of tobacco additives as the basis of their health evaluation]; Z. Anal. Chem. 212 (1965) 46-53.

199. Kröller, E., The fluorometric determination of 3,4benzpyrene in low-temperature carbonization and smoke condensates; Bundesgesundheitsblatt 9 (1966) 36-67.

200. Kröller, E.: Ergebnisse von Schwelversuchen an Farbstoffen zur Farbmattierung von Tabakwaren. 5. Mitteilung. (Gelbholzextrakt) [Results of smoldering tests on dyes for the color matt finish of tobacco products. 5 . Report. (Fustic extracts)]; Bundesgesundheitsblatt 9 (1966) 173-174.

201. Kröller, E.: Untersuchungen zum Nachweis von Nitros- 
aminen in Tabakrauch und Lebensmitteln [Study of the determination of nitrosamines in tobacco smoke and food]; Deut. Lebensm. Rundschau 63 (1967) 303-305.

202. Kuhn, H.: Tobacco alkaloids and their pyrolysis products in the smoke; Tobacco Alkaloid Symp., Stockholm, Sweden (1964) 37-51; Tabakalkaloide und ihre Pyrolyseprodukte im Tabakrauch [Tobacco alkaloids and their pyrolysis products in smoke]; Fachliche Mitt. Österr. Tabakregie 1964 (5) 73-82.

203. Kuratsune, M.: Benzo[a]pyrene content of certain pyrogenic materials; J. Natl. Cancer Inst. 16 (1956) 1485-1496.

204. Lacassagne, A., N.P. Buu-Hoï, R. Daudel, and F. Zajdela: The relation between carcinogenic activity and the physical and chemical properties of angular benzacridines; Adv. Cancer Res. 4 (1956) 316-369.

205. Lacassagne, A., N.P. Buu-Hoï, and G. Rudall: Inhibition of the carcinogenic action produced by a weakly carcinogenic hydrocarbon on a highly active hydrocarbon; Brit. J. Exptl. Path. 26 (1945) 5-12.

206. Lacassagne, A., N.P. Buu-Hoï, and F. Zajdela: Activité cancérigène d'hydrocarbures polycycliques derivés du naphtacène [Carcinogenic activity of hydrocarbons derived from naphthacene]; Compt. Rend. 250 (1960) 3547-3548.

207. Lacassagne, A., N.P. Buu-Hoï, F. Zajdela, and F.A. Vingiello: The true dibenzo[ $a, l]$ pyrene, a new, potent carcinogen; Naturwissenschaften 55 (1968) 43.

208. Lakritz, L., R.L. Stedman, E.D. Strange, and D.G. Bailey: Attempts to alter the reducing properties and composition of cigarette smoke by the use of additives; $24^{\text {th }}$ Tobacco Chemists' Research Conference, Program Booklet and Abstracts, Vol. 24, Paper No. 23, 1970, p. 14; Lakritz, L., E.D. Strange, D.G. Bailey, and R.L. Stedman: Composition studies on tobacco. XLV. Use of tobacco additives to alter the composition and reducing properties of cigarette smoke; Beitr. Tabakforsch. 6 (1972) 120-123.

209. Lam, J.: 3,4-Benzopyrene as a product of the pyrolysis of aliphatic hydrocarbons; Acta Path. Microbiol. Scand. 37 (1955) 421-428; Isolation and identification of 3,4benzopyrene, chrysene, and a number of other aromatic hydrocarbons in the pyrolysis products of dicetyl; Acta Path. Microbiol. Scand. 39 (1956) 198-206; Determination of 3,4-benzopyrene and other aromatic hydrocarbons formed by pyrolysis of aliphatic tobacco hydrocarbons; Acta Path. Microbiol. Scand. 39 (1956) 207-210.

210. Latarjet, R., J.L. Cuzin, M. Hubert-Habart, B. Muel, and R. Royer: Détection quantitative du 3,4 benzopyrène formé par combustion du papier à cigarettes et du tabac [Quantitative determination of 3,4-benzpyrene formed by combustion of cigarette paper and of tobacco]; Bull. Assoc. France Étude Cancer 43 (1958) 180-198.

211. Latimer, P.H.: Compounds isolated from tobacco and smoke; RDM, 1955, No. 47, October 28, see www.rjrtdocs.com $500610337-0357$.

212. Latimer, P.H. and A. Rodgman: Compounds reported in tobacco smoke; Memorandum, September, 1957, see www.rjrtdocs.com $503015383-5387$.

212a. Laurene, A.H.: The composition of the vapor phase of cigarette smoke; RDR, 1955, No. 11, October 17, see www.rjrtdocs.com $501663325-3341$.

213. Lavit-Lamy, D. and N.P. Buu-Hoï: The true nature of "dibenzo $[a, l]$ pyrene" and its known derivatives; Chem. Comm. 4 (1966) 92-94.

214. Lazar, P.H., I. Chouroulinkov, C. Libermann, and M. Guerin: Amounts of 3,4-benzpyrene (3,4-BP) in cigarette smoke condensates and carcinogenicity; $9^{\text {th }}$ Internat. Cancer Cong., Tokyo, Japan (1966); Benzo[a]pyrene content and carcinogenicity of cigarette smoke condensate: Results of short-term and long-term tests; J. Natl. Cancer Inst. 37 (1966) 573-579.

215. Ledford, C.J., G.P. Morie, and C.A. Glover: Separation and determination of polynuclear aromatic hydrocarbons in cigarette smoke by high resolution liquid chromatography; $23^{\text {rd }}$ Tobacco Chemists' Research Conference, Program Booklet and Abstracts, Vol. 23, Paper No. 11, 1969, p. 8; Separation of polynuclear aromatic hydrocarbons in cigarette smoke by highresolution liquid chromatography; Tob. Sci. 14 (1969) 158-160; Anal. Chem. 47 (1975) 1155-1157.

216. Lee, M.L., M. Novotny, and K.D. Bartle: Gas chromatography/mass spectrometric and nuclear magnetic resonance spectrometric studies of carcinogenic polynuclear aromatic hydrocarbons in tobacco and marijuana smoke condensate; Anal. Chem. 48 (1976) 405-416.

217. Lefemine, D.V., E.T. Alvord, and S.Z. Cardon: Identification of 3,4-benzpyrene in cigarette paper smoke and tars; Southeastern Reg. Mtg., Am. Chem. Soc., Birmingham, AL, 1954; see Rodgman A.: RDM, 1954, No. 26, November 5, see www.rjrtdocs.com $504913137-3140$.

218. Leffingwell, J.C. and G. Worrell: Evaluation of $\mathrm{C}_{11}-\mathrm{C}_{18}$ hydrocarbons in filter tow for benzopyrene removal (Chemfilt Corporation of America); RDM, 1972, No. 8, February 9, see www.rjrtdocs.com 500615356-5360.

219. Lettré, H. and A. Jahn: Zur Bildung aromatischer Kohlenwasserstoffe während des Rauchprozesses [On the formation of aromatic hydrocarbons during the smoking process]; Naturwissenschaften 42 (1955) 210.

220. Lettré, H., A. Jahn, and C. Hausbeck: Nachweis von 3,4-Benzpyrene unter den Rauchprodukten [Detection of 3,4-benzpyrene in smoke products]; Angew. Chem. 68 (1956) 212-213.

221. Lindsey, A.J.: The composition of tobacco smoke. Some minor organic constituents; Analyst 80 (1955) 164.

222. Lindsey, A.J.: Tobacco smoke; Brit. Med. J. 1959 (ii) 506.

223. Lindsey, A.J.: The composition of cigarette smoke: Studies on stubs and tips; Brit. J. Cancer 13 (1959) 195-199.

224. Lindsey, A.J.: Some observations on the chemistry of tobacco smoke; in: Tobacco and health, edited by G. James and T. Rosenthal, Springfield, MA, 1962, pp. 21-32.

225. Lindsey, A.J., K. Persaud, and A. Candeli: Reduction of benzpyrene in tobacco smoke; Brit. Med. J. 1959 (ii) 821.

226. Lipp, G.: Über die Zusammensetzung des Cigarettenrauches und ihre Abhängigkeit von der Tabaksorte [Cigarette smoke composition and its dependence on tobacco type]; Beitr. Tabakforsch. 3 (1965) 1-13.

227. Lyons, M.J.: Tobacco smoke products. Assay for polycyclic hydrocarbons; Brit. Emp. Cancer Camp., Ann. Rpt. 33 (1955) 277-278; Assay of possible carcinogenic hydrocarbons from cigarette smoke; Nature 177 
(1956) 630-631.

228. Lyons, M.J.: Presence of 1,2,3,4-dibenzpyrene in cigarette smoke; Nature 182 (1958) 178.

229. Lyons, M.J.: Comparison of aromatic polycyclic hydrocarbons from gasoline engine and diesel engine exhausts, general atmospheric dust, and cigarette smoke condensate; in: Symposium: Analysis of carcinogenic air pollutants, edited by E. Sawicki and K. Cassels Jr, Natl. Cancer Inst. Monograph 9 (1962) 193-199.

230. Lyons, M.J. and H. Johnston: Chemical analysis of the neutral fraction of cigarettes smoke tar; Brit. J. Cancer 11 (1957) 554-562.

231. Macias, M.Y., W.C. Herndon, and I. Agranat: Thermodynamic properties of the arene epoxides and the relative carcinogenicities of benzo $[a]$ pyrene and benzo[e]pyrene; Polycyclic Aromatic Compounds 3 (1993) 199-207.

232. Maga, J.A.: Potential health concerns associated with smoke; in: Smoke in food processing, by J.A. Maga, CRC Press Inc., Boca Raton, FL, 1988, pp. 113-144.

233. Magee, P.N.: Natur, Entstehung und Vorkommen alkylierend wirkender Substanzen in Tabak und Tabakrauch [The nature, formation and occurrence of alkylating substances in tobacco and tobacco smoke]; in: Compounds having alkylating action, Verband der Cigarettenindustrie, Wissenschaftliche Forschungstelle (1964) 121-130.

233a. Magee, P.N. and J.M. Barnes: The production of malignant hepatic tumours in the rat by feeding with dimethylnitrosamine; Brit. J. Cancer 10 (1956) 114-122.

234. Martin, J.M. and J.T. Dobbins Jr: Gas chromatography of polynuclear aromatic hydrocarbons; RDM, 1968, No. 63, October 17, see www.rjrtdocs.com 500604315 -4323; Progress report. RDM, 1970, No. 1, January 7, see www.rjitdocs.com $500614167-4175$.

234a. Martin, P., C.J. Smith, R. Garg, G.A. Long, and C. Hansch: Molecular parameters reported for a series of polycyclic aromatic hydrocarbons (PAHs); 58 ${ }^{\text {th }}$ Tobacco Science Research Conference, Program Booklet and Abstracts, Vol. 58, Paper No. 43, 2004, p. 47.

235. Matthews, J.E.: An investigation of certain factors affecting the composition of cigarette smoke; Thesis, Pennsylvania State College (1941) pp. 1-77.

236. Matthey, E.: Efficacité du traitement préalable du tabac par un solvent organique, du point de vue de la réduction des substances toxiques dans la fumée [Effectiveness of the preliminary treatment of tobacco with an organic solvent from the point of view of reduction of toxic substances in the smoke]; $\mathrm{Z}$. Präventivmed. 6 (1961) 428-443.

237. Mauldin, R.K.: The characterization of cigarette smoke from Cytrel $®$ smoking product and its comparison to smoke from flue-cured tobacco. II. Semi-volatile vapor phase analysis; Beitr. Tabakforsch. 8 (1976) 422-429.

238. Michelson, I. and G. Rathkamp: Composition of cigarette smoke: Effects of ammonium sulfamate in cigarette paper; Beitr. Tabakforsch. 7 (1974) 212-216.

239. Miller, R.L., W.L. Chamberlain, and R.L. Stedman: Composition studies on tobacco. XXXIV. Pilot investigations on a concentrated polynuclear hydrocarbon fraction of smoke condensate; Tob. Sci. 13 (1969) 21.

239a. Mold, J.D., T.B. Walker, and L.G. Veasey: Selective separation of polycyclic aromatic compounds by countercurrent distribution with a solvent system con- taining tetramethyluric acid; Anal. Chem. 35 (1963) 2071-2074.

240. Morie, G.P., C.H. Sloan, and M.S. Baggett: Parameters affecting the selective filtration of certain tobacco smoke components; CORESTA 1974 Symp., Montreux, Switzerland, 1974; Beitr. Tabakforsch. 8 (1974) 145-149.

241. Mouron, J.C., J. Bonnet, and S. Neukomm: Extraction of tobacco by some organic solvents and consequences on chemical composition of the smoke; Oncologia 13 (1960) 271-278.

242. Muel, B. and G. LaCroix: Caractérisation et dosage du 3-4 benzopyrène par spectrophotométrie de luminescence à $-190{ }^{\circ} \mathrm{C}$ [Characterization and level of 3,4benzpyrene by luminescence spectrophotometry at -190 $\left.{ }^{\circ} \mathrm{C}\right]$; Bull. Soc. Chim. France (1960) 2139-2147.

243. Müller, K.H., G. Neurath, and H. Horstmann: Effect of air permeability of cigarette paper on the yield and composition of smoke condensate; Beitr. Tabakforsch. 2 (1964) 271-281.

244. Müller R, W. Moldenhauer, and P. Schlemmer: Erfahrung bei der quantitativen Bestimmung von polyzyklischen Kohlenwasserstoffen im Tabakrauch [Knowledge about of the quantitative estimation of polycyclic aromatic hydrocarbons in tobacco smoke]; Ber. Inst. Tabakforsch. Dresden 14 (1967) 159.

245. National Cancer Institute: Report No. 5. Toward less hazardous cigarettes. Summary: Skin painting bioassays using condensate from experimental cigarettes; DHEW Publ. (NIH) (September, 1980).

246. Neukomm, S.: Experimental studies on the carcinogenic power of tobacco smoke and other contaminants of the atmosphere; Oncologia 10 (1957) 137-155.

247. Neukomm, S.: The newt test in relation to investigations on carcinogenic substances; in: Symposium: Analysis of carcinogenic air pollutants, edited by E. Sawicki and K. Cassels Jr, J. Natl. Cancer Inst. Monograph 9 (1962) 71-73.

248. Neukomm, S.: Cocarcinogenic action of various fractions of tobacco smoke; Acta Unio Internat. Contra Cancrum 18 (1962) 33-36.

249. Neukomm, S. and J. Bonnet: Untersuchungen über cancerogene Stoffe im Tabakrauch [Investigation of the carcinogenic compounds in tobacco smoke]; StrahlenTherapie 37 (Suppl.) (1957) 128-132.

250. Neukomm, S. and J. Bonnet: Carcinogenic and cocarcinogenic substances in tobacco smoke; $7^{\text {th }}$ Internat. Cancer Cong., London, England, 1958.

251. Neukomm, S. and J. Bonnet: Perfectionnements dans le traitement du tabac [Improvements in the treatment of tobacco]; French Patent No. 1,205,390 (February 2, 1960).

252. Neukomm, S. and J. Bonnet: On the combustion of organic material and origin of carcinogenic substances in tobacco and in food; Oncologia 13 (1960) 266-271.

253. Neukomm, S. and J. Bonnet: Process for treating tobacco and tobacco obtained by said process; U.S. Patent No. 3,096,773 (July 9, 1963).

254. Neurath, G.: Piègeage de la fumée secondaire de la cigarette [Trapping sidestream cigarette smoke]; CORESTA Inf. Bull. 1964 (2) 314.

255. Neurath, G.: Tobacco products and smoke; Proc. $4^{\text {th }}$ Internat. Tob. Sci. Cong., Athens, Greece, 1966 (1967) 743-760; Tobacco products and smoke. General report; Beitr. Tabakforsch. 4 (1967) 1-17. 
256. Neurath, G.: Stickstoffverbindungen des Tabakrauches [Nitrogen compounds of tobacco smoke]; Arzneimittelforschung 19 (1969) 1093-1106; Beitr. Tabakforsch. 5 (1969) 115-133.

257. Neurath, G., M. Dünger, and I. Küstermann: Untersuchungen der "Semivolatiles" des Cigarettenrauches [Study of the "semivolatiles" in cigarette smoke]; Beitr. Tabakforsch. 6 (1971) 12-20.

258. Neurath, G. and H. Ehmke: Apparatur zur Untersuchung des Nebenstromrauches [Apparatus to study sidestream smoke]; Beitr. Tabakforsch. 2 (1964) 117-121.

259. Neurath, G. and H. Ehmke: Untersuchungen über den Nitratgehalt des Tabaks [Study of the nitrate content of tobacco]; Beitr. Tabakforsch. 2 (1964) 333-334.

260. Neurath, G., Gewe J, and H. Wichern: Über das Vorkommen von Benzofuranen im Tabakrauch [On the occurrence of benzofurans in tobacco smoke]; Beitr. Tabakforsch. 4 (1968) 247-249.

261. Neurath, G., Gewe J, and H. Wichern: Über das Vorkommen von Hydroaromaten im Tabakrauch [On the occurrence of hydroaromatics in tobacco smoke]; Beitr. Tabakforsch. 4 (1968) 250-252.

262. Neurath, G. and H. Horstmann: Einfluss des Feuchtigskeitsgehaltes von Cigaretten auf die Zusammensetzung des Rauches und die Glutzonentemperatur [Effect of the moisture content of a cigarette on the composition of smoke and the burning zone temperature]; Beitr. Tabakforsch. 2 (1963) 93-100.

263. Newell, M.P., R.A. Heckman, and C.R. Green: Smoke composition: Homogenized G7L versus nonhomogenized tobacco blend; RDR, 1974, No. 4, April 30, see www.rjrtdocs.com 501003374 -3436.

264. Newell, M.P., R.A. Heckman, R.F. Moates, C.R. Green, F.W. Best, and J.N Schumacher: The composition of the ether-soluble portion of the particulate phase of cigarette smoke; $29^{\text {th }}$ Tobacco Chemists' Research Conference, Program Booklet and Abstracts, Vol. 29, Paper No. 39, 1975, p. 28; Isolation and identification of new components of the ether-soluble portion of cigarette smoke condensate; Tob. Sci. 22 (1978) 6-11.

265. Nicolaus, G. and H. Elmenhorst: Nachweis und quantitative Bestimmung von Alkylnaphthalinen in LatakiaTabak [Detection and quantitative estimation of the alkylnaphthalenes in Latakia tobacco]; Beitr. Tabakforsch. Int. 11 (1982) 133-140.

265a. Oakley, E.T.: Benzo[a]pyrene delivery of all-burley, all-bright, and all-Turkish cigarettes; Memorandum to F.E. Resnik, February 28, 1966, see www.pmdocs.com $1001884565 / 4566$.

265b.Oakley, E.T.: Benzo[a]pyrene in sidestream smoke; Memorandum to F.E. Resnik, February 1, 1966, see www.pmdocs.com 1000702902.

266. Oakley, E.T., L.F. Johnson, and H.M. Stahr: A rapid method for the determination of benzo[a]pyrene in cigarette smoke; Tob. Sci. 16 (1972) 19-21.

266a. Oakley, E.T. and H.M. Stahr: Benzo $[a]$ pyrene in cigarette smoke: Method No. S-23; Report, October 1965, see www.pmdocs.com 2504108144/8150.

267. Obi, Y., M. Muramatsu, and Y. Shimada: Quality coefficient of tobacco leaves by gas phase of main stream smoke of cigarette; Tob. Sci. 12 (1968) $161-162$

268. Occupational Safety and Health Administration
(OSHA): Indoor air quality; Fed. Reg. 59 (No. 65) (1994) 15968-16039.

269. Oliver, B.W.: Thin-layer chromatographic separation of benzo[a]pyrene from cigarette tar; $19^{\text {th }}$ Tobacco Chemists' Research Conference, Program Booklet and Abstracts, Vol. 19, Paper No. 35, 1965, pp. 51-53.

270. Onishi, I.: Recent studies on tobacco smoke by the Japan Monopoly Corporation; Proc. $2^{\text {nd }}$ Internat. Tob. Sci. Cong., Brussels, Belgium (1959) 553-559.

271. Orris L, B.L. Van Duuren, A.I. Kosak, N. Nelson, and F.L. Schmitt: The carcinogenicity for mouse skin and the aromatic hydrocarbon content of cigarette-smoke condensate; J. Natl. Cancer Inst. 21 (1958) 557-561.

272. Pailer, M., W. Hübsch, and H. Kuhn: Beitrag zur Bestimmung des Benzo[a]pyrens in Tabakrauchkondensaten [Contribution to the determination of benzo[a]pyrene in tobacco smoke condensate]]; Fachl. Mitt. Österr. Tabakregie, Spec. Issue (1965) i-xi.

273. Pappas, N.A. and X.E. Binopoulos: Isolation, identification and determination of polycyclic aromatic hydrocarbons of cigarette smoke condensate using the thin layer chromatographic technique. Proc. $4^{\text {th }}$ Internat. Tob. Sci. Cong., Athens, Greece, 1966 (1967) 979-1002.

274. Parmele, H.B. and C.O. Jensen: Smoking tobacco product; British Patent No. 903,067 (August 9, 1962).

275. Patel, A.R., M.Z. Haq, C.L. Innerarity, L.T. Innerarity, and K. Weissgraber; Fractionation studies of smoke condensate from Kentucky Reference Cigarettes; Tob. Sci. 19 (1974) 58-59.

275a. Pauling, L.: Nature of the chemical bond; Cornell University Press, Ithaca, NY, 1937.

276. Pavlu, J. and J. Sula: Detection and estimation of 3,4benzpyrene and arsenic in cigarette smoke; Casopis Lekaru Ceskych 96 (1960) 101-104.

277. Perakis, X.: Contribution to the determination of 3,4benzpyrene in tobacco smoke condensate; Z. Anal. Chem. 204 (1964) 28-32.

278. Philip Morris Inc.: Chemical constituents in tobacco and smoke. A compilation of published information; Philip Morris Inc., Richmond, VA, 1963, pp. 1-47.

279. Pietzsch, A.: Die Papierchromatographie cancerogener Kohlenwasserstoffe [Paper chromatography of carcinogenic hydrocarbons]; Pharmazie (Berlin) 12 (1957) 24-30.

280. Pietzsch, A.: Zum Nachweis von cancerogenen Kohlenwasserstoffen im Tabakrauch [On the detection of carcinogenic hydrocarbons in tobacco smoke]; Naturwissenschaften 45 (1958) 445; Pharmazie (Berlin) 14 (1959) 466-473.

281. Pietzsch, A.: Ein weiterer Beitrag zum Thema Retinierung von Rauchbestandteilen beim Zigarettenrauchen [Another contribution to the reduction of smoke components in cigarette smoke]; Pharmazie (Berlin) 17 (1962) 36-41.

282. Pietzsch, A.: Demonstration of carcinogenic hydrocarbons, with special reference to paper chromatography; Arch. Geschwulstforsch. 21 (1964) 137-143, see Carc. Abstr. 2 (2) (1964) 64-245.

282a. Poel, W.E.: Carcinogens and minimal carcinogenic doses; Science 123 (1956) 588; Poel, W.E., D. Stanton, E. Peters, and H.O. Wade: Approximation of a threshold-maximal carcinogenic dose range for 3,4benzpyrene when applied repeatedly to mouse skin; Proc. Am. Assoc. Cancer Res. 4 (2) (1958) 333; Poel, 
W.E. and A.G. Kammer: Preliminary studies in a quantitative approach to skin carcinogenesis; J. Natl. Cancer Inst. 16 (1958) 989-994.

282b.Preussmann, R. and B.W. Stewart: $N$-Nitroso carcinogens; Chapter 12, in: Chemical Carcinogens. Second Edition, edited by C.E. Searle, American Chemical Society Monograph 182, American Chemical Society, Washington, DC, 1984, pp. 643-828.

283. Pullman, A. and B. Pullman: Electronic structure and carcinogenic activity of aromatic molecules. New developments; Adv. Cancer Res. 3 (1955) 117-169.

284. Purkis, S.W., C.A. Hill, and I.A. Bailey: Current reliability of measurements of smoke analytes; $55^{\text {th }}$ Tobacco Science Research Conference, Program Booklet and Abstracts, Vol. 55, Paper No. 16, 2001, pp. 29-30; Current measurement reliability of selected smoke analytes; Beitr. Tabakforsch. Int. 20 (2003) 314-324.

285. Pyriki, C.: Polycyclische Kohlenwasserstoffe im Zigarettenrauch [Polycyclic hydrocarbons in cigarette smoke]; Mitt. Gdch-Fachgruppe Lebens. Gericht. Chem. 14 (2) (1960) 27-29.

286. Pyriki, C.: Polycyclische und aliphatische Kohlenwasserstoffe des Tabakrauches [Polycyclic and aliphatic hydrocarbons in cigarette smoke]; German Chem. Soc., Ann. Mtg., Leipzig DDR (1962); Die Nahrung 7 (1963) 439-448.

287. Pyriki, C., W. Moldenauer, and T.W. Knappe: Zur Frage der Behandlung des Zigarettenpapieres und des Tabaks zwecks Verminderung von polycyclischen Kohlenwasserstoffen in deren Rauch [On the question of treatment of cigarette paper and the tobacco in order to reduce the polycyclic hydrocarbons in its smoke]; Ber. Inst. Tabakforsch. Dresden 12 (1965) 37-55.

288. Pyriki, C., R. Müller, and W. Moldenauer: Über das Auftreten von polycyclischen Kohlenwasserstoffen im Zigarettenrauch. II. Untersuchung des lipophilen Anteiles der einzelnen Rauchphasen sowie des Tabaks [On the occurrence of polycyclic hydrocarbons in cigarette smoke. II. Study of the lipophilic components in the separate smoke phases as well as tobacco]; Ber. Inst. Tabakforsch. Dresden 7 (1960) 81-102.

289. Quin, L.D.: Chemical studies on tobacco and its smoke; Selecta Chim. 2 (1962) 37-62.

290. Rand, H.J., E.T. Alvord, S.Z. Cardon, and A. Burhan: A study of cigarette smoke and cigarette paper smoke alone; Am. J. Surg. 94 (1957) 438-443.

291. Rand, H.J., S.Z. Cardon, E.T. Alvord, and A. Burhan: A study of cigarette smoke and cigarette paper smoke alone; Cancer Cytol. 4 (1961) 18-21.

292. Rathkamp, G. and D. Hoffmann: The inhibition of the pyrosynthesis of several selective smoke components. Experimental findings and theoretical considerations; $23^{\text {rd }}$ Tobacco Chemists' Research Conference, Program Booklet and Abstracts, Vol. 23, Paper No. 29, 1969, p. 21.

293. Rathkamp, G. and D. Hoffmann: Chemical studies on tobacco smoke. XIII. Inhibition of the pyrosynthesis of several selective smoke constituents; Beitr. Tabakforsch. 5 (1970) 302-306.

294. Rathkamp, G. and D. Hoffmann: Fluorenes and fluoranthenes in cigarette smoke; $24^{\text {th }}$ Tobacco Chemists' Research Conference, Program Booklet and Abstracts, Vol. 24, Paper No. 28, 1970, p. 20.

295. Rathkamp, G. and D. Hoffmann: Polynuclear aromatic hydrocarbon profiles of tobacco smoke; $26^{\text {th }}$ Tobacco Chemists' Research Conference, Program Booklet and Abstracts, Vol. 26, Paper No. 9, 1972, p. 14.

296. Rathkamp, G., D. Hoffmann, and E.L. Wynder: Experiments on the reduction of polynuclear aromatic hydrocarbons in cigarette smoke; $20^{\text {th }}$ Tobacco Chemists' Research Conference, Program Booklet and Abstracts, Vol. 20, Paper No. 19, 1966, pp. 23-25.

297. Rathkamp, G., T.C. Tso, and D. Hoffmann: Chemical studies on tobacco smoke. XX. Smoke analyses of cigarettes made from bright tobacco differing in variety and stalk position; Beitr. Tabakforsch. 7 (1973) 179-189.

298. Rayburn, C.H., W.B. Wartman Jr, and P.M. Pedersen: Influence of hexane solubles in tobacco on a polycyclic fraction of cigarette smoke; $133^{\text {rd }}$ Natl. Mtg., Am. Chem. Soc., San Francisco, CA (April 15, 1958); Science 128 (1958) 1344-1345.

298a. Resnik, F.E. and J.C. Holmes: New techniques of smoke analysis. II. Mass spectrometric identification of smoke constituents from gas chromatography fractions; $9^{\text {th }}$ Tobacco Chemists' Research Conference, Program Booklet and Abstracts, Vol. 9, Paper No. 5, 1955, pp. 2-3.

299. Risner, C.H.: The determination of benzo $[a]$ pyrene and benz $[a]$ anthracene in the total particulate matter of cigarette smoke by high-performance liquid chromatography; R\&DM, 1986, No. 143, September 2, see www.rjitdocs.com 506472122 -2167; The determination of benzo $[a]$ pyrene in the total particulate matter of cigarette smoke; $40^{\text {th }}$ Tobacco Chemists' Research Conference, Program Booklet and Abstracts, Vol. 40, Paper No. 39, 1986, p. 21; The determination of benzo $[a]$ pyrene in the total particulate matter of cigarette smoke; J. Chromat. Sci. 26 (1988) 113-120.

300. Risner, C.H.: The determination of benzo $[a]$ pyrene and benz $[a]$ anthracene in mainstream and sidestream smoke of Reference Cigarette 1R4F and a cigarette which heats but does not burn tobacco: a comparison; R\&DM, 1988, No. 173, June 29, see www.rjrtdocs.com 512059871 -9894; Risner, C.H.: The determination of benzo $[a]$ pyrene and benz $[a]$ anthracene in mainstream and sidestream smoke of Kentucky Reference Cigarette 1R4F and a cigarette which heats but does not burn tobacco: A comparison; Beitr. Tabakforsch. Int. 15 (1991) 11-17.

301. R. J. Reynolds Tobacco Company: Chemical and biological studies: New cigarette prototypes that heat instead of burn tobacco; R. J. Reynolds Tobacco Company, Winston-Salem, NC, 1988.

301a. Robb, E.W., G.C. Guvernator III, M.D. Edmonds, and A. Bavley: Analysis of polycyclic hydrocarbons in cigarette smoke; Report, 1963, see www.pmdocs.com $1001895592 / 5611$.

302. Robb, E.W., G.C. Guvernator III, M.D. Edmonds, and A. Bavley: Improved methods for determination of benzo $[a]$ pyrene in cigarette smoke; CORESTA Sci. Comm. Mtg., Vienna, Austria (1964); Analysis of polycyclic hydrocarbons; Beitr. Tabakforsch. 3 (1965) 278-284.

303. Roberts, D.L., J.N. Schumacher, R.A. Lloyd, R.A. Heckman, and A. Rodgman: List of tobacco and smoke constituents; RDM, 1975, No. 15, April 16, see www.rjrtdocs.com 514901435 -1636.

304. Roberts, D.L., J.N. Schumacher, R.A. Lloyd, and R.A. 
Heckman: Literature study of pyrolysis of amino acids and proteins; RDM, 1976, No. 9, February 11, see www.rjrtdocs.com $500616651-6671$.

305. Rocchietta, S.: Recent research on the identification of 3,4-benzpyrene in the components of tobacco smoke; Minerva Med. (Torino) 47 (1956) 1831.

306. Rodgman, A.: The carcinogenicity of 3,4-benzpyrene; Memorandum, 18 October 1954, pp. 1-13.

307. Rodgman, A.: Data discussed by Dr. Ernst L. Wynder in speech "Human and experimental relationship of cancer and tobacco" Presented at the Symposium on "Tobacco" before the Metropolitan Long Island Subsection of the American Chemical Society, February 24, 1956; RDM, 1956, No. 9, March 16, see www.rjrtdocs.com 501009747 -9754.

308. Rodgman, A.: The analysis of cigarette smoke condensate. I. The isolation and/or identification of polycyclic aromatic hydrocarbons in Camel cigarette smoke; RDR, 1956, No. 9, September 28, see www.rjirtdocs.com 501008241 -8293.

309. Rodgman, A.: The analysis of cigarette smoke condensate. II. The pretreatment of Camel blend tobacco; RDR, 1956, No. 12, November 1, see www.rjrtdocs.com 501008294 -8336.

310. Rodgman, A.: The analysis of cigarette smoke condensate. III. Flue-cured tobacco; RDR, 1957, No. 4, March 14, see www.rjrtdocs.com 501008337 -8377.

311. Rodgman, A.: Tobacco tar fractionation and the pretreatment of tobacco: A conversation with Dr. George F Wright of the University of Toronto, Toronto, Ontario, Canada; RDM, 1957, No. 25, July 22, see www.rjirtdocs.com 500610651 -0656.

312. Rodgman, A.: The analysis of cigarette smoke condensate. IV. 3,4,8,9-Dibenzpyrene in Camel cigarette smoke condensate; RDR, 1957, No. 13, October 7, see www.rjrtdocs.com $501008378-8386$.

313. Rodgman, A.: Components reported in tobacco smoke; RDM, 1958, No. 32, April 17, see www.rjrtdocs.com $500610933-0997$.

314. Rodgman, A.: The analysis of cigarette smoke condensate. VI. The influence of solvent pretreatment of tobacco and other factors on the polycyclic hydrocarbon content of smoke condensate; RDR, 1959, No. 1, January 29, see www.rjrtdocs.com 501008529-8591.

315. Rodgman, A.: The analysis of cigarette smoke condensate. XIX. The determination of polycyclic aromatic hydrocarbons; RDR, 1961, No. 1, January 6, see www.rjitdocs.com 500935976 -5996.

316. Rodgman, A.: The analysis of cigarette smoke condensate. XXXIII. Polycyclic hydrocarbons in Lark cigarette smoke; RDM, 1963, No. 37, May 13, see www.rjrtdocs.com $500612598-2601$.

317. Rodgman, A.: The analysis of cigarette smoke condensate. XXXV. A summary of an eight-year study; RDR, 1964, No. 10, February 12, see www.rjrtdocs.com 501008855 -8928.

318. Rodgman, A.: G13-expanded tobacco and Freon $11 \circledR$; December, 1972, pp. 1-66, see www.rjitdocs.com 521189661 -9727; G13-expanded tobacco and Freon $11 \circledR .1^{\text {st }}$ Revision, February, 1974, pp. 1-77, see www.rjrtdocs.com 521189578-9660; G13-Expanded tobacco and Freon 11®, $2^{\text {nd }}$ Revision, October, 1977, pp. 1-152, see www.rjrtdocs.com 515991960 -2115; G13-expanded tobacco and Freon $11 \AA, 3^{\text {rd }}$ Revision. December, 1979, pp. 1-152, see www.rjrtdocs.com
$515979463-9566$.

319. Rodgman, A.: A comparison of the chemical, physical, and biological properties of cigarette mainstream smoke (MS), cigarette sidestream smoke (SS), and environmental tobacco smoke (ETS); Report submitted to the U. S. Environmental Protection Agency (December 1991; revised July 1992), pp. i-vii + 1-117, see www.rjrtdocs.com 508137542 -7573; 508185686 -5809 .

320. Rodgman, A.: The chemical composition of environmental tobacco smoke: Some comments on the Occupational Safety and Health Administration's notice on 'Indoor Air Quality'; Document submitted to the Occupational Safety and Health Administration, 5 August, 1994, pp. i-xiii + 1-172, see www.rjrtdocs.com 515923456 -3645; $517582702-2904$.

321. Rodgman, A.: Comments on BPDE experiments of Denissenko et al.; Personal communication, November 26, 1996, pp. 1-10.

321a. Rodgman, A.: The $N$-nitrosamines in tobacco and tobacco smoke; October, 1993: pp. i-xv, 1-259, see www.rjrtdocs.com 509752851 -3134.

322. Rodgman, A.: Tobacco smoke components. Letter to the Editor; Beitr. Tabakforsch. Int. 18 (1998) 127-129.

323. Rodgman, A.: Studies of polycyclic aromatic hydrocarbons in cigarette mainstream smoke: Identification, tobacco precursors, control of levels: A review; Beitr. Tabakforsch. Int. 19 (2001) 361-379.

324. Rodgman, A.: Some studies of the effects of additives on cigarette mainstream smoke properties. I. Flavorants; Beitr. Tabakforsch. Int. 20 (2002) 83-103.

325. Rodgman, A.: Some studies of the effects of additives on cigarette mainstream smoke properties. II. Casing materials and humectants; Beitr. Tabakforsch. Int. 20 (2002) 279-299.

326. Rodgman, A.: The composition of cigarette smoke: Problems with lists of tumorigens; Beitr. Tabakforsch. Int. 20 (2003) 402-437.

327. Rodgman, A. and L.C. Cook: The analysis of cigarette smoke condensate. V. The polycyclic hydrocarbon precursors in tobacco; RDR, 1958, No. 18, December 1, see www.rjitdocs.com 501008387 -8441.

328. Rodgman, A. and L.C. Cook: The analysis of cigarette smoke condensate. XIII. Sclareolide from Turkish tobacco smoke; RDR, 1960, No. 8, April 1, see www.rjrtdocs.com 500934533 -4541.

329. Rodgman, A. and L.C. Cook: The analysis of cigarette smoke condensate. XIV. Polycyclic aromatic hydrocarbons; RDR, 1960, No. 20, May 26, see www.rjrtdocs.com $501008592-8660$.

330. Rodgman, A. and L.C. Cook: The analysis of cigarette smoke condensate. XVII. The effect of aluminasupported catalysts on total polycyclic hydrocarbons; RDR, 1960, No. 36, December 2, see www.rjrtdocs.com 501008682 -8694.

331. Rodgman, A. and L.C. Cook: The analysis of cigarette smoke condensate. XVIII. Chloranil and 2,4,7trinitrofluorenone as filter tip additives; RDR, 1960, No. 38, December 7, see www.rjrtdocs.com 501008695 -8704 .

331a. Rodgman, A. and L.C. Cook: The analysis of cigarette smoke condensate. XXI. Phenols; RDR, 1961, No. 10, February 23, see www.rjrtdocs.com 501008731 -8772.

332. Rodgman, A. and L.C. Cook: The composition of cigarette smoke; Presented at Sigma Xi Meeting, Wake 
Forest University, Winston-Salem, NC, March 17, 1965, see www.rjrtdocs.com 501521599 -2606; the American Chemical Society Section Meeting, Columbus, GA, May 2, 1968, and the Chemistry Club, Chemistry Department, Columbus College, Columbus, GA, May 2 1968, see www.rjrtdocs.com 501521608 1625; and the Central North Carolina Section Meeting, American Chemical Society, Greensboro, NC, October 14, 1969, see www.rjrtdocs.com 501521658 -1700.

333. Rodgman, A. and L.C. Cook: The composition of cigarette smoke. Precursor studies; Unpublished manuscript, 1967, see www.rjrtdocs.com 501525257 5284, $521184403-4430$.

334. Rodgman, A. and L.C. Cook: The composition of cigarette smoke. Some minor components of the neutral-acidic fraction; Unpublished manuscript, 1967, see www.rjrtdocs.com 501525285 -5336, 521184431 $-4483$.

335. Rodgman, A., L.C. Cook, and S.S. Mims: The analysis of cigarette smoke condensate. XII. Squalenes and solanesenes; RDR, 1960, No. 3, February 10, see www.rjrtdocs.com 500934455-4473; The composition of cigarette smoke. V. Solanesenes; $14^{\text {th }}$ Tobacco Chemists' Research Conference, Program Booklet and Abstracts, Vol. 14, Paper No. 29, 1960, p. 15; J. Org. Chem. 26 (1961) 497-501, see www.rjrtdocs.com $521187398-7403$.

336. Rodgman, A. and C.R. Green: Toxic chemicals in cigarette mainstream smoke - hazard and hoopla; in: Cigarette risk and the potential for risk reduction; Proceedings of the 2002 CORESTA Congress, New Orleans LA, pp. 2-52; Beitr. Tabakforsch. Int. 20 (2003) 481-545.

336a. Rodgman, A., P. Martin, T.A. Perfetti, C.J. Smith, G.A. Long, and C. Hansch: Molecular parameters reported for a series of polycyclic aromatic hydrocarbons (PAHs). II; 59 ${ }^{\text {th }}$ Tobacco Science Research Conference, Program Booklet and Abstracts, Vol. 59, Paper No. 22, 2005, pp. 29-30.

337. Rodgman, A. and W.W. Menz: Components reported in tobacco smoke; RDM, 1960, No. 47, May 27, see www.rjrtdocs.com $500600177-0313$.

338. Rodgman, A., W.W. Menz, J.M. DeTombe, and G.A. Konstantinow: Components reported in tobacco smoke; RDM, 1965, No. 41, June 4, see www.rjrtdocs.com $500602714-3093$.

339. Rodgman, A., W.W. Menz, F.E. Huffmann, and E.N. Smith: Components reported in tobacco smoke; RDM, 1962, No. 43, May 21, see www.rjrtdocs.com $500601332-1501$.

340. Rodgman, A., W.W. Menz, and G.A. Konstantinow: Components reported in tobacco smoke. Supplement I. Components reported from May 1962 to April 1963; RDM, 1963, No. 32, May 2, see www.rjrtdocs.com $500612532-2587$.

341. Rodgman, A., C.J. Smith, and A.T. Perfetti: The composition of cigarette smoke: A retrospective, with emphasis on polycyclic components; Human Exptl. Toxicol. 19 (2000) 573-595, see www.rjrtdocs.com $524405137-5158$.

342. Rodgman, A. and B.W. Woosley: Components reported in tobacco smoke: A supplement to RDM, 1965, No. 41; RDM, 1967, No. 15, March 16, see www.rjrtdocs.com 500612947 -3178.

343. Roe, F.J.C.: The role of 3,4-benzopyrene in carcino- genesis by tobacco smoke condensate; Nature 194 (1962) 1089-1090.

344. Roe, F.J.C.: Role of 3,4-benzpyrene in carcinogenesis by tobacco smoke condensate; Acta Unio Internat. Contra Cancrum 19 (1963) 730.

345. Roffo, A.E.: Espectrograffe de los derivados obtenidos por destilacion directa de los tabacos y su relacion como agentes carcinogenos [Spectrometry of the compounds obtained by the destructive distillation of tobacco and the relation to carcinogenic agents]; Bol. Inst. Med. Exptl. Estud. Cancer 14 (1937) 311-399, see Chem. Abstr. 32 (1938) 381233 ; Bol. Inst. Med. Exptl. Estud. Cancer 17 (1940) 279.

346. Roffo, A.H.: Krebserzeugendes Benzpyren gewonnen aus Tabakteer [A cancer-causing benzpyrene isolated from tobacco tar]; Z. Krebsforsch. 49 (1939) 588-597, see Biol. Abstr. 14 (1939) 16122.

347. Roffo, A.H.: Krebserzeugende Einheit verschiedener Tabakteere [Cancer-causing properties of different tobacco tars]; Deut. Med. Wchnschr. 65 (1939) 963-967, see Chem. Abstr. 33 (1939) 94197; Unidad cancerígena de los alquitranes de diversos tipos de tabacos [Similar carcinogens in the tars from different tobacco types]; Bol. Inst. Med. Exptl. Estud. Cáncer 15 (1939) 349-406. See Chem. Abstr. 33 (1939) 5906; Prensa Méd. Argent. 26 (1939) 721-737, see Chem. Abstr. 34 (1940) $7120^{4}$.

348. Roffo, A.H.: 1,2-Benzopirene: Cancerigeno extraido del alquitran del tabaco [1,2-Benzpyrene: A carcinogen isolated from tobacco tar]; Bol. Inst. Med. Exp. Estud. Cancer 16 (1939) 1-38.

Note: In 1939, 1,2-benzopyrene was the original name assigned to 3,4-benzopyrene (64), subsequently renamed benzo[ $a]$ pyrene by IUPAC.

349. Roffo, A.H.: The principles of cancer production by tobacco; Schweiz. Med. Wchnschr. 71 (1941) 549-552.

350. Roffo, A.H.; El alquitrán de tabaco extraido y la disminución de cancerización [The tar from tobacco extracted to reduced carcinogenicity]; Bol. Inst. Med. Exptl. Estud. Cáncer 19 (1942) 431-502, see Chem. Abstr. 37 (1943) 6335.

351. Rothwell, K. and J.K. Whitehead: A method for the isolation of polycyclic aromatic hydrocarbons from complex hydrocarbon mixtures; Chem. and Ind. (London) (1967) 784-786.

352. Rothwell, K. and J.K. Whitehead: Complex formation, isolation and carcinogenicity of polycyclic aromatic hydrocarbons; Nature 213 (1967) 797.

353. Rothwell, K. and J.K. Whitehead: A method for the concentration of basic polycyclic heterocyclic compounds and the separation of polycyclic aromatic hydrocarbons from cigarette smoke condensate; Chem. and Ind. (London) (1969) 1628-1630.

354. Rothwell, K. and J.K. Whitehead: Fractionation of whole smoke condensate; $25^{\text {th }}$ Tobacco Chemists' Research Conference, Program Booklet and Abstracts, Vol. 25, Paper No. 30, 1971, p. 17.

355. Rowland, R.L., P.H. Latimer, and J.A. Giles: Fluecured tobacco. I. Isolation of solanesol, an unsaturated alcohol; J. Am. Chem. Soc. 78 (1957) 4680-4685; Tob. Sci. 1 (1957) 86-90.

356. Rubin, H.: Synergistic mechanisms in carcinogenesis by polycyclic hydrocarbons and by tobacco smoke: A bio-historical perspective with updates; Carcinogenesis 22 (2001) 1903-1930. 
357. Rustemeier, K., R. Stabbert, H.J. Haussmann, E. Roemer, and E.L. Carrnines: Evaluation of the potential effects of ingredients added to cigarettes. Part 2: Chemical composition of mainstream smoke: Food Chem. Toxicol. 40 (2002) 93-104.

358. Safaev, R., D.G. Zaridze, G.A. Belitskii, M.V. Djordjevic, D. Hoffmann, K.D. Brunnemann, Y.A. Perezhogina, N.N. Sokolskaya, A. Goginasvili, and Y. Khesina: Carcinogenic substances in the tobacco and smoke of cigarettes: Polynuclear aromatic hydrocarbons, metals, pesticides; Eksp. Onkol. 14 (3) (1992) 25-29.

359. Safaev, R., D.G. Zaridze, D. Hoffmann, K.D. Brunnemann, and Y. Liu: Efficiency and assessment of new cigarette filters. Chemical analysis of some of the toxic and carcinogenic agents in the mainstream smoke; Eksp. Onkol. 17 (1) (1995) 71-76.

360. Sakuma, H., M. Kusama, K. Yamaguchi, T. Matsuki, and S. Sugawara: Sidestream (SS)/mainstream (MS) distribution ratios of cigarette smoke components. III. Medium and high boiling components; $37^{\text {th }}$ Tobacco Chemists' Research Conference, Program Booklet and Abstracts, Vol. 37, Paper No. 41, 1983, p.23; Sakuma, H., M. Kusama, K. Yamaguchi, and S. Sugawara: The distribution of cigarette smoke components between mainstream and sidestream smoke. III. Middle and high boiling components; Beitr. Tabakforsch. Int. 12 (1984) 251-258.

361. Sanders, N.C., C.W. Miller, F.W. Best, A.L. Angel, and M.P. Newell: Comparative smoke studies. VII. Mainstream vs. sidestream "Winston monitor"; RDR, 1978, No. 6, December 29, see www.rjrtdocs.com 501005524 -5550 .

362. Sasaki, T.A. and S.C. Moldoveanu: Analysis of dibenz $[a, j]$ acridine in particulate-phase cigarette smoke; 53 ${ }^{\text {rd }}$ Tob. Sci. Res. Conf., Montreal PQ, Canada, Paper No. 32, p. 37 (1999); Determination of dibenz $[a, j]$ acridine in the particulate phase of cigarette smoke; Beitr. Tabakforsch. Int. 19 (2000) 25-31.

363. Sasmoco, A.: Improvements in or relating to a process for treating tobacco and tobacco obtained by said process; British Patent No. 885,249 (Cl. 130) (December 30, 1961).

364. Scassellati-Sforzolini, G. and A. Mariani: Benzopirene e di altri idrocarburi policiclici nel fumo delle sigarette "Nationale Esportazione" [Benzopyrene and other polycyclic hydrocarbons in the smoke of "Nationale Esportazione"]; Boll. Soc. Ital. Biol. Sper. 37 (1961) 766-768.

365. Scassellati-Sforzolini, G. and A. Mariani: Ricerca del 3:4-benzopirene e di altri idrocarburi policiclici nel fumo di sigarette Italiane: Contributo allo studio dell'azione cancerigene del fumo di tobacco [Research on 3:4-benzopyrene and other polycyclic hydrocarbons in the smoke of Italian cigarettes: Contribution to the study of the carcinogenic action of tobacco smoke]; Ricerca Sci. Rend. 1 (2) (1961) 98-117.

366. Scassellati-Sforzolini, G. and G. Saldi: Ulteriori ricerche sugli idrocarburi policiclici del fumeso di sigarette. (Confronto tra il fumo aspirato e quello raccolto nell'aria ambiente) [Further research on the polycyclic hydrocarbon content of the smoke of cigarettes. (Comparison between the aspirated smoke from cigarettes and that recovered from the ambient air)]; Boll. Soc. Ital. Biol. Sper. 37 (1961) 769-771.
367. Scassellati-Sforzolini, G. and G. Salucci: Prime ricerche sulla presenza di idrocarburi cancerigeni nel fumo delle sigarette Italiane [Initial research on the presence of carcinogenic hydrocarbons in the smoke of Italian cigarettes]; Boll. Soc. Ital. Biol. Sper. 34 (1958) 424-426.

368. Schepartz, A.I., A.C. Mottola, W.S. Schlotzhauer, D.W. DeJong, and J.J. Lam: Effect of ozone treatment of tobacco on leaf lipids and smoke PAH: A pilot-plant trial; $34^{\text {th }}$ Tobacco Chemists' Research Conference, Program Booklet and Abstracts, Vol. 34, Paper No. 29, 1980, p. 15; Tob. Sci. 25 (1981) 120-122.

369. Scherbak, M.P., R.L. Rice, and J.E. de Souza: An absolute method for the determination of 3,4-benzpyrene in cigarette smoke; $17^{\text {th }}$ Tobacco Chemists' Research Conference, Program Booklet and Abstracts, Vol. 17, Paper No. 20, 1963, pp. 15-16.

370. Schlotzhauer, W.S. and O.T. Chortyk: Comparison of pyrolytic products from flue-cured tobacco leaf and a reconstituted tobacco sheet; $28^{\text {th }}$ Tobacco Chemists' Research Conference, Program Booklet and Abstracts, Vol. 28, Paper No. 30, 1974, p. 22; Beitr. Tabakforsch. 8 (1975) 84-88.

371. Schlotzhauer, W.S. and I. Schmeltz: 3,5-Xylenol and other products from pyrolysis of sodium acetate; $22^{\text {nd }}$ Tobacco Chemists' Research Conference, Program Booklet and Abstracts, Vol. 22, Paper No. 23, 1968, p. 18; Schmeltz, I. and W.S. Schlotzhauer: 3,5Dimethylphenol and other products from pyrolysis of sodium acetate; Chem. Comm. (1969) 681-682.

372. Schlotzhauer, W.S. and I. Schmeltz: Pyrogenesis of aromatic hydrocarbons present in cigarette smoke. I. Role of the hexane soluble fraction of tobacco; Beitr. Tabakforsch. 4 (1968) 176-181.

373. Schlotzhauer, W.S. and I. Schmeltz:: Pyrogenesis of aromatic hydrocarbons present in cigarette smoke. II. Pyrolysis products of some representative constituents of the hexane soluble fraction of tobacco; Beitr. Tabakforsch. 5 (1969) 5-8.

374. Schlotzhauer, W.S. and I. Schmeltz: Role of the hexane soluble fraction of tobacco in the formation of aromatic hydrocarbons present in tobacco smoke; $23^{\text {rd }}$ Tobacco Chemists' Research Conference, Program Booklet and Abstracts, Vol. 23, Paper No. 28, 1969, p. 20.

375. Schlotzhauer, W.S., I. Schmeltz, and L.C. Donio: Pyrolytic formation of phenols from high molecular weight tobacco leaf constituents; 20 ${ }^{\text {th }}$ Tobacco Chemists' Research Conference, Program Booklet and Abstracts, Vol. 20, Paper No. 28, 1966, pp. 35-37; Schlotzhauer, W.S., I. Schmeltz, and L.C. Hickey: Pyrolytic formation of phenols from some high molecular weight tobacco leaf constituents and nontobacco materials; Tob. Sci. 11 (1967) 31-34.

376. Schlotzhauer, W.S., I. Schmeltz, and S.F. Osman: Evidence for the origin of monoalkenes in cigarette smoke; Chem. and Ind. (London) (1970) 1377-1378.

377. Schlotzhauer, W.S., R.F. Severson, O.T. Chortyk, R.F. Arrendale, and H.C. Higman: Pyrolytic formation of polynuclear aromatic hydrocarbons from petroleum ether extractable constituents of flue-cured tobacco leaf; J. Agr. Food Chem. 24 (1976) 992-997.

378. Schlotzhauer, W.S., R.F. Severson, O.T. Chortyk, and E.M. Snook: Pyrolytic precursors of polynuclear aromatic hydrocarbons in the petroleum ether extract of tobacco; $29^{\text {th }}$ Tobacco Chemists' Research Conference, 
Program Booklet and Abstracts, Vol. 29, Paper No. 43, 1975, p. 30.

379. Schmeltz, I. (Editor): The chemistry of tobacco and tobacco smoke; Plenum Press, New York NY, 1972, pp. 77-97.

380. Schmeltz, I., K.D. Brunnemann, D. Hoffmann, and A. Cornell: On the chemistry of cigar smoke: Comparisons between experimental little and large cigars; $29^{\text {th }}$ Tobacco Chemists' Research Conference, Program Booklet and Abstracts, Vol. 29, Paper No. 41, 1975, p. 29; Beitr. Tabakforsch. 8 (1976) 367-377.

381. Schmeltz, I. and D. Hoffmann: Nitrogen-containing compounds in tobacco and tobacco smoke; Chem. Rev. 77 (1977) 295-311.

382. Schmeltz, I., D. Hoffmann, and J. Tosk: Naphthalenes in tobacco smoke: Analysis and formation; $29^{\text {th }}$ Tobacco Chemists' Research Conference, Program Booklet and Abstracts, Vol. 29, Paper No. 22, 1975, p. 19; Schmeltz, I., J. Tosk, and D. Hoffmann: Formation and determination of naphthalenes in cigarette smoke; Anal. Chem. 48 (1976) 645-650.

383. Schmeltz, I., D. Hoffmann, and E.L. Wynder: Toxic and tumorigenic agents in tobacco smoke: Analytical methods and modes of origin; in: Proc. $8^{\text {th }}$ Ann. Conf. Trace Substances in Environmental Hlth., edited by Hemphill, Columbia, MO (1974) 281-295.

384. Schmeltz, I. and W.S. Schlotzhauer: Benzo[a]pyrene, phenols, and other products from pyrolysis of the cigarette additive, $(d, l)$-menthol; Nature 219 (1968) 370-371.

385. Schmeltz, I., R.L. Stedman, and W.J. Chamberlain: Improved method for the determination of benzo[ $a]$ pyrene in cigarette smoke condensate; Anal. Chem. 36 (1964) 2499-2500.

386. Schmeltz, I., R.L. Stedman, W.J. Chamberlain, and D. Burdick: Composition studies on tobacco. XX. Bases of cigarette smoke; J. Sci. Food. Agr. 15 (1964) 774-781.

387. Schmeltz, I., J. Tosk, J. Hilfrich, N. Hirota, and D. Hoffmann: Bioassays of naphthalene and alkylnaphthalenes for cocarcinogenic activity. Relation to tobacco carcinogenesis; in: Polynuclear aromatic hydrocarbons. Vol. 3, edited by P.W. Jones and R.I. Freudenthal, Raven Press, New York, NY (1978) 47-60.

388. Schmid, E.R., G. Allmaier, G. Bachlechner, K. Varmuza, and H. Klus: Determination of oxygen and/or sulfur containing polycyclic aromatic compounds in cigarette smoke condensate; $8^{\text {th }}$ Internat. Tob. Sci. Cong., Vienna, Austria, 1984, CORESTA Inf. Bull., Spec. Edition 1984: Paper S16, 56; Schmid, E.R., G. Bachlechner, K. Varmuza, and H. Klus: Determination of polycyclic aromatic hydrocarbons, polycyclic aromatic sulfur and oxygen heterocycles in cigarette smoke condensate; Fresenius Z. Anal. Chem. 322 (1985) 213-219.

389. Schoental, R.: Carcinogenic activity of 3,4,9,10dibenzpyrene; Acta Unio Internat. Contra Cancrum 15 (1959) 216-219.

390. Schüller, D., C.J. Drews, and H.P. Harke: Analytische Untersuchungen an Gasphasenkondensat [Analytical examination of gas-phase condensate of cigarette smoke]; Beitr. Tabakforsch. 6 (1971) 84-88.

391. Schumacher, J.N., F.W. Best, and C.R. Green: Smoke composition: A detailed investigation of the watersoluble portion of cigarette smoke; RDR, 1974, No. 7 , September 5, see www.rjrtdocs.com 501003488 -3512;
Schumacher, J.N., C.R. Green, and F.W. Best: The composition of the water-soluble portion of cigarette smoke particulate phase; $29^{\text {th }}$ Tobacco Chemists' Research Conference, Program Booklet and Abstracts, Vol. 29, Paper No. 38, 1975, p. 27; Schumacher, J.N., C.R. Green, F.W. Best, and M.P. Newell: Smoke composition. An extensive investigation of the watersoluble portion of cigarette smoke; J. Agr. Food Chem. 25 (1977) 310-320.

392. Schumacher, J.N., C.R. Green, and F.W. Best: Smoke composition: Homogenized vs unhomogenized tobacco blend; RDR, 1972, No. 5, March 27, see www.rjrtdocs. com 501002566 -2626.

393. Schumacher, J.N., J.J. Murphy, J.M. Conner, and A.L. Angel: Sidestream smoke bases; R\&DM, 1987, No. 54, March 27, see www.rjrtdocs.com 509803520 -3529.

394. Seehofer, F. and D. Hanssen: Die Kapillarpresse, eine Rauchmaschine zur Gewinnung von nativem Rauchkondensat. 2. Mitteilung: Die automatische Kapillarpresse [An automatic capillary press, a smoking machine for collecting instant smoke condensate 2nd report: An automatic capillary press]; Beitr. Tabakforsch. 3 (1965) 291-300.

395. Seelkopf, C.: Über die Isolierung cancerogener Stoffe aus dem Zigarettenteer [On the isolation of carcinogenic compounds from cigarette tar]; Z. Lebensm. Untersuch. Forsch. 100 (1955) 218-222.

395a. Segura, G.: The contribution of cigarette paper to the benzo $[a]$ pyrene in smoke; Memorandum to F.E. Resnik, February 1, 1966, see www.pmdocs.com 1000702895/2901; Resnik, F.E.: Contribution of cigarette paper to benzo[a]pyrene in smoke; Memorandum to H. Wakeham, February 1, 1966, see www.pmdocs.com 1001898064.

396. Severson, R.F., O.T. Chortyk, and W.J. Chamberlain: Gamma radiation effects on cigarettes; Beitr. Tabakforsch. 8 (1975) 136-140.

397. Severson, R.F., W.S. Schlotzhauer, R.F. Arrendale, and E.M. Snook: Correlation of polynuclear aromatic hydrocarbon formation between pyrolysis and smoking; $29^{\text {th }}$ Tobacco Chemists' Research Conference, Program Booklet and Abstracts, Vol. 29, Paper No. 44, 1975, p. 30; Beitr. Tabakforsch. Int. 9 (1977) 23-37.

398. Severson, R.F., W.S. Schlotzhauer, O.T. Chortyk, R.F. Arrendale, and E.M. Snook, Precursors of polynuclear aromatic hydrocarbons in tobacco smoke; in: $3^{\text {rd }}$ International Symposium on Carcinogenesis and Mutagenesis, edited by P.W. Jones and P. Leber, Ann Arbor Science, Ann Arbor MI, 1979, pp. 277-298.

399. Severson, R.F., E.M. Snook, R.F. Arrendale, and O.T. Chortyk: Comparison of levels of polynuclear aromatic hydrocarbons in the smoke of different cigarettes; 29 $9^{\text {th }}$ Tobacco Chemists' Research Conference, Program Booklet and Abstracts, Vol. 29, Paper No. 19, 1975, p. 18.

400. Severson, R.F., E.M. Snook, R.F. Arrendale, and O.T. Chortyk: Gas chromatographic quantitation of polynuclear aromatic hydrocarbons in tobacco smoke; Anal. Chem. 48 (1976) 1866-1872.

401. Severson, R.F., E.M. Snook, W.J. Chamberlain, and O.T. Chortyk: A chromatographic analysis of polynuclear aromatic hydrocarbons in small quantities of cigarette smoke condensate; $28^{\text {th }}$ Tobacco Chemists' Research Conference, Program Booklet and Abstracts, Vol. 28, Paper No. 39, 1974, p. 27; Beitr. Tabakforsch. 
8 (1976) 273-282.

402. Severson, R.F., E.M. Snook, H.C. Higman, O.T. Chortyk, and F.J. Akin: Isolation, identification, and quantitation of the polynuclear aromatic hydrocarbons in tobacco smoke; Carcinogenesis - Comp. Survey 1 (1976) 253-270.

403. Shamberger, R.J.: Reduced benzo[a]pyrene and phenolic content from experimental cigarettes; Nature 211 (1966) 86.

404. Shear, M.J. and J. Leiter: Studies in carcinogenesis. XVI. Production of subcutaneous tumors in mice by miscellaneous polycyclic compounds; J. Natl. Cancer Inst. 2 (1941) 241-258.

405. Shelar, G.R.: The effect of cigarette lighting technique on the benzo $[a]$ pyrene in mainstream smoke; RDM, 1977, No. 6, February 10, see www.rjrtdocs.com $500617080-7083$.

406. Shelar, G.R., T.R. Conner, and D.A. Colby: Analysis of benzo[ $a]$ pyrene in cigarette smoke by highperformance liquid chromatography; R\&DM, 1981, No. 40, October 20, see www.rjrtdocs.com 500609747 $-9759$.

407. Shieh, S.F., B.H. Song, and D.L. Davis: Pyrolytic formation of some polynuclear aromatic hydrocarbons from the ionones. $33^{\text {rd }}$ Tobacco Chemists' Research Conference, Program Booklet and Abstracts, Vol. 33, Paper No. 52, 1979, p. 28.

408. Shubik, P. and J.L. Hartwell: Survey of compounds which have been tested for carcinogenic activity, Suppl. 1; USPHS Publ. No. 149 (1957) Washington, DC; Suppl. 2; USPHS Publ. No. 149 (1969) Washington, DC.

409. Slaga, T.J. and R.K. Boutwell: Inhibition of the tumorinitiating ability of the potent carcinogen 7,12dimethylbenz $[a]$ anthracene by the weak tumor initiator 1,2,3,4-dibenzanthracene; Cancer Res. 37 (1977) $129-133$.

410. Slaga, T.J. and J. DiGiovanni: Inhibition of chemical carcinogenesis; in: Chemical carcinogens, Second edition, edited by C.E. Searle, American Chemical Society Monograph 182, American Chemical Society, Washington, DC, 1984, pp. 1279-1321.

411. Slaga, T.J., L. Jecker, W.M. Bracken, and C.E. Weeks: The effects of weak or non-carcinogenic polycyclic hydrocarbons on 7,12-dimethylbenz $[a]$ anthracene and benzo[a]pyrene; Cancer Lett. 7 (1979) 51-59.

412. Slaga, T.J., A. Viaje, S.G. Buty, and W.M. Bracken: Dibenz $[a, c]$ anthracene: A potent inhibitor of skintumor initiation by 7,12-dimethylbenz $[a]$ anthracene; Res. Comm. Chem. Pathol. Pharmacol. 19 (1978) 477-483.

413. Smith, C.J., T.A. Perfetti, M.J. Morton, A. Rodgman, R. Garg, C.D. Selassie, and C. Hansch: The relative toxicity of substituted phenols reported in cigarette mainstream smoke; 2002 CORESTA Congress, New Orleans, LA, September, Paper ST 9; Toxicol. Sci. 69 (2002) 265-278.

414. Smith, C.J., T.A. Perfetti, M.A. Rumple, A. Rodgman, and D.J. Doolittle: An international literature survey of IARC carcinogens in cigarette mainstream smoke; $53^{\text {rd }}$ Tobacco Science Research Conference, Program Booklet and Abstracts, Vol. 53, Paper No. 28, 1999, pp. 34-35; "IARC Group 2A carcinogens" reported in cigarette mainstream smoke; Food Chem. Toxicol. 39 (2000) 371-383. 414a. Smith, C.J., T.A. Perfetti, M.A. Rumple, A. Rodgman, and D.J. Doolittle: "IARC Group 2B Carcinogens" reported in cigarette mainstream smoke; Food Chem. Toxicol. 38 (2000) 825-848; Food Chem. Toxicol. 39 (2001) 183-205. [correction of publication (Food Chem. Toxicol. 38 (2000) 825-848)].

415. Smith, W.T. Jr, C.Y. Shiue, and J.M. Patterson: Pyrolysis of sulfur amino acids; $24^{\text {th }}$ Tobacco Chemists' Research Conference, Program Booklet and Abstracts, Vol. 24, Paper No. 17, 1970, p. 11.

416. Snook, M.E.: Gel filtration of methyl-substituted polynuclear aromatic hydrocarbons; Anal. Chim. Acta 81 (1976) 423-427.

417. Snook, M.E. and O.T. Chortyk: Advances in lipophilic gel chromatography of tobacco and tobacco smoke components; Recent Adv. Tob. Sci. 12 (1986) 237-297.

418. Snook, M.E., P.J. Fortson, L.B. Smith, and O.T. Chortyk: Isolation and identification of nitrogen analogs of polynuclear aromatic hydrocarbons; $30^{\text {th }}$ Tobacco Science. Research Conference, Program Booklet and Abstracts, Vol. 30, Paper No. 47, 1976, p. 32; Isolation and identification of aza-arenes of tobacco smoke; $32^{\text {nd }}$ Tobacco Science. Research Conference, Program Booklet and Abstracts, Vol. 32, Paper No. 46, 1978, p. 25; Snook, M.E., P.J. Fortson, and O.T. Chortyk: Isolation and identification of aza-arenes of tobacco smoke; Beitr. Tabakforsch. Int. 11 (1981) 67-78.

419. Snook, M.E., R.F. Severson, R.F. Arrendale, H.C. Higman, and O.T. Chortyk:: High molecular weight polynuclear aromatic hydrocarbons of cigarette smoke; $29^{\text {th }}$ Tobacco Chemists' Research Conference, Program Booklet and Abstracts, Vol. 29, Paper No. 21, 1975, p. 19; The identification of high molecular weight polynuclear aromatic hydrocarbons in a biologically active fraction of cigarette smoke condensate; Beitr. Tabakforsch. Int. 9 (1977) 79-101.

420. Snook, M.E., R.F. Severson, R.F. Arrendale, H.C. Higman, and O.T. Chortyk: Multi-alkylated polynuclear aromatic hydrocarbons of tobacco smoke: Separation and identification; Beitr. Tabakforsch. Int. 9 (1978) 222-247.

421. Snook, M.E., R.F. Severson, H.C. Higman, R.F. Arrendale, and O.T. Chortyk: Polynuclear aromatic hydrocarbons of tobacco smoke: Isolation and identification; Beitr. Tabakforsch. 8 (1976) 250-272.

422. Snook, M.E., R.F. Severson, H.C. Higman, and O.T. Chortyk: Isolation and identification of polynuclear aromatic hydrocarbons of tobacco smoke; $28^{\text {th }}$ Tobacco Chemists' Research Conference, Program Booklet and Abstracts, Vol. 28, Paper No. 40, 1974, p. 27.

423. Stamey, T.W. Jr and J.T. Dobbins Jr: Fluorometric determination of polynuclear hydrocarbons; RDR, 1965, No. 45, October 11, see www.rjrtdocs.com $500966272-6303$

424. Stamey, T.W. Jr, R.B. Hege Jr, F.A. Thacker Jr, and J.T. Dobbins Jr: Fluorometric method for determination of polynuclear hydrocarbons in cigarette smoke; RDR, 1971, No. 12, May 18, see www.rjrtdocs.com 501002076 -2104; A rapid thin-layer method for fluorometric determination of benzo $[a]$ pyrene in cigarette smoke; RDR, 1971, No. 20, September 16, see www.rjitdocs.com 504300953 -0953.

425. Stedman, R.L: The chemical composition of tobacco 
and tobacco smoke; Chem. Rev. 68 (1968) 153-207.

426. Steiner, P.E. and H.L. Falk: Summation and inhibition effects of weak and strong carcinogenic hydrocarbons, 1:2-benzanthracene, chrysene, 1:2:5:6-dibenzanthracene, and 20-methylcholanthrene; Cancer Res. 11 (1951) 56-63.

427. Stowe, M.E. and J.L. Harris: A rapid gas chromatographic analysis for fluoranthene and pyrene found in cigarette smoke; RDR, 1970, No. 47, December 3, see www.rjirtdocs.com $500510348-0353$.

428. Sula, J.P.: Exogenous carcinogens in the human organism; Acta Unio Internat. Contra Cancrum 15 (1959) 688-691.

429. Sula, J.P.: The carcinogen 3,4-benzpyrene in the living environment and human organism; Neoplasma 10 (1963) 571-579.

430. Swain, A.P., J.E. Cooper, R.L. Stedman, and F.G. Bock: Composition studies on tobacco. XL. Large scale fractionation of the neutrals of cigarette smoke condensate using adsorption chromatography and solvent partitioning; $23^{\text {rd }}$ Tobacco Chemists' Research Conference, Program Booklet and Abstracts, Vol. 23, Paper No. 14, 1969, p. 9; Beitr. Tabakforsch. 5 (1969) 109-114.

431. Swauger, J.E., M.J. Morton, C.A. Rahn, B.B. Collie, E.A. Bombick, J.T. Avalos, M.F. Borgerding, and D.C. Rees: A comparison of the mainstream cigarette smoke chemistry and mutagenicity of a representative sample of the US cigarette market with Kentucky Reference Cigarettes K1R4F and K1R5F; The Toxicologist 36 (1) Part 2 (1997) 152 Abstract 774. Presented at the Society of Toxicology Annual Meeting (March 1997), see www.rjitdocs.com $525770805-0819$.

432. Szent-Gyorgyi, A.: Removal of polycyclic aromatic hydrocarbons from cigarette mainstream smoke by chloranil; Personal communication in 1960 to R.J. Reynolds Tobacco Company, see Rodgman, A. and L.C. Cook: The analysis of cigarette smoke condensate. XVII. Chloranil and 2,4,7-trintrofluorenone as filter-tip additives; RDR, 1960, No. 38, December 7, see www.rjrtdocs.com $501008695-8704$.

433. Takayama, S. and K. Oota: Chemical analysis of cigarette tar produced by human smoking; Gann 51 (1960) 97-103.

434. Tennessee Eastman Corporation: Composition of tobacco smoke; Tennessee Eastman Corp. Res. Rpt. No. 4-1201-1 (August 21, 1956).

435. Tennessee Eastman Corporation: Composition of tobacco smoke. II. Composition of tobacco extract and its pyrolysis products; Tennessee Eastman Corp. Res. Rpt. No. 4-1201-2 (April 13, 1959).

436. Tennessee Eastman Corporation: Composition of tobacco smoke. III. Effects of the extraction of tobacco on amount of benzo[a]pyrene in cigarette smoke tar; Tennessee Eastman Corp. Res. Rpt. NO. 4-1201-3 (July 14, 1959).

437. Testa, A., P. Testa, and J.L. Cuzin: A rapid analytical technique for routine determination of benzo $[a]$ pyrene in cigarette smoke condensate; $17^{\text {th }}$ Tobacco Chemists' Research Conference, Program Booklet and Abstracts, Vol. 17, Paper No. 19, 1963, p. 15.

438. Tomita, H. and D. Yoshida: Pyrolytic formation of benzo $[a]$ pyrene from various compounds; $29^{\text {th }}$ Tobacco Chemists' Research Conference, Program Booklet and Abstracts, Vol. 29, Paper No. 45, 1975, p. 31.
439. Tricker, A.R., G. Scherer, and F. Adlkofer: Influence of tobacco nitrate content on the yields of selected mainstream smoke components; $47^{\text {th }}$ Tobacco Chemists' Research Conference, Program Booklet and Abstracts, Vol. 47, Paper No. 40, 1993, p. 46.

440. Trosko, J.E. and B.L. Upham: The emperor wears no clothes in the field of carcinogen risk assessment: Ignored concepts in cancer risk assessment; Mutagenesis 20 (2005) 81-92.

441. Tso, T.C. and J.F. Chaplin: Simple correlation and multiple regression among leaf characteristics, smoke components, and biological responses of bright tobaccos; USDA Tech. Bull. 1551 (1977) 1-135.

442. Tso, T.C., J.F. Chaplin, J.D. Adams, and D. Hoffmann: Simple correlation and multiple regression among leaf and smoke characteristics of burley tobaccos; $7^{\text {th }}$ Internat. Tob. Sci. Cong., Manila, The Philippines, CORESTA Inf. Bull., Spec. Edition 1980: Paper APST 05, 137; Beitr. Tabakforsch. Int. 11 (1982) 141-150.

443. Tso, T.C., G. Rathkamp, and D. Hoffmann Chemical studies on tobacco smoke. XXI. Correlation and multiple regression among selected cigarette-smoke constituents and leaf characteristics of bright tobacco; Beitr. Tabakforsch. 7 (1973) 190-194.

444. Uhrig, M.S., E.L. White, B.M. Gordon, M.F. Borgerding, R.D. Hicks, and E.J. Nanni: Quantitation of selected components in mainstream smoke particulate phase (MSPP) of a Kentucky Reference Cigarette $(1 \mathrm{R} 4 \mathrm{~F})$ and a cigarette that heats rather than burns tobacco; 42 ${ }^{\text {nd }}$ Tobacco Chemists' Research Conference, Program Booklet and Abstracts, Vol. 47, Paper No. 57, 1988, p. 45.

445. USPHS: Smoking and health: Report of the Advisory Committee to the Surgeon General of the Public Health Service; PHS Publ. No. 1103 (1964) 47-65.

446. USPHS: Smoking and health: Report of the Advisory Committee to the Surgeon General of the Public Health Service; PHS Publ. No. 1103 (1964) 57.

447. USPHS: Smoking and health. Report of the Advisory Committee to the Surgeon General of the Public Health Service; DHEW Publ. No. (PHS) 1103 (1964) 58.

448. USPHS: Smoking and health. A report of the Surgeon General; DHEW Publ. No. (PHS) 79-50066 (1979).

449. USPHS: Smoking and health. A report of the Surgeon General; DHEW Publ. No. (PHS) 79-50066 (1979) 14 51-52.

450. USPHS: Smoking and health. A report of the Surgeon General; DHEW Publ. No. (PHS) 79-50066 (1979) 14 51-52, 54 (Table 15).

451. USPHS: The health consequences of smoking. The changing cigarette. A report of the Surgeon General; DHHS Publ. No. (PHS) 81-50156 (1981) 36.

452. USPHS: The health consequences of smoking. Cancer. A report of the Surgeon General; DHHS Publ. No. (PHS) 82-50179 (1982).

453. USPHS: The health consequences of smoking. Cancer. A report of the Surgeon General; DHHS Publ. No. (PHS) 82-50179 (1982) 183-235.

454. Van Duuren, B.L.: The polynuclear hydrocarbons in cigarette smoke condensate; Proc. $1^{\text {st }}$ Workshop Conf. on Lung Cancer Research (1958) 52-55.

455. Van Duuren, B.L.: Identification of some polynuclear aromatic hydrocarbons in cigarette-smoke condensate; J. Natl. Cancer Inst. 21 (1958) 1-16.

456. Van Duuren, B.L.: The polynuclear aromatic hydro- 
carbons in cigarette-smoke condensate. II; J. Natl. Cancer Inst. 21 (1958) 623-630.

457. Van Duuren, B.L.: Some aspects of the chemistry of tobacco smoke; Chapter 3 in: Tobacco and health, edited by G. James and T. Rosenthal, Charles C Thomas, Springfield IL, 1962, pp. 33-47.

458. Van Duuren, B.L.: The metabolism of dibenz $[a, h]$ anthracene; Acta Unio Internat. Contra Cancrum 19 (1963) 524-527.

459. Van Duuren, B.L. and N. Nelson: The polycyclic aromatic hydrocarbons in cigarette smoke; Proc. Am. Assoc. Cancer Res. 2 (4) (1958) 353.

460. Van Duuren, B.L., A. Sivak, L. Langseth, B.M. Goldschmidt, and A. Segal: Initiators and promoters in tobacco carcinogenesis; in: Toward a less harmful cigarette, edited by E.L. Wynder and D. Hoffmann, Natl. Cancer Inst. Monograph 28 (1968) 173-180.

461. Van Duuren, B.L., A. Sivak, A. Segal, L. Orris, and L. Langseth: The tumor-promoting agents of tobacco leaf and tobacco smoke condensate; J. Natl. Cancer Inst. 37 (1966) 519-526.

462. Wahl, R.: Carbohydrate derivatives in tobacco and in smoke; Tabakforschung 4(19) (1957) 42; Tabakforschung 4(22) (1957) 61-64.

463. Wahlberg, I., A.-M. Eklund, C. Vogt, C.R. Enzell, and J.-E. Berg: Tobacco chemistry. 65. Two new 7,8epoxycembranoids from tobacco; Acta Chem. Scand. B40 (1986) 855-860.

464. Wahlberg, I. and C.R. Enzell: Tobacco isoprenoids. [A review of literature between 1975 and 1984] Nat. Prod. Rpt. 4 (3) (1987) 237-76.

465. Wakeham, H.: Recent trends in tobacco and tobacco smoke research; Symposium on the Composition of Tobacco and Tobacco Smoke, Am. Chem. Soc. Mtg., Washington, DC (1971); in: The chemistry of tobacco and tobacco smoke, edited by I. Schmeltz, Plenum Press, New York, NY, 1972, pp. 1-20.

466. Waldman, J.M., P.J. Lioy, A. Greenberg, and P. Butler: Analysis of human exposure to benzo(a)pyrene via inhalation and food ingestion in the total human exposure study (THEES); J. Exposure Anal. Environ. Epidemiol. 1 (1991) 193-225.

467. Walker, W.E. Jr: Phosphorimetric determination of polynuclear aromatic hydrocarbons; RDR, 1961, No. 6, January 27, see www.rjrtdocs.com 500936069 -6096.

468. Walters, D.B., W.J. Chamberlain, E.M. Snook, and O.T. Chortyk: High-pressure liquid chromatography for monitoring benzo $[a]$ pyrene contents of cigarette smoke condensate fractions; Anal. Chim. Acta 73 (1974) 194-197.

469. Waltz, P. and M. Häusermann: Sur un traitement du tabac en vue de diminuer la teneur en hydrocarbures policycliques de la fumée de cigarettes [On a treatment of tobacco in order to reduce the content of polycyclic hydrocarbons in the smoke from cigarettes]; $\mathrm{Z}$. Präventivmed. 8 (1963) 111-124.

470. Weber, K.H.: Recent changes in tobacco products and their acceptance by the consumer; Proceedings 6th International Tobacco Scientific Congress, Tokyo, Japan (1976) 47-63; United States Public Health Service: Smoking and health. A report of the Surgeon General; DHEW Publ. No. (PHS) 79-50066 (1979): See Chapter 14, pp. 111-112.

470a. Weil-Malherbe, H.: [Formation of water-soluble complexes of PAHs with purines]; Biochem. J. 40
(1946) 351, 363.

471. Whitehead, J.K.: Some chemical aspects of smoking and health; Chem. Soc. Mtg., London, England, see abstr. in Chem. and Ind. (London) (1965) 1222-1223.

472. Whitehead, J.K. and P. Dickens: A study of carcinogenic components of cigarette smoke; Brit. Emp. Cancer Camp., $41^{\text {st }}$ Ann. Rpt., Pt. II (1963) 26-27.

473. Wieske, R.: Untersuchungen über cancerogene Bestandteile im Tabakrauch [Study of the carcinogenic components in tobacco smoke]; Arzneimittelforsch. 7 (1957) 324-329.

474. Williams, R., C. Sparacino, B. Petersen, J. Bumgarner, R.H. Jungers, and J. Lewtas: Comparative characterization of organic emissions from diesel particles, coke oven mains, roofing tar vapors and cigarette smoke condensate; Internat. J. Environ. Anal. Chem. 26 (1986) 27-49.

475. Williams, R.W. and Science Information Division, R\&D, RJRT: Constituents reported in tobacco and tobacco smoke; April 15, 1997, pp. 1-134.

475a. World Health Organization: Advancing knowledge regulating tobacco products; Monograph (2000) 1-120.

476. Wright, G.F: Studies with tobacco smoke condensate; in: Proc. 3rd Natl. Cancer Conf., June, 1956, JB Lippincott Company, 1957, pp. 479-484.

477. Wright, G.F: Personal communication; see Rodgman, A.: RDM, 1957, No. 25, July 22, www.rjrtdocs.com $500610651-0666$.

478. Wright, G.F and E.L. Wynder: Fractionation of cigarette tar; Proc. Am. Assoc. Cancer Res. 2 (1) (1955) 55.

479. Wright, G.F and E.L. Wynder: Further chemical studies of cigarette smoke condensate; Proc. Am. Assoc. Cancer Res. 2 (2) (1956) 159.

480. Wynder, E.L. (Editor): The biologic effects of tobacco: With special emphasis on the clinical and experimental aspects; Little, Brown \& Co., Boston MA, 1955.

481. Wynder, E.L.: Human and experimental relation of tobacco and cancer; Tob. Symp., Long Island Subsection, Am. Chem. Soc. (1956), see Rodgman, A.: RDM, 1956, No. 9, March 16, www.rjrtdocs.com $501009747-9754$.

482. Wynder, E.L.: Environmental causes of cancer in man; Med. Clin. N. America (1956) 629-645.

483. Wynder, E.L.: Statement on the lung cancer-cigarette smoking controversy: Chemical fractionation of cigarette smoke; in: False and misleading advertising (Filter-tip cigarettes), Hearing before Subcommittee of the Committee on Government Operations, House of Representatives, $85^{\text {th }}$ Congress, $1^{\text {st }}$ Session, 1957, pp. 63-114.

484. Wynder, E.L.: Laboratory contributions to the tobaccocancer problem; Symposium on Chemical and biological problems related to smoking, Stockholm, Sweden, 1960; Acta Med. Scand. Suppl. 369 (1961) 63-101.

485. Wynder, E.L.: Studies in tobacco carcinogenesis; Proc. Am. Assoc. Cancer Res. 5 (1) (1964) 70, see Rodgman, A.: RDM, 1964, No. 52, May 5, www.rjrtdocs.com $504913254-3275$.

486. Wynder, E.L., L. Fritz, and N. Furth: Effect of concentration of benzopyrene in skin carcinogenesis; $\mathrm{J}$. Natl. Cancer Inst. 19 (1957) 361-370.

487. Wynder, E.L. and E.A. Graham: Tobacco smoking as a possible etiologic factor in bronchiogenic carcinoma: 
A study of six hundred and eighty-four proved cases; J. Am. Med. Assoc. 143 (1950) 329-336; Doll, R. and A.B. Hill: Smoking and carcinoma of the lung. Preliminary report; Brit. Med. J. 1950 (ii) 739-748; Schrek, R., L.A. Baker, G.P. Ballard, and S. Dolgoff: Tobacco smoking as an etiologic factor in disease; Cancer Res. 10 (1950) 49-58; Doll, R. and A.B. Hill: A study of the aetiology of carcinoma of the lung; Brit. Med. J. 1952 (ii) 1271-1286.

488. Wynder, E.L., E.A. Graham, and A.B. Croninger: Study on the experimental production of cancer with tobacco tar; Proc. Am. Assoc. Cancer Res. 1 (1953) 62-63; Experimental production of carcinoma with cigarette tar; Cancer Res. 13 (1953) 855-864.

489. Wynder, E.L. and S.S. Hecht: Lung cancer; UICC Tech. Rept. Series 25 (1976) 138.

490. Wynder, E.L. and D. Hoffmann: The role of higher polycyclic hydrocarbons in tobacco carcinogenesis; Proc. Am. Assoc. Cancer Res. 3 (1) (1959) 74, see Rodgman, A.: RDM, 1959, No. 41, April 20, www.rjrtdocs.com 501009779 -99792; A study of tobacco carcinogenesis. VII. The role of higher polycyclic hydrocarbons; Cancer 12 (1959) 1079-1086.

491. Wynder, E.L. and D. Hoffmann: The carcinogenicity of benzofluoranthenes; Cancer 12 (1959) 1194-1199.

492. Wynder, E.L. and D. Hoffmann: Studies in tobacco carcinogenesis; Proc. Am. Assoc. Cancer Res. 3 (2) (1960) 164, see Rodgman, A.: RDM, 1960, No. 32, April 13, www.rjrtdocs.com 502816003 -6012.

493. Wynder, E.L. and D. Hoffmann: Some practical aspects of the smoking-cancer problem; New Eng. J. Med. 262 (1960) 540-545.

494. Wynder, E.L. and D. Hoffmann: Biological and chemical studies of tobacco smoke condensate; Proc. Am. Assoc. Cancer Res. 3 (3) (1961) 280, see Rodgman, A.: RDM, 1961, No. 35, April 20, www.rjrtdocs.com $504913207-3220$.

495. Wynder, E.L. and D. Hoffmann: Present status of laboratory studies on tobacco carcinogenesis; Acta Pathol. Microbiol. Scand. 52 (1961) 119-132.

496. Wynder, E.L. and D. Hoffmann: A study of air pollution carcinogenesis. III. Carcinogenic activity of gasoline engine exhaust condensate; Cancer 15 (1962) 103-108.

497. Wynder, E.L. and D. Hoffmann: Bioassays on the carcinogenicity of tobacco smoke condensate and air pollutants; Proc. Am. Assoc. Cancer Res. 4 (1) (1963) 73, see Rodgman, A.: RDM, 1963, No. 44, June 7, www.rjrtdocs.com 504913230 -3249.

498. Wynder, E.L. and D. Hoffmann: Ein experimenteller Beitrag zur Tabakrauchkanzerogenese [An experimental contribution to tobacco smoke carcinogenesis]; Deut. Med. Wchnschr. 88 (1963) 623-628.

499. Wynder, E.L. and D. Hoffmann: Unpublished 1963 results described in: Tobacco and tobacco smoke: Studies in experimental carcinogenesis; Wynder, E.L. and D. Hoffmann, Academic Press, New York, NY, 1967, pp. 234-236.

500. Wynder, E.L. and D. Hoffmann: Experimental tobacco carcinogenesis; Adv. Cancer Res. 8 (1964) 249-453.

501. Wynder, E.L. and D. Hoffmann: Experimental tobacco carcinogenesis; Adv. Cancer Res. 8 (1964) 249-453, see pp. 294-295.

502. Wynder, E.L. and D. Hoffmann: Experimental tobacco carcinogenesis; Adv. Cancer Res 8 (1964) 249-453, see p. 315.

503. Wynder, E.L. and D. Hoffmann: Experimental tobacco carcinogenesis; Adv. Cancer Res. 8 (1964) 249-453, see pp. 316-317.

504. Wynder, E.L. and D. Hoffmann: Experimental tobacco carcinogenesis; Adv. Cancer Res. 8 (1964) 249-453, see p. 318

505. Wynder, E.L. and D. Hoffmann: Experimental tobacco carcinogenesis; Adv. Cancer Res. 8 (1964) 249-453, see pp. 323-326.

506. Wynder, E.L. and D. Hoffmann: Experimental tobacco carcinogenesis; Adv. Cancer Res. 8 (1964) 249-453, see p. 378.

507. Wynder, E.L. and D. Hoffmann: Reduction of tumorigenicity of cigarette smoke. An experimental approach; J. Am. Med. Assoc. 192 (1965) 88-94.

508. Wynder, E.L. and D. Hoffmann: Tobacco and tobacco smoke: Studies in experimental carcinogenesis; Academic Press, New York, NY, 1967.

509. Wynder, E.L. and D. Hoffmann: Tobacco and tobacco smoke: Studies in experimental carcinogenesis; Academic Press, New York NY, 1967, see p. 246.

510. Wynder, E.L. and D. Hoffmann: Tobacco and tobacco smoke: Studies in experimental carcinogenesis; Academic Press, New York, NY, 1967, see p. 334.

511. Wynder, E.L. and D. Hoffmann: Tobacco and tobacco smoke: Studies in experimental carcinogenesis; Academic Press, New York, NY, 1967, see pp. 517-518

512. Wynder, E.L. and D. Hoffmann: Tobacco and tobacco smoke: Studies in experimental carcinogenesis; Academic Press, New York, NY, 1967, see p. 532.

513. Wynder, E.L. and D. Hoffmann: Tobacco and tobacco smoke: Studies in experimental carcinogenesis; Academic Press, New York, NY, 1967, see pp. 625-626.

514. Wynder, E.L. and D. Hoffmann: Tobacco and tobacco smoke: Studies in experimental carcinogenesis; Academic Press, New York, NY, 1967, see p. 636.

514a. Wynder, E.L. and D. Hoffmann: Selected laboratory methods in tobacco carcinogenesis; in: Methods in cancer research, Vol. 4, edited by H. Busch, Academic Press, New York, NY, 1968, pp. 3-52.

514b. Wynder, E.L. and D. Hoffmann (Editors): Toward a less harmful cigarette; Natl. Cancer Inst. Monograph 28, 1968

515. Wynder, E.L. and D. Hoffmann: Experimental tobacco carcinogenesis; Science 162 (1968) 862-871.

515a. Wynder, E.L. and D. Hoffmann: The epidermis and the respiratory tract as bioassays systems in tobacco carcinogenesis; Brit. J. Cancer 24 (1970) 574-587.

516. Wynder, E.L., P. Kopf, and H. Ziegler: Dose response with cigarette tar; Proc. Am. Assoc. Cancer Res. 2 (3) (1957) 261, see Rodgman, A.: RDM, 1961, No. 35, April 20, see www.rjrtdocs.com 504913207 -3220; A study of tobacco carcinogenesis. II. Dose response studies; Cancer 10 (1957) 1193-1200.

517. Wynder, E.L. and J. Mann: A study of tobacco carcinogenesis. III. Filtered cigarettes; Cancer 10 (1957) 1201-1205.

518. Wynder, E.L. and G.F Wright: Fractionation of cigarette tar; Proc. Am. Assoc. Cancer Res. 2 (1) (1955) 55.

519. Wynder, E.L. and G.F Wright: Studies on the 
identification of carcinogens in cigarette tar; Proc. Am. Assoc. Cancer Res. 2 (2) (1956) 159; A study of tobacco carcinogenesis. I. The primary fractions; Cancer 10 (1957) 255-271.

520. Wynder, E.L., G.F Wright, and J. Lam: A study of tobacco carcinogenesis. V. The role of pyrolysis; Proc. Am. Assoc. Cancer Res. 2 (4) (1958) 357-358; see Rodgman, A.: RDM, 1958, No. 38, April 25, www.rjrtdocs.com 501009759-9774; Cancer 11 (1958) 1140-1148.

521. Wynder, E.L., G.F Wright, and J. Lam: A study of tobacco carcinogenesis. VI. The role of precursors; Cancer 12 (1959) 1073-1078.

522. Yamagiwa, K. and K. Ichikawa: Experimentelle Studie über die Pathogenese der Epithelial-geschwülste. I [Experimental study of the pathogenesis of epithelial tumors. I.]; Tokyo Igakkai Zassi 15 (1915) 295-344; II; Tokyo Igakkai Zassi 17 (1917) 19-67; III; Tokyo Igakkai Zassi 19 (1918) 483-495; Yamagiwa, K.: Collected papers on artificial production of cancer; Maruzen Company Ltd., Tokyo, Japan, 1965.

523. Yamamoto, T., Y. Suga, C. Tokura, T. Toda, and T. Okada: Effect of cigarette circumference on formation rates of various components in mainstream smoke; Beitr. Tabakforsch. Int. 13 (1985) 81-87.

524. Zane, A.: Determination of anthracene and pyrene in cigarette smoke by gas chromatography; Tob. Sci. 12 (1968) 54-57.

525. Zane, A.: Determination of phenanthrene in cigarette smoke by gas chromatography; Tob. Sci. 12 (1968) 77-80.

526. Zapior, B., J. Platek, and J. Kaleta: Polycyclic aromatic hydrocarbons in the smoke of domestic Polish cigarette brands; Roczniki Chemii 33 (1959) 243-245.

527. Zha, Q., E.M. Reddick, and S.C. Moldoveanu: Analysis of polycyclic aromatic hydrocarbons in sidestream cigarette smoke using a GC/MS technique; $56^{\text {th }}$ Tobacco Science Research Conference, Lexington KY, Program Booklet and Abstracts, Vol. 56, Paper No. 62, 2002, pp. 57-58.
528. Zhang, C., B. Wang, and J. Shi: Determination of benzo $[a]$ pyrene in cigarette smoke total particulate matter by two-dimensional chromatography; 2002 CORESTA Congress, Paper ST 28.

529. Zhang, L., K. Sannes, A.J. Shusterman, and C. Hansch: The structure-activity relationship of skin carcinogenicity of aromatic hydrocarbons and heterocycles; Chem.-Biol. Interactions 81 (1992) 149-180.

530. Zhang, Y.: Studies of aromatic hydrocarbon carcinogenicity; Master of Science Thesis, Univ. of Texas El Paso, December, 1996, pp. 1-125.

Addresses for correspondence:

Alan Rodgman

2828 Birchwood Drive

Winston-Salem, North Carolina, 27103-3410, USA

E-mail: arodgman@triad.rr.com

Thomas A. Perfetti

Perfetti and Perfetti, LLC

2116 New Castle Drive

Winston-Salem, North Carolina, 27103-5750, USA

E-mail:tperfetti@triad.rr.com 